\title{
Nonlinear Dynamic Analysis of High-Voltage Overhead Transmission Lines
}

\author{
Meng Zhang $\mathbb{D}^{1},{ }^{1}$ Guifeng Zhao $\mathbb{D}^{1},{ }^{1}$ and Jie Li $\mathbb{C D}^{2,3}$ \\ ${ }^{1}$ School of Civil Engineering, Zhengzhou University, Zhengzhou 450001, China \\ ${ }^{2}$ College of Civil Engineering, Tongji University, Shanghai 200092, China \\ ${ }^{3}$ State Key Laboratory for Disaster Reduction in Civil Engineering, Tongji University, Shanghai 200092, China \\ Correspondence should be addressed to Guifeng Zhao; gfzhao@zzu.edu.cn
}

Received 24 July 2017; Revised 15 February 2018; Accepted 8 March 2018; Published 19 April 2018

Academic Editor: Songye Zhu

Copyright (C) 2018 Meng Zhang et al. This is an open access article distributed under the Creative Commons Attribution License, which permits unrestricted use, distribution, and reproduction in any medium, provided the original work is properly cited.

\begin{abstract}
According to a generalized Hamilton's principle, three-dimensional (3D) nonlinear vibration equations for overhead transmission lines that consider geometric nonlinearity are established. Based on the characteristics of an actual transmission line, the 3D equations are simplified to two-dimensional equations, and the nonlinear vibration behavior of transmission lines is investigated by combining theoretical analysis with numerical simulation. The results show that transmission lines have inherently nonlinear vibration characteristics. When in free vibration, a transmission line can undergo nonlinear internal resonance, even when its initial out-of-plane energy is relatively low; as its initial out-of-plane energy increases, the coupling of in-plane and out-of-plane vibration becomes stronger. When forced to vibrate by an external excitation, due to the combined action of internal and primary resonance, the vibration energy of a transmission line transfers from the out-of-plane direction to the in-plane direction that is not directly under the excitation, resulting in an increase in the dynamic tension and the displacement amplitude of the transmission line. Increasing damping can consume the vibration energy of a transmission line but cannot prevent the occurrence of internal resonance.
\end{abstract}

\section{Introduction}

High-voltage overhead transmission lines (HVOTLs) are important carriers within power systems, and their safety directly affects the normal operation of the whole power system. Currently, aluminum conductor steel-reinforced (ACSR) cables are the most widely used transmission lines in engineering applications. ACSR cables, characterized by their light weight, small damping, and long spans, can only bear axial tension. Generally, the vibration of a transmission line in the plane where the transmission line is located under the action of gravity is referred to as in-plane vibration, and the vibration in the plane vertical to the aforementioned plane is referred to as out-of-plane vibration. A transmission line is often considered as a single-cable structure in calculation. According to the suspended cable theory [1], the in-plane stiffness of a transmission line is a result of its internal force, and the out-of-plane stiffness of a transmission line is relatively low. Therefore, the vibration of a transmission line exhibits conspicuous "large displacement, small-strain" geometrically nonlinear characteristics. In particular, when induced by wind and rain or strong wind, transmission lines are more prone to relatively strong nonlinear vibration. In severe cases, such vibration can cause damage to transmission lines. Therefore, an in-depth investigation of the nonlinear vibration of HVOTLs is of great significance.

Research on cables has progressed from statics to dynamics and from the linear theory to the nonlinear theory [114]. In the static theory, cables are viewed as two-force bars with an aim to simplify calculation. As a result, the results of calculation based on the static theory differ relatively significantly from the actual situation $[1,2]$. In comparison, the natural frequencies of the out-of-plane and in-plane vibration of a suspended cable obtained based on the linear dynamic theory of cables are relatively accurate. Moreover, it can also be derived from the linear dynamic theory of cables that out-of-plane and in-plane modes of vibration are completely independent of one another [3]. However, cables are flexible 
structures; consequently, their motion exhibits significantly nonlinear motion characteristics and very complex forms that are a result of the combined action of external and internal resonance. In addition, the geometric and physical parameters of cables also have an impact on their dynamic response [4-14]. Evidently, even the linear dynamic theory cannot accurately describe the forms of motion of cables. It is irrefutable that the wind-induced vibration response of longspan, lightweight, and small-damping transmission lines has inherently nonlinear characteristics [15-24]. However, the current design specification still requires the use of the linear static theory of suspended cables to design transmission lines, which clearly cannot meet the engineering demand. The abovementioned linear design theory is easily mastered and applied in engineering, but its shortcomings are also obvious. For example, it assumes that the relationship between the static load and the response of the whole line is a linear function in calculations, which obviously does not conform to the nonlinear mechanical properties of a transmission line. Furthermore, there is a significant difference between the inplane and out-of-plane vibration of a transmission line, and the former has obvious nonlinearity stiffness that will induce additional tension of the transmission line. Therefore, it is not enough to calculate the response of a transmission line only by its first-order out-of-plane vibration. In fact, as a highly flexible line system, it is inevitable to consider the multiorder vibrations of a transmission line, and the dynamic tension generated by the vibration of the transmission line under the action of the pulsating wind is the main load that acts on the transmission tower.

Based on the aforementioned analysis, according to a generalized Hamilton's principle, three-dimensional (3D) nonlinear differential equations of motion for horizontally suspended transmission lines that consider the initial deflection are established in this work. Based on the characteristics of an actual transmission line, the 3D equations are simplified to two-dimensional (2D) equations. On this basis, the method of multiple scales is employed to theoretically study the nonlinear internal resonance behavior of transmission lines. Moreover, a higher-order Runge-Kutta method is also used to perform a numerical analysis of the nonlinear vibration characteristics of transmission lines to reveal the characteristics of the coupled action of the out-of-plane and in-plane vibration of transmission lines when in nonlinear vibration, with the aim of providing a basis for the reasonable design of HVOTLs.

\section{3D Nonlinear Equations of Motion for Elastic Transmission Lines}

2.1. Establishing $3 D$ Equations of Motion. Figure 1 shows an elastic transmission line with its two ends hinge-supported at the same height. The initial static equilibrium geometric configuration $S_{0}$ of the transmission line in the plane $O x y$ is used as the reference location and is represented by function $x(y) . d_{c}, L_{c}$, and $T_{c}(y, t)$ represent the sag and span of the dynamic tension in the transmission line, respectively. $S_{D}$ represents the $3 \mathrm{D}$ dynamic configuration of the transmission

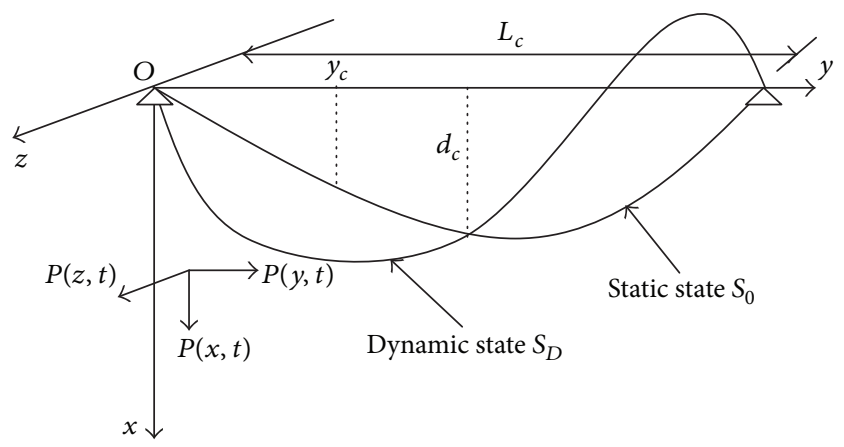

FIgURE 1: Spatial configuration of a transmission line.

line under an external excitation $P(t) . P(x, t), P(y, t)$, and $P(z, t)$ represent the components of the external excitation $P(t)$ in the $x$-, $y$-, and $z$-axes, respectively. $E_{c}$ and $A_{c}$ represent the elastic modulus and the cross-sectional area of the transmission line, respectively. $\gamma_{c}$ represents the dead weight of the transmission line per unit length $\left(\gamma_{c}=m_{c} g\right.$, where $g$ represents the acceleration of gravity). $u_{c}(y, t)$, $v_{c}(y, t)$, and $w_{c}(y, t)$ represent the displacements of the transmission line along the longitudinal in-plane vibration ( $y$-axis direction), the transverse in-plane vibration ( $x$-axis direction), and the transverse out-of-plane vibration ( $z$-axis direction), respectively.

To simplify analysis, the following assumptions [12, 25] are made: (a) the flexural, torsional, and shear stiffness of the transmission line is sufficiently low and thus negligible; (b) the transmission line only bears tension in the axial direction, and its axial strain, when vibrating, is sufficiently small and thus negligible; (c) only geometric nonlinearity is considered, whereas material nonlinearity is not considered.

When the tension in the transmission line is in the initial static equilibrium state, the length $d s_{0}$ of the differential element at any arbitrary location $y_{c}$ along the $y$-axis direction is

$$
d s_{0}=\sqrt{1+\left(\frac{\partial x}{\partial y}\right)^{2}} d y
$$

According to the definition of Lagrangian strain, initially, the length $d s$ of the differential element of the transmission line under the action of gravity is

$$
d s=\frac{\sqrt{1+(\partial x / \partial y)^{2}}}{1+\varepsilon_{c 0}} d y,
$$

where $\varepsilon_{c 0}$ represents the initial static strain of the transmission line, which is calculated using the following equation:

$$
\varepsilon_{c 0}=\frac{T_{c}(y, 0)}{E_{c} A_{c}}
$$

where $T_{c}(y, 0)$ represents the initial tension in the transmission line. 
The length of the differential element of the transmission line after it has undergone dynamic deformation under an external excitation $P(t)$ is

$$
d s_{D}=\sqrt{\left(1+\frac{\partial u_{c}}{\partial y}\right)^{2}+\left(\frac{\partial x}{\partial y}+\frac{\partial v_{c}}{\partial y}\right)^{2}+\left(\frac{\partial w_{c}}{\partial y}\right)^{2}} d y
$$

The total dynamic strain [12] of the transmission line in a motion state under the external excitation is

$$
\begin{aligned}
\varepsilon_{c} & =\frac{d s_{D}-d s}{d s}=\frac{1+\varepsilon_{c 0}}{\sqrt{1+(\partial x / \partial y)^{2}}} \\
& \cdot \sqrt{\left(1+\frac{\partial u_{c}}{\partial y}\right)^{2}+\left(\frac{\partial x}{\partial y}+\frac{\partial v_{c}}{\partial y}\right)^{2}+\left(\frac{\partial w_{c}}{\partial y}\right)^{2}}-1 .
\end{aligned}
$$

The transient total tension $T_{c}(y, t)$ in the differential element of the transmission line in a motion state is

$$
\begin{aligned}
& T_{c}(y, t)=T_{c}(y, 0)+E_{c} A_{c} \varepsilon_{c}=T_{c}(y, 0) \\
& +\left(\frac{1}{\sqrt{1+(\partial x / \partial y)^{2}}}\right. \\
& \left.+\sqrt{\left(1+\frac{\partial u_{c}}{\partial y}\right)^{2}+\left(\frac{\partial x}{\partial y}+\frac{\partial v_{c}}{\partial y}\right)^{2}+\left(\frac{\partial w_{c}}{\partial y}\right)^{2}}-1\right) \\
& \cdot E_{c} A_{c} .
\end{aligned}
$$

According to a generalized Hamilton's principle,

$$
\delta \int_{t_{1}}^{t_{2}}\left(K^{v}-U\right) d t+\int_{t_{1}}^{t_{2}} \delta W d t=0,
$$

where $K^{v}, U, \delta W$ represent the kinetic energy and the strain energy of the transmission line and the virtual work done by nonconservative forces (including the virtual work $\delta W_{c}$ done by the damping force and the virtual work $\delta W_{P}$ done by the external excitation), respectively.

The kinetic energy of the transmission line is

$$
K^{v}=\frac{1}{2} \int_{0}^{L_{c}} \frac{\gamma_{c} \sqrt{1+(\partial x / \partial y)^{2}}}{g\left(1+\varepsilon_{c 0}\right)}\left(\dot{u}_{c}^{2}+\dot{v}_{c}^{2}+\dot{w}_{c}^{2}\right) d y,
$$

where $\gamma_{c} \sqrt{1+(\partial x / \partial y)^{2}} / g\left(1+\varepsilon_{c 0}\right)$ represents the mass of the transmission line in the static equilibrium state per unit length and $\dot{u}_{c}, \dot{v}_{c}$, and $\dot{w}_{c}$ represent the vibration velocities of the transmission line in the $y^{-}, x-$, and $z$-axis directions, respectively.

According to the aforementioned assumptions, the strain energy of the transmission line can only be generated by an axial tension. According to the principle of virtual work, the strain energy is

$$
U=\int_{0}^{L c} T_{c}(y, 0) \varepsilon_{c} d s+\frac{1}{2} \int_{0}^{L c} E_{c} A_{c} \varepsilon_{c}^{2} d s .
$$

The virtual work done by the damping force is

$$
\delta W_{c}=-\int_{0}^{L_{c}} c_{c}\left(\dot{u}_{c} \delta u_{c}+\dot{v}_{c} \delta v_{c}+\dot{w}_{c} \delta w_{c}\right) d y
$$

where $c_{c}$ represents the structural damping of the transmission line.

The virtual work done by the external excitation $P(t)$ is

$$
\begin{aligned}
& \delta W_{P} \\
& \quad=\int_{0}^{L_{c}}\left(P(y, t) \delta u_{c}+P(x, t) \delta v_{c}+P(z, t) \delta w_{c}\right) d y .
\end{aligned}
$$

By substituting (8)-(11) into (7) and considering the boundary conditions, the following is satisfied at the locations $y_{c}=0$ and $y_{c}=L_{c}$ :

$$
\delta u_{c}=\delta v_{c}=\delta w_{c}=0
$$

Thus, the Euler equations for the transmission line along the virtual displacement $\delta u_{c}, \delta v_{c}$, and $\delta w_{c}$ directions are obtained:

$$
\begin{aligned}
& \frac{\partial}{\partial y}\left(\frac{E_{c} A_{c}\left(1+\partial u_{c} / \partial y\right)}{\sqrt{1+(\partial x / \partial y)^{2}}}\right. \\
& \left.+\frac{\left(T_{c}(y, 0)-E_{c} A_{c}\right)\left(1+\partial u_{c} / \partial y\right)}{\sqrt{\left(1+\partial u_{c} / \partial y\right)^{2}+\left(\partial x / \partial y+\partial v_{c} / \partial y\right)^{2}+\left(\partial w_{c} / \partial y\right)^{2}}}\right) \\
& =\frac{\gamma_{c} \sqrt{1+(\partial x / \partial y)^{2}}}{g\left(1+\varepsilon_{c 0}\right)} \ddot{u}_{c}+c_{c} \dot{u}_{c}-P(y, t), \\
& \frac{\partial}{\partial y}\left(\frac{E_{c} A_{c}\left(\partial x / \partial y+\partial v_{c} / \partial y\right)}{\sqrt{1+(\partial x / \partial y)^{2}}}\right. \\
& \left.+\frac{\left(T_{c}(y, 0)-E_{c} A_{c}\right)\left(\partial x / \partial y+\partial v_{c} / \partial y\right)}{\sqrt{\left(1+\partial u_{c} / \partial y\right)^{2}+\left(\partial x / \partial y+\partial v_{c} / \partial y\right)^{2}+\left(\partial w_{c} / \partial y\right)^{2}}}\right) \\
& =\frac{\gamma_{c} \sqrt{1+(\partial x / \partial y)^{2}}}{g\left(1+\varepsilon_{c 0}\right)} \ddot{v}_{c}+c_{c} \dot{v}_{c}-P(x, t), \\
& \frac{\partial}{\partial y}\left(\frac{E_{c} A_{c}\left(\partial w_{c} / \partial y\right)}{\sqrt{1+(\partial x / \partial y)^{2}}}\right. \\
& \left.+\frac{\left(T_{c}(y, 0)-E_{c} A_{c}\right)\left(\partial w_{c} / \partial y\right)}{\sqrt{\left(1+\partial u_{c} / \partial y\right)^{2}+\left(\partial x / \partial y+\partial v_{c} / \partial y\right)^{2}+\left(\partial w_{c} / \partial y\right)^{2}}}\right) \\
& =\frac{\gamma_{c} \sqrt{1+(\partial x / \partial y)^{2}}}{g\left(1+\varepsilon_{c 0}\right)} \ddot{w}_{c}+c_{c} \dot{w}_{c}-P(z, t) \text {. }
\end{aligned}
$$

Because the sag-to-span ratio $d_{c} / L_{c}$ of a transmission line is generally very small, $\partial u_{c} / \partial y \ll 1, \partial v_{c} / \partial y \ll 1, \partial w_{c} / \partial y \ll 1$, 
and $\partial x / \partial y \ll 1$ under small-strain conditions. Thus, these small quantities can each be expanded in a Taylor series, and their higher-order terms are negligible. In other words, in the abovementioned equations,

$$
\begin{gathered}
\frac{1}{\sqrt{\left(1+\partial u_{c} / \partial y\right)^{2}+\left(\partial x / \partial y+\partial v_{c} / \partial y\right)^{2}+\left(\partial w_{c} / \partial y\right)^{2}}} \\
\approx 1-\left\{\frac{\partial u_{c}}{\partial y}-\left(\frac{\partial u_{c}}{\partial y}\right)^{2}+\frac{\partial x}{\partial y} \cdot \frac{\partial v_{c}}{\partial y}+\frac{1}{2}\left(\frac{\partial x}{\partial y}\right)^{2}\right. \\
\left.+\frac{1}{2}\left(\frac{\partial v_{c}}{\partial y}\right)^{2}+\frac{1}{2}\left(\frac{\partial w_{c}}{\partial y}\right)^{2}\right\} .
\end{gathered}
$$

Let

$$
\begin{aligned}
\eta= & \frac{\partial u_{c}}{\partial y}-\left(\frac{\partial u_{c}}{\partial y}\right)^{2}+\frac{\partial x}{\partial y} \cdot \frac{\partial v_{c}}{\partial y}+\frac{1}{2}\left(\frac{\partial x}{\partial y}\right)^{2} \\
& +\frac{1}{2}\left(\frac{\partial v_{c}}{\partial y}\right)^{2}+\frac{1}{2}\left(\frac{\partial w_{c}}{\partial y}\right)^{2}
\end{aligned}
$$

By substituting (15) into (13), nonlinear equations of motion for the elastic transmission line that is horizontally hingesupported at the two ends are obtained:

$$
\begin{aligned}
& \frac{\partial}{\partial y}\left\{\left[\frac{E_{c} A_{c}}{\sqrt{1+(\partial x / \partial y)^{2}}}+\left(T_{c}(y, 0)-E_{c} A_{c}\right)(1-\eta)\right]\right. \\
& \left.\cdot\left(1+\frac{\partial u_{c}}{\partial y}\right)\right\}=\frac{\gamma_{c} \sqrt{1+(\partial x / \partial y)^{2}}}{g\left(1+\varepsilon_{c 0}\right)} \ddot{u}_{c}+c_{c} \dot{u}_{c} \\
& -P(y, t) \text {, } \\
& \frac{\partial}{\partial y}\left\{\left[\frac{E_{c} A_{c}}{\sqrt{1+(\partial x / \partial y)^{2}}}+\left(T_{c}(y, 0)-E_{c} A_{c}\right)(1-\eta)\right]\right. \\
& \left.\cdot\left(\frac{\partial x}{\partial y}+\frac{\partial v_{c}}{\partial y}\right)\right\}=\frac{\gamma_{c} \sqrt{1+(\partial x / \partial y)^{2}}}{g\left(1+\varepsilon_{c 0}\right)} \ddot{v}_{c}+c_{c} \dot{v}_{c} \\
& -P(x, t) \text {, } \\
& \frac{\partial}{\partial y}\left\{\left[\frac{E_{c} A_{c}}{\sqrt{1+(\partial x / \partial y)^{2}}}\right.\right. \\
& \left.\left.+\left(T_{c}(y, 0)-E_{c} A_{c}\right)(1-\eta)\right] \frac{\partial w_{c}}{\partial y}\right\} \\
& =\frac{\gamma_{c} \sqrt{1+(\partial x / \partial y)^{2}}}{g\left(1+\varepsilon_{c 0}\right)} \ddot{w}_{c}+c_{c} \dot{w}_{c}-P(z, t) .
\end{aligned}
$$

In (16)-(18), $\ddot{u}_{c}, \ddot{v}_{c}, \ddot{w}_{c}$ represent the accelerations of vibration of the transmission line along the $y_{-}, x$-, and $z$-axes, respectively.

The above set of partial differential equations contains all the main vibration characteristics of the initial elastic transmission line continuum system, that is, the quadratic and cubic coupling terms associated with the initial deflection, the axial tension, and the spatial motion, which can be used to analyze the nonlinear dynamic behavior of the transmission line.

2.2. Equations of Coupled In-Plane and Out-of-Plane Motion for a Hinge-Supported Transmission Line. Considering that the axial stiffness $E_{c} A_{c}$ of a transmission line is generally relatively high and its axial elongation due to vibration is far smaller than its transverse vibration amplitude, the axial inertia force of a transmission line when in vibration is negligible. Under normal circumstances, the axial damping of a transmission line is relatively small; thus, the axial damping force of a transmission line when in motion is negligible. In addition, in Figure 1, since there is no external excitation along the axial direction of the transmission line $(y$-axis direction), $P(y, t)=0$. Thus, (16) can be rewritten as

$$
\begin{aligned}
& \frac{\partial}{\partial y}\left\{\left[\frac{E_{c} A_{c}}{\sqrt{1+(\partial x / \partial y)^{2}}}+\left(T_{c}(y, 0)-E_{c} A_{c}\right)(1-\eta)\right]\right. \\
& \left.\cdot\left(1+\frac{\partial u_{c}}{\partial y}\right)\right\}=0 .
\end{aligned}
$$

The following approximation relation is then used:

$$
\frac{1}{\sqrt{1+(\partial x / \partial y)^{2}}} \approx 1-\frac{1}{2}\left(\frac{\partial x}{\partial y}\right)^{2}
$$

By substituting (20) into (19), integrating the two sides of (19) over the span $\left[0, L_{c}\right]$ of the transmission line once, and ignoring the terms unrelated to time $t$, we have

$$
\begin{gathered}
T_{c}(y, 0)\left[1-\frac{1}{2}\left(\frac{\partial x}{\partial y}\right)^{2}\right] \cdot \frac{\partial u_{c}}{\partial y}-\left(T_{c}(y, 0)-E_{c} A_{c}\right) \eta \\
-\left(T_{c}(y, 0)-E_{c} A_{c}\right) \eta \cdot \frac{\partial u_{c}}{\partial y}=\hbar(t),
\end{gathered}
$$

where $\hbar(t)$ is an arbitrary function. By dividing the two sides of (21) by $E_{c} A_{c}$ and considering that the initial tension $T_{c}(y, 0)$ in a transmission line is generally far smaller than its axial stiffness $E_{c} A_{c}$ and that the axial deformation of a transmission line is a higher-order small quantity compared to its vertical in-plane deformation and out-of-plane deformation, we have

$$
\frac{\partial u_{c}}{\partial y}+\frac{\partial x}{\partial y} \cdot \frac{\partial v_{c}}{\partial y}+\frac{1}{2}\left(\frac{\partial w_{c}}{\partial y}\right)^{2}+\frac{1}{2}\left(\frac{\partial v_{c}}{\partial y}\right)^{2} \approx \frac{\hbar(t)}{E_{c} A_{c}}
$$


To determine $\hbar(t)$, the two sides of (22) are integrated over the interval $\left[0, L_{c}\right]$ and the transmission line boundary conditions represented by (23) are used. After simplification, we have (24).

$$
\begin{aligned}
& u_{c}(0, t)=0, \\
& u_{c}\left(L_{c}, t\right)=0, \\
& \frac{\hbar(t)}{E_{c} A_{c}} \cdot L_{c}=\int_{0}^{L_{c}}\left[\frac{\partial u_{c}}{\partial y}+\frac{\partial x}{\partial y} \cdot \frac{\partial v_{c}}{\partial y}+\frac{1}{2}\left(\frac{\partial w_{c}}{\partial y}\right)^{2}\right. \\
& \left.\quad+\frac{1}{2}\left(\frac{\partial v_{c}}{\partial y}\right)^{2}\right] d y .
\end{aligned}
$$

Let us set

$$
\begin{gathered}
\lambda(t)=\frac{\hbar(t)}{E_{c} A_{c}}=\frac{1}{L_{c}} \int_{0}^{L_{c}}\left[\frac{\partial u_{c}}{\partial y}+\frac{\partial x}{\partial y} \cdot \frac{\partial v_{c}}{\partial y}\right. \\
\left.+\frac{1}{2}\left(\frac{\partial w_{c}}{\partial y}\right)^{2}+\frac{1}{2}\left(\frac{\partial v_{c}}{\partial y}\right)^{2}\right] d y .
\end{gathered}
$$

By substituting (22) and (25) into (17) and (18), respectively, we have

$$
\begin{gathered}
\frac{\partial}{\partial y}\left(T_{c}(y, 0) \cdot\left[1-\frac{1}{2}\left(\frac{\partial x}{\partial y}\right)^{2}\right] \cdot \frac{\partial v_{c}}{\partial y}\right. \\
\left.-\left(T_{c}(y, 0)-E_{c} A_{c}\right) \cdot \lambda(t) \cdot \frac{\partial x}{\partial y}\right) \\
-\frac{\partial}{\partial y}\left(\left(T_{c}(y, 0)-E_{c} A_{c}\right) \cdot \lambda(t) \cdot \frac{\partial v_{c}}{\partial y}\right) \\
\quad=\frac{\gamma_{c} \sqrt{1+(\partial x / \partial y)^{2}}}{g\left(1+\varepsilon_{c 0}\right)} \ddot{v}_{c}+c_{c} \dot{v}_{c}-P(x, t), \\
\frac{\partial}{\partial y}\left(T_{c}(y, 0) \cdot\left[1-\frac{1}{2}\left(\frac{\partial x}{\partial y}\right)^{2}\right] \cdot \frac{\partial w_{c}}{\partial y}\right. \\
\left.-\left(T_{c}(y, 0)-E_{c} A_{c}\right) \cdot \lambda(t) \cdot \frac{\partial w_{c}}{\partial y}\right) \\
\quad=\frac{\gamma_{c} \sqrt{1+(\partial x / \partial y)^{2}}}{g\left(1+\varepsilon_{c 0}\right)} \ddot{w}_{c}+c_{c} \dot{w}_{c}-P(z, t) .
\end{gathered}
$$

For ease of analysis, by use of Galerkin's modal truncation method, the in-plane and out-of-plane response of the transmission line is represented by

$$
\begin{aligned}
& v_{c}(y, t)=\sum_{i=1}^{N} \varphi_{i}(y) \cdot q_{i}(t), \\
& w_{c}(y, t)=\sum_{i=1}^{N} \varphi_{i}(y) \cdot q_{i}(t) .
\end{aligned}
$$

In (28), $\varphi_{i}(y)=\sin \left(i \pi y / L_{c}\right)$ is the $i$ th-order vibration mode function (the two ends of the conductor line are considered to be hinge-connected), $N$ represents the retained mode order, and $q_{i}(t)$ represents the generalized coordinates. By substituting it into (26) and (27), (28) can be transformed to ordinary differential equations of motion, which are subsequently solved. Equation (28) is subjected to first-order model truncation using Galerkin's modal truncation method; that is, set

$$
\begin{aligned}
& v_{c}(y, t)=\varphi_{v}(y) \cdot q_{v}(t)=\sin \left(\frac{\pi y}{L_{c}}\right) \cdot q_{v}(t), \\
& w_{c}(y, t)=\varphi_{w}(y) \cdot q_{w}(t)=\sin \left(\frac{\pi y}{L_{c}}\right) \cdot q_{w}(t) .
\end{aligned}
$$

By substituting (29) into (26) and (27), subsequently multiplying the two sides of $(26)$ by $\varphi_{v}(y)$, integrating the resulting equation over the interval $\left[0, L_{c}\right]$, multiplying the two sides of (27) by $\varphi_{w}(y)$, and integrating the resultant equation over the interval $\left[0, L_{c}\right]$, discrete equations of coupled inplane and out-of-plane motion for the transmission line are obtained:

$$
\begin{aligned}
& a_{1} \ddot{q}_{v}(t)+a_{2} q_{v}(t)+a_{3} q_{v}^{2}(t)+a_{4} q_{w}^{2}(t)+a_{5} q_{v}^{3}(t) \\
& \quad+a_{6} q_{w}^{2}(t) q_{v}(t)+a_{7} \dot{q}_{v}(t)=P_{v}, \\
& b_{1} \ddot{q}_{w}(t)+b_{2} q_{w}(t)+b_{3} q_{v}(t) q_{w}(t)+b_{4} q_{v}^{2}(t) q_{w}(t) \\
& \quad+b_{5} q_{w}^{3}(t)+b_{6} \dot{q}_{w}(t)=P_{w} .
\end{aligned}
$$

The factors in (30) are calculated as follows:

$$
\begin{aligned}
a_{1} & =\int_{0}^{L_{c}} \frac{\gamma_{c} \sqrt{1+(\partial x / \partial y)^{2}}}{g\left(1+\varepsilon_{c 0}\right)} \varphi_{v}^{2}(y) d y, \\
a_{2} & =-\int_{0}^{L_{c}} \frac{\partial}{\partial y}\left[T_{c}(y, 0) \cdot\left(1-\frac{1}{2}\left(\frac{\partial x}{\partial y}\right)^{2}\right) \cdot \varphi_{v}^{\prime}(y)\right. \\
& \left.-\left(\frac{T_{c}(y, 0)-E_{c} A_{c}}{L_{c}}\right) \cdot \frac{\partial x}{\partial y} \cdot \int_{0}^{L_{c}} \frac{\partial x}{\partial y} \cdot \varphi_{v}^{\prime}(y) d y\right] \\
& \cdot \varphi_{v}(y) d y, \\
a_{3} & =\int_{0}^{L_{c}} \frac{\partial}{\partial y}\left[\left(\frac{T_{c}(y, 0)-E_{c} A_{c}}{2 L_{c}}\right) \cdot \frac{\partial x}{\partial y}\right. \\
& \cdot \int_{0}^{L_{c}} \varphi_{v}^{\prime 2}(y) d y+\left(\frac{T_{c}(y, 0)-E_{c} A_{c}}{L_{c}}\right) \\
& \left.\cdot \varphi_{v}^{\prime}(y) \int_{0}^{L_{c}} \frac{\partial x}{\partial y} \cdot \varphi_{v}^{\prime}(y) d y\right] \varphi_{v}(y) d y, \\
a_{4} & =\int_{0}^{L_{c}} \frac{\partial}{\partial y}\left[\left(\frac{T_{c}(y, 0)-E_{c} A_{c}}{2 L_{c}}\right) \cdot \frac{\partial x}{\partial y}\right. \\
& \left.\cdot \int_{0}^{L_{c}} \varphi_{w}^{\prime 2}(y) d y\right] \varphi_{v}(y) d y,
\end{aligned}
$$




$$
\begin{array}{cl}
a_{5}=\int_{0}^{L_{c}} \frac{\partial}{\partial y}\left[\left(\frac{T_{c}(y, 0)-E_{c} A_{c}}{2 L_{c}}\right) \cdot \varphi_{v}^{\prime}(y)\right. & \begin{array}{l}
\text { 3. Theoretical Analysis of the } \\
\text { Characteristics of the Nonlinear Coupled } \\
\text { In-Plane and Out-of-Plane Internal }
\end{array} \\
\left.\cdot \int^{L_{c}} \varphi_{v}^{\prime 2}(y) d y\right] \varphi_{v}(y) d y, & \text { Resonance of Transmission Lines }
\end{array}
$$

\section{Theoretical Analysis of the Characteristics of the Nonlinear Coupled In-Plane and Out-of-Plane Internal Resonance of Transmission Lines}$$
\left.\cdot \int_{0}^{L_{c}} \varphi_{v}^{\prime 2}(y) d y\right] \varphi_{v}(y) d y
$$$$
a_{6}=\int_{0}^{L_{c}} \frac{\partial}{\partial y}\left[\left(\frac{T_{c}(y, 0)-E_{c} A_{c}}{2 L_{c}}\right) \cdot \varphi_{v}^{\prime}(y)\right.
$$$$
\left.\cdot \int_{0}^{L_{c}} \varphi_{w}^{\prime 2}(y) d y\right] \varphi_{v}(y) d y
$$$$
a_{7}=\int_{0}^{L_{c}} c_{c} \varphi_{v}^{2}(y) d y,
$$$$
P_{v}=\int_{0}^{L_{c}} P(x, t) \varphi_{v}(y) d y,
$$$$
b_{1}=\int_{0}^{L_{c}} \frac{\gamma_{c} \sqrt{1+(\partial x / \partial y)^{2}}}{g\left(1+\varepsilon_{c 0}\right)} \varphi_{w}^{2}(y) d y \text {, }
$$$$
b_{2}=-\int_{0}^{L_{c}} \frac{\partial}{\partial y}\left[T_{c}(y, 0) \cdot\left(1-\frac{1}{2}\left(\frac{\partial x}{\partial y}\right)^{2}\right) \cdot \varphi_{w}^{\prime}(y)\right]
$$$$
\cdot \varphi_{w}(y) d y \text {, }
$$$$
b_{3}=\int_{0}^{L_{c}} \frac{\partial}{\partial y}\left[\left(\frac{T_{c}(y, 0)-E_{c} A_{c}}{L_{c}}\right) \cdot \varphi_{w}^{\prime}(y)\right.
$$$$
\left.\cdot \int_{0}^{L_{c}} \frac{\partial x}{\partial y} \cdot \varphi_{v}^{\prime}(y) d y\right] \varphi_{w}(y) d y,
$$$$
b_{4}=\int_{0}^{L_{c}} \frac{\partial}{\partial y}\left[\left(\frac{T_{c}(y, 0)-E_{c} A_{c}}{2 L_{c}}\right) \cdot \varphi_{w}^{\prime}(y)\right.
$$$$
\left.\cdot \int_{0}^{L_{c}} \varphi_{v}^{\prime 2}(y) d y\right] \varphi_{w}(y) d y,
$$$$
b_{5}=\int_{0}^{L_{c}} \frac{\partial}{\partial y}\left[\left(\frac{T_{c}(y, 0)-E_{c} A_{c}}{2 L_{c}}\right) \cdot \varphi_{w}^{\prime}(y)\right.
$$$$
\left.\cdot \int_{0}^{L_{c}} \varphi_{w}^{\prime 2}(y) d y\right] \varphi_{w}(y) d y,
$$$$
b_{6}=\int_{0}^{L_{c}} c_{c} \varphi_{w}^{2}(y) d y,
$$$$
P_{w}=\int_{0}^{L_{c}} P(z, t) \varphi_{w}(y) d y .
$$

As demonstrated in (30), the in-plane and out-of-plane motion of the transmission line exhibits cubic nonlinearity, whereas the coupled in-plane and out-of-plane motion exhibits linearity, quadratic nonlinearity, and cubic nonlinearity; that is, the nonlinear coupling is very strong.
To further study the nonlinear coupled in-plane and out-ofplane dynamic characteristics of an overhead transmission line, we conducted the following analysis with the help of the multiple-scale (MS) method [26-29], which is an effective method for the analysis of nonlinear dynamics.

Assume that the external excitation is a harmonic excitation: that is,

$$
\begin{aligned}
& P(x, t)=A_{v} \cos \left(\Omega_{x} t\right), \\
& P(z, t)=A_{w} \cos \left(\Omega_{z} t\right),
\end{aligned}
$$

where $A_{v}$ and $A_{w}$ represent the amplitudes of the in-plane excitation and the out-of-plane excitation, respectively, on the transmission line, and $\Omega_{x}$ and $\Omega_{z}$ represent the circular frequencies of the in-plane excitation and the out-of-plane excitation, respectively.

Here, the in-plane excitation on the transmission line is not considered, that is, the generalized in-plane excitation load $P_{v}$ is assumed to be zero. Now, the generalized out-ofplane excitation load on the transmission line is set to $P_{w}=$ $P_{0} \cos \Omega_{z} t$. In addition, the parameters $\varepsilon, \alpha_{1}, \alpha_{2}, \mu_{1}, \mu_{2}, \kappa_{1}$, $\kappa_{2}, \beta_{1}, \beta_{2}, \gamma_{1}$, and $f_{2}$ are introduced to transform (30) to dimensionless equations:

$$
\begin{aligned}
& \ddot{q}_{v}(t)+\omega_{1}^{2} q_{v}(t)+\varepsilon \alpha_{1} q_{v}^{2}(t)+\varepsilon \kappa_{1} q_{w}^{2}(t)+\varepsilon \beta_{1} q_{v}^{3}(t) \\
& \quad+\varepsilon \gamma_{1} q_{w}^{2}(t) q_{v}(t)+2 \varepsilon \mu_{1} \dot{q}_{v}(t)=0, \\
& \ddot{q}_{w}(t)+\omega_{2}^{2} q_{w}(t)+\varepsilon \alpha_{2} q_{v}(t) q_{w}(t)+\varepsilon \kappa_{2} q_{v}^{2}(t) q_{w}(t) \\
& \quad+\varepsilon \beta_{2} q_{w}^{3}(t)+2 \varepsilon \mu_{2} \dot{q}_{w}(t)=2 \varepsilon f_{2} \cos \left(\Omega_{z} t\right),
\end{aligned}
$$

where $\omega_{1}$ and $\omega_{2}$ represent the circular frequencies of the in-plane and out-of-plane vibrations of the transmission line, respectively. The abovementioned parameters have the following conversion relationships:

$$
\begin{aligned}
\omega_{1}^{2} & =\frac{a_{2}}{a_{1}}, \\
2 \varepsilon \mu_{1} & =\frac{a_{7}}{a_{1}}, \\
\varepsilon \alpha_{1} & =\frac{a_{3}}{a_{1}}, \\
\varepsilon \kappa_{1} & =\frac{a_{4}}{a_{1}}, \\
\varepsilon \beta_{1} & =\frac{a_{5}}{a_{1}}, \\
\varepsilon \gamma_{1} & =\frac{a_{6}}{a_{1}}, \\
\omega_{2}^{2} & =\frac{b_{2}}{b_{1}},
\end{aligned}
$$




$$
\begin{aligned}
2 \varepsilon \mu_{2} & =\frac{b_{6}}{b_{1}}, \\
\varepsilon \alpha_{2} & =\frac{b_{3}}{b_{1}}, \\
\varepsilon \kappa_{2} & =\frac{b_{4}}{b_{1}}, \\
\varepsilon \beta_{2} & =\frac{b_{5}}{b_{1}}, \\
2 \varepsilon f_{2} & =\frac{P_{0}}{b_{1}} .
\end{aligned}
$$

The abovementioned set of nonlinear equations can be solved using the MS method, the basic idea of which is to regard the expansion of the response as a function with multiple independent variables (or multiple scales) [26].

The following independent variables are introduced:

$$
T_{n}=\varepsilon^{n} t \quad(n=0,1, \ldots),
$$

that is,

$$
\begin{aligned}
& T_{0}=t, \\
& T_{1}=\varepsilon t, \ldots
\end{aligned}
$$

Thus, the derivative with respect to $t$ is transformed to the expansion of a partial derivative with respect to $T_{n}$, that is,

$$
\begin{aligned}
\frac{d}{d t} & =\frac{d T_{0}}{d t} \frac{\partial}{\partial T_{0}}+\frac{d T_{1}}{d t} \frac{\partial}{\partial T_{1}}+\cdots \\
& =D_{0}+\varepsilon D_{1}+\varepsilon^{2} D_{2}+\cdots \\
\frac{d^{2}}{d t^{2}} & =\frac{d T_{0}}{d t} \frac{\partial^{2}}{\partial T_{0}^{2}}+2 \varepsilon \frac{\partial^{2}}{\partial T_{0} \partial T_{1}}+\cdots \\
& =D_{0}^{2}+2 \varepsilon D_{0} D_{1}+\varepsilon^{2}\left(D^{2}{ }_{1}+2 D_{1} D_{2}\right)+\cdots
\end{aligned}
$$

Hence, the solutions to (33) can be expressed as follows:

$$
\begin{aligned}
q_{v}(t, \varepsilon)= & q_{v 0}\left(T_{0}, T_{1}, T_{2}, \ldots\right)+\varepsilon q_{v 1}\left(T_{0}, T_{1}, T_{2}, \ldots\right) \\
& +\varepsilon^{2} q_{v 2}\left(T_{0}, T_{1}, T_{2}, \ldots\right)+\cdots \\
q_{w}(t, \varepsilon)= & q_{w 0}\left(T_{0}, T_{1}, T_{2}, \ldots\right)+\varepsilon q_{w 1}\left(T_{0}, T_{1}, T_{2}, \ldots\right) \\
& +\varepsilon^{2} q_{w 2}\left(T_{0}, T_{1}, T_{2}, \ldots\right)+\cdots,
\end{aligned}
$$

By substituting (37) and (38) into (33), we have

$$
\begin{aligned}
& \left(D_{1}^{2} q_{v 2}+2 \mu_{1} D_{1} q_{v 2}+2 \mu_{1} D_{2} q_{v 1}+2 D_{0} D_{1} q_{v 3}\right. \\
& \quad+2 \mu_{1} D_{0} q_{v 3}+\beta_{1} q_{v 1}^{3}+2 \alpha_{1} q_{v 1} q_{v 2}+6 \beta_{1} q_{v 0} q_{v 1} q_{v 2}
\end{aligned}
$$

$$
\begin{aligned}
& +2 \alpha_{1} q_{v 0} q_{v 3}+3 \beta_{1} q_{v 0}^{2} q_{v 3}+\gamma_{1} q_{w 0}^{2} q_{v 3} \\
& +2 \gamma_{1} q_{v 2} q_{w 0} q_{w 1}+\gamma_{1} q_{w 1}^{2} q_{v 1}+2 \gamma_{1} q_{v 1} q_{w 0} q_{w 2} \\
& +2 \kappa_{1} q_{w 1} q_{w 2}+2 \gamma_{1} q_{v 0} q_{w 1} q_{w 2}+2 \kappa_{1} q_{w 0} q_{w 3} \\
& \left.+2 \gamma_{1} q_{v 0} q_{w 0} q_{w 3}\right) \varepsilon^{4}+\left(D_{0}^{2} q_{v 3}+\omega_{1}^{2} q_{v 3}+2 \mu_{1} D_{2} q_{v 0}\right. \\
& +D_{1}^{2} q_{v 1}+2 \mu_{1} D_{1} q_{v 1}+2 D_{0} D_{1} q_{v 2}+2 \mu_{1} D_{0} q_{v 2} \\
& +\alpha_{1} q_{v 1}^{2}+3 \beta_{1} q_{v 0} q_{v 1}^{2}+2 \alpha_{1} q_{v 0} q_{v 2}+3 \beta_{1} q_{v 0}^{2} q_{v 2} \\
& +\gamma_{1} q_{v 2} q_{w 0}^{2}+2 \gamma_{1} q_{v 1} q_{w 0} q_{w 1}+\kappa_{1} q_{w 1}^{2}+\gamma_{1} q_{v 0} q_{w 1}^{2} \\
& \left.+2 \kappa_{1} q_{w 0} q_{w 2}+2 \gamma_{1} q_{v 0} q_{w 0} q_{w 2}\right) \varepsilon^{3}+\left(D_{0}^{2} q_{v 2}+\omega_{1}^{2} q_{v 2}\right. \\
& +D_{1}^{2} q_{v 0}+2 \mu_{1} D_{0} q_{v 0}+2 D_{0} D_{1} q_{v 1}+2 \mu_{1} D_{0} q_{v 1} \\
& +3 \beta_{1} q_{v 0}^{2} q_{v 1}+2 \kappa_{1} q_{w 0} q_{w 1}+\gamma_{1} q_{w 0}^{2} q_{v 1} \\
& \left.+2 \gamma_{1} q_{w 0} q_{w 1} q_{v 0}+2 \alpha_{1} q_{v 0} q_{v 1}\right) \varepsilon^{2}+\left(D_{0}^{2} q_{v 1}+\omega_{1}^{2} q_{v 1}\right. \\
& +2 D_{0} D_{1} q_{v 0}+2 \mu_{1} D_{0} q_{v 0}+\alpha_{1} q_{v 0}^{2}+\kappa_{1} q_{w 0}^{2}+\beta_{1} q_{v 0}^{3} \\
& \left.+\gamma_{1} q_{w 0}^{2} q_{v 0}\right) \varepsilon+D_{0}^{2} q_{v 0}+\omega_{1}^{2} q_{v 0}=0
\end{aligned}
$$$$
\left(D_{1}^{2} q_{w 2}+2 \mu_{2} D_{1} q_{w 2}+2 \mu_{2} D_{0} q_{w 3}+2 D_{0} D_{1} q_{w 3}\right.
$$$$
+2 \mu_{2} D_{2} q_{w 1}+\beta_{2} q_{w 1}^{3}+\alpha_{2} q_{v 3} q_{w 0}+2 \kappa_{2} q_{v 0} q_{v 3} q_{w 0}
$$$$
+2 \kappa_{2} q_{v 1} q_{v 2} q_{w 0}+\kappa_{2} q_{v 1}^{2} q_{w 1}+\alpha_{2} q_{v 2} q_{w 1}
$$$$
+2 \kappa_{2} q_{v 0} q_{v 2} q_{w 1}+\alpha_{2} q_{v 1} q_{w 2}+2 \kappa_{2} q_{v 0} q_{v 1} q_{w 2}
$$$$
+6 \beta_{2} q_{w 0} q_{w 1} q_{w 2}+\alpha_{2} q_{v 0} q_{w 3}+\kappa_{2} q_{v 0}^{2} q_{w 3}
$$$$
\left.+3 \beta_{2} q_{w 0}^{2} q_{w 3}\right) \varepsilon^{4}+\left(D_{0}^{2} q_{w 3}+\omega_{2}^{2} q_{w 3}+2 \mu_{2} D_{2} q_{w 0}\right.
$$$$
+D_{1}^{2} q_{w 1}+2 \mu_{2} D_{1} q_{w 1}+2 D_{0} D_{1} q_{w 2}+2 \mu_{2} D_{0} q_{w 2}
$$$$
+\alpha_{2} q_{v 2} q_{w 0}+\kappa_{2} q_{v 1}^{2} q_{w 0}+3 \beta_{2} q_{w 0} q_{w 1}^{2}+\alpha_{2} q_{v 1} q_{w 1}
$$$$
+3 \beta_{2} q_{w 0}^{2} q_{w 2}+2 \kappa_{2} q_{v 0} q_{v 2} q_{w 0}+2 \kappa_{2} q_{v 0} q_{v 1} q_{w 1}
$$$$
\left.+\kappa_{2} q_{v 0}^{2} q_{w 0}+\alpha_{2} q_{v 0} q_{w 2}\right) \varepsilon^{3}+\left(D_{0}^{2} q_{w 2}+\omega_{2}^{2} q_{w 2}\right.
$$$$
+D_{1}^{2} q_{w 0}+2 \mu_{2} D_{1} q_{w 0}+2 D_{0} D_{1} q_{w 2}+2 \mu_{2} D_{0} q_{w 2}
$$$$
+D_{1}^{2} q_{w 1}+2 \mu_{2} D_{1} q_{w 1}+3 \beta_{2} q_{w 0}^{2} q_{w 2}+3 \beta_{2} q_{w 0} q_{w 1}^{2}
$$$$
+2 \kappa_{2} q_{v 0} q_{v 1} q_{w 1}+2 \kappa_{2} q_{v 0} q_{v 2} q_{w 0}+\alpha_{2} q_{v 2} q_{w 0}
$$$$
\left.+\alpha_{2} q_{v 1} q_{w 1}+\alpha_{2} q_{v 0} q_{w 2}+\kappa_{2} q_{v 0}^{2} q_{w 2}\right) \varepsilon^{2}+\left(D_{0}^{2} q_{w 1}\right.
$$$$
+\omega_{2}^{2} q_{w 1}+2 D_{0} D_{1} q_{w 0}+2 \mu_{2} D_{0} q_{w 0}+\alpha_{2} q_{v 0} q_{w 0}
$$$$
\left.+\kappa_{2} q_{v 0}^{2} q_{w 0}+\beta_{2} q_{w 0}^{3}\right) \varepsilon+D_{0}^{2} q_{w 0}+\omega_{2}^{2} q_{w 0}=2 \varepsilon f_{2}
$$$$
\cdot \cos \left(\Omega_{z} t\right) \text {. }
$$ 
By setting the sum of the coefficients of the same power of $\varepsilon$ to zero, we have

$\varepsilon^{0}$ :

$$
\begin{aligned}
D_{0}{ }^{2} q_{v 0}+\omega_{1}^{2} q_{v 0} & =0, \\
D_{0}^{2} q_{w 0}+\omega_{2}^{2} q_{w 0} & =0,
\end{aligned}
$$

$\varepsilon^{1}:$

$$
\begin{aligned}
& D_{0}^{2} q_{v 1}+2 D_{0} D_{1} q_{v 0}+2 \mu_{1} D_{0} q_{v 0}+\omega_{1}^{2} q_{v 1}+\beta_{1} q_{v 0}^{3} \\
& \quad+a_{1} q_{v 0}^{2}+\gamma_{1} q_{w 0}^{2} q_{v 0}+\kappa_{1} q_{w 0}^{2}=0 \\
& D_{0}^{2} q_{w 1}+2 D_{0} D_{1} q_{w 0}+\beta_{2} q_{w 0}^{3}+\omega_{2}^{2} q_{w 1}+a_{2} q_{v 0} q_{w 0} \\
& \quad+2 \mu_{2} D_{0} q_{w 0}+\kappa_{2} q_{v 0}^{2} q_{w 0}=2 f_{2} \cos \left(\Omega_{z} t\right)
\end{aligned}
$$

$\varepsilon^{2}:$

$$
\begin{aligned}
& D_{0}^{2} q_{v 2}+\omega_{1}^{2} q_{v 2}+D_{1}^{2} q_{v 0}+2 \mu_{1} D_{0} q_{v 0}+2 D_{0} D_{1} q_{v 1} \\
& \quad+2 \mu_{1} D_{0} q_{v 1}+3 \beta_{1} q_{v 0}^{2} q_{v 1}+2 \kappa_{1} q_{w 0} q_{w 1}+\gamma_{1} q_{w 0}^{2} q_{v 1} \\
& \quad+2 \gamma_{1} q_{w 0} q_{w 1} q_{v 0}+2 \alpha_{1} q_{v 0} q_{v 1}=0 \\
& D_{0}^{2} q_{w 2}+\omega_{2}^{2} q_{w 2}+D_{1}^{2} q_{w 0}+2 \mu_{2} D_{1} q_{w 0}+2 D_{0} D_{1} q_{w 2} \\
& \quad+2 \mu_{2} D_{0} q_{w 2}+D_{1}^{2} q_{w 1}+2 \mu_{2} D_{1} q_{w 1}+3 \beta_{2} q_{w 0}^{2} q_{w 2} \\
& \quad+3 \beta_{2} q_{w 0} q_{w 1}^{2}+2 \kappa_{2} q_{v 0} q_{v 1} q_{w 1}+2 \kappa_{2} q_{v 0} q_{v 2} q_{w 0} \\
& \quad+\alpha_{2} q_{v 2} q_{w 0}+\alpha_{2} q_{v 1} q_{w 1}+\alpha_{2} q_{v 0} q_{w 2} \\
& \quad+\kappa_{2} q_{v 0}^{2} q_{w 2}=0,
\end{aligned}
$$

$\varepsilon^{3}:$

$$
\begin{aligned}
& D_{0}^{2} q_{v 3}+\omega_{1}^{2} q_{v 3}+2 \mu_{1} D_{2} q_{v 0}+D_{1}^{2} q_{v 1}+2 \mu_{1} D_{1} q_{v 1} \\
& \quad+2 D_{0} D_{1} q_{v 2}+2 \mu_{1} D_{0} q_{v 2}+\alpha_{1} q_{v 1}^{2}+3 \beta_{1} q_{v 0} q_{v 1}^{2} \\
& \quad+2 \alpha_{1} q_{v 0} q_{v 2}+3 \beta_{1} q_{v 0}^{2} q_{v 2}+\gamma_{1} q_{v 2} q_{w 0}^{2} \\
& \quad+2 \gamma_{1} q_{v 1} q_{w 0} q_{w 1}+\kappa_{1} q_{w 1}^{2}+\gamma_{1} q_{v 0} q_{w 1}^{2}+2 \kappa_{1} q_{w 0} q_{w 2} \\
& \quad+2 \gamma_{1} q_{v 0} q_{w 0} q_{w 2}=0, \\
& D_{0}^{2} q_{w 3}+\omega_{2}^{2} q_{w 3}+2 \mu_{2} D_{2} q_{w 0}+D_{1}^{2} q_{w 1}+2 \mu_{2} D_{1} q_{w 1} \\
& \quad+2 D_{0} D_{1} q_{w 2}+2 \mu_{2} D_{0} q_{w 2}+\alpha_{2} q_{v 2} q_{w 0}+\kappa_{2} q_{v 1}^{2} q_{w 0} \\
& \quad+3 \beta_{2} q_{w 0} q_{w 1}^{2}+\alpha_{2} q_{v 1} q_{w 1}+3 \beta_{2} q_{w 0}^{2} q_{w 2} \\
& \quad+2 \kappa_{2} q_{v 0} q_{v 2} q_{w 0}+2 \kappa_{2} q_{v 0} q_{v 1} q_{w 1}+\kappa_{2} q_{v 0}^{2} q_{w 0} \\
& \quad+\alpha_{2} q_{v 0} q_{w 2}=0 .
\end{aligned}
$$

By solving the set of equations in (40), we have

$$
\begin{aligned}
q_{v 0}= & A_{v}\left(T_{1}, T_{2}\right) \exp \left(i \omega_{1} T_{0}\right) \\
& +\bar{A}_{v}\left(T_{1}, T_{2}\right) \exp \left(-i \omega_{1} T_{0}\right),
\end{aligned}
$$

$$
\begin{aligned}
q_{w 0}= & A_{w}\left(T_{1}, T_{2}\right) \exp \left(i \omega_{2} T_{0}\right) \\
& +\bar{A}_{w}\left(T_{1}, T_{2}\right) \exp \left(-i \omega_{2} T_{0}\right)
\end{aligned}
$$

where $A_{v}$ and $A_{w}$ are unknown complex functions and $\bar{A}_{v}$ and $\bar{A}_{w}$ are the conjugates of $A_{v}$ and $A_{w}$, respectively. For ease of description, conjugate terms are denoted by $c c$. The governing equations for $A_{v}$ and $A_{w}$ can be solved by requiring $q_{v 0}, q_{v 1}, q_{w 0}$, and $q_{w 1}$ to be functions with a period of $T_{0}$.

By substituting (44) into (41), we have

$$
\begin{aligned}
& D_{0}^{2} q_{v 1}+\omega_{1}^{2} q_{v 1}=-2 i \omega_{1} D_{1} A_{v} e^{i 2 \omega_{1} T_{0}} \\
& -2 i \omega_{1} \mu_{1} A_{v} e^{i 2 \omega_{1} T_{0}} \\
& -\gamma_{1} A_{w}^{2} A_{v} e^{i\left(2 \omega_{2}+\omega_{1}\right) T_{0}} \\
& -\gamma_{1} \bar{A}_{w}^{2} A_{v} e^{i\left(\omega_{1}-2 \omega_{2}\right) T_{0}} \\
& -3 \beta_{1} A_{v}^{2} \bar{A}_{v} e^{i \omega_{1} T_{0}} \\
& -2 \gamma_{1} A_{w} \bar{A}_{w} A_{v} e^{i \omega_{1} T_{0}} \\
& -\beta_{1} A_{v}^{3} e^{i 3 \omega_{1} T_{0}}-\alpha_{1} A_{v}^{2} e^{i 2 \omega_{1} T_{0}} \\
& -2 \alpha_{1} A_{v} \bar{A}_{v}-2 \kappa_{1} A_{w} \bar{A}_{w} \\
& -\kappa_{1} A_{w}^{2} e^{i 2 \omega_{2} T_{0}}+c c, \\
& D_{0}^{2} q_{w 1}+\omega_{1}^{2} q_{w 1}=-2 i \omega_{2} D_{1} A_{w} e^{i 2 \omega_{2} T_{0}} \\
& -2 i \omega_{2} \mu_{2} A_{w} e^{i 2 \omega_{2} T_{0}} \\
& -3 \beta_{2} A_{w}^{2} \bar{A}_{w} e^{i \omega_{2} T_{0}} \\
& -2 \kappa_{2} A_{v} \bar{A}_{v} A_{w} e^{i \omega_{2} T_{0}} \\
& -\beta_{2} A_{w}^{3} e^{3 i \omega_{2} T_{0}} \\
& -\alpha_{2} A_{v} A_{w} e^{i\left(\omega_{1}+\omega_{2}\right) T_{0}} \\
& -\alpha_{2} A_{v} \bar{A}_{w} e^{i\left(\omega_{1}-\omega_{2}\right) T_{0}} \\
& -\kappa_{2} A_{v}^{2} A_{w} e^{i\left(2 \omega_{1}+\omega_{2}\right) T_{0}} \\
& -\kappa_{2} \bar{A}_{v}^{2} A_{w} e^{i\left(\omega_{2}-2 \omega_{1}\right) T_{0}}+f_{2} e^{i \Omega_{z} T_{0}} \\
& +c c \text {. }
\end{aligned}
$$

From (45), we can easily find that the terms that contain $e^{i 2 \omega_{1} T_{0}}$ and $e^{i 2 \omega_{2} T_{0}}$ can lead to secular terms in the solutions of the above equation. In addition, from (30), we know that the coupling of the in-plane and out-of-plane motion of the transmission line exhibits linearity, quadratic nonlinearity, and cubic nonlinearity. Thus, if parameters exist that allow the natural frequencies of the in-plane and out-of-plane vibration of the transmission line to satisfy any of the relations $\omega_{1} \approx \omega_{2}, \omega_{1} \approx 2 \omega_{2}$, and $\omega_{2} \approx 2 \omega_{1}$, the solutions to the 
abovementioned partial differential equations that describe the motion of the transmission line will contain an additional term that links $q_{v 1}$ and $q_{w 1}$, which is exactly the secular term caused by internal resonance. Therefore, when solving (45), we should consider the cases of $\omega_{1} \approx \omega_{2}, \omega_{1} \approx 2 \omega_{2}$, and $\omega_{2} \approx 2 \omega_{1}$. In view of the complexity of the problem-solving and the purpose of this study, we merely take the case of $\omega_{1} \approx \omega_{2}$ as an example to illustrate the analysis process.

Assume that the natural frequencies of the in-plane and out-of-plane vibrations of the transmission line meet the following relationship:

$$
\omega_{2}-\omega_{1}=\varepsilon \sigma, \quad \sigma=O(1) .
$$

Thus,

$$
\begin{aligned}
\left(\omega_{2}+\omega_{1}\right) T_{0} & =2 \omega_{1} T_{0}+\varepsilon \sigma T_{0}=2 \omega_{1} T_{0}+\sigma T_{1} \\
& =2 \omega_{2} T_{0}+\sigma T_{1}, \\
2 \omega_{2} T_{0} & =2\left(\omega_{1}+\varepsilon \sigma\right) T_{0}=2 \omega_{2} T_{0}+2 \sigma T_{1} .
\end{aligned}
$$

By substituting (47) into (45), we can obtain the requirements to eliminate the secular terms as follows:

$$
\begin{array}{r}
2 i \omega_{1}\left(D_{1} A_{v}+\mu_{1} A_{v}\right)+\alpha_{1} A_{v}^{2}+\kappa_{1} A_{w}^{2} e^{i 2 \sigma T_{1}}=0 \\
2 i \omega_{2}\left(D_{1} A_{w}+\mu_{2} A_{w}\right)+\alpha_{2} A_{v} A_{w} e^{i \sigma T_{1}}=0
\end{array}
$$

The following formulas are introduced:

$$
A_{r}=\frac{a_{r}}{2} e^{j \theta_{r}}, \quad r=v, w
$$

By substituting (49) into (48) and separating the real part from the imaginary part, we have

$$
\begin{aligned}
& D_{1} a_{v}+\mu_{1} a_{v}+\frac{\alpha_{1} a_{v}^{2}}{2 \omega_{1}} \sin \theta_{v} \\
& \quad+\frac{\kappa_{1} a_{w}^{2}}{4 \omega_{1}} \sin 2\left(\theta_{w}+\sigma T_{1}\right) \sec \theta_{v}=0, \\
& a_{v} D_{1} \theta_{v} \\
& \quad-\frac{\csc \theta_{v}}{4 \omega_{1}}\left[\alpha_{1} a_{v}^{2} \cos 2 \theta_{v}+\kappa_{1} a_{w}^{2} \cos 2\left(\theta_{w}+\sigma T_{1}\right)\right] \\
& \quad=0, \\
& D_{1} a_{w}+\mu_{2} a_{w}+\frac{\alpha_{2} a_{v} a_{w}}{4 \omega_{2} \cos \theta_{w}} \sin \left(\theta_{v}+\theta_{w}+\sigma T_{1}\right)=0, \\
& a_{w} D_{1} \theta_{w}+\frac{\alpha_{2} a_{v} a_{w}}{4 \omega_{2} \cos \theta_{w}} \cos \left(\theta_{v}+\theta_{w}+\sigma T_{1}\right)=0 .
\end{aligned}
$$

Let us introduce the new phase angle as follows:

$$
\begin{aligned}
& \varphi_{1}=2\left(\theta_{w}+\sigma T_{1}\right), \\
& \varphi_{2}=\theta_{v}+\theta_{w}+\sigma T_{1} .
\end{aligned}
$$

By substituting (51) into (50), we have

$$
\begin{aligned}
D_{1} a_{v}+\mu_{1} a_{v}+\frac{\alpha_{1} a_{v}^{2}}{2 \omega_{1}} \sin \theta_{v}+\frac{\kappa_{1} a_{w}^{2}}{4 \omega_{1}} \sin \varphi_{1} \sec \theta_{v}=0 \\
a_{v} D_{1} \theta_{v}-\frac{\csc \theta_{v}}{4 \omega_{1}}\left[\alpha_{1} a_{v}^{2} \cos 2 \theta_{v}+\kappa_{1} a_{w}^{2} \cos \varphi_{1}\right]=0 \\
D_{1} a_{w}+\mu_{2} a_{w}+\frac{\alpha_{2} a_{v} a_{w}}{4 \omega_{2} \cos \theta_{w}} \sin \varphi_{2}=0 \\
a_{w} D_{1} \theta_{w}+\frac{\alpha_{2} a_{v} a_{w}}{4 \omega_{2} \cos \theta_{w}} \cos \varphi_{2}=0
\end{aligned}
$$

When solving (52), we must discuss whether the damping of the system is considered. In addition, even if we can obtain the one-order approximate steady-state solution of the equation, we must investigate its asymptotic stability to determine whether it actually exists. Hence, we can see that the above issue is so complicated that it should be specifically studied. Due to the limitation of the abovementioned approximation method, it is difficult to obtain the theoretical solutions to the equations of coupled in-plane and out-of-plane motion for transmission lines. Therefore, in the subsequent section, we intend to perform the quantitative analysis of (30) by using numerical methods.

Because the main purpose of this article is to reveal the inherently nonlinear vibration characteristics of transmission lines, the above MS analysis is merely used to determine the requirements for eliminating secular terms in the solutions of the partial differential equations of transmission lines. Because the second-order approximation solutions can provide enough information for most practical problems, it is usually necessary to give only the second-order approximation solutions of a nonlinear system by using the MS method [27-29]. For this reason, we consider only the power of $\varepsilon$ up to 2 in this paper. From the requirements for eliminating secular terms, it is clear that internal resonance is considered to exist in the transmission line if parameters exist that allow the natural frequencies of the in-plane and out-ofplane vibration of the transmission line to satisfy any of the following relations: $\omega_{1} \approx \omega_{2}, \omega_{1} \approx 2 \omega_{2}$, and $\omega_{2} \approx 2 \omega_{1}$.

\section{Numerical Analysis of the Characteristics of the Nonlinear Coupled In-Plane and Out-of-Plane Internal Resonance of Transmission Lines}

In numerical analysis, the Runge-Kutta method is widely used to obtain the approximate solutions of differential equations. This method is so accurate that most computer packages designed to find numerical solutions for differential equations will use it by default-the fourth-order RungeKutta method. Therefore, we employ a higher-order RungeKutta method to obtain the numerical solutions to these equations, with the aim of revealing the nonlinear vibration characteristics of transmission lines. 


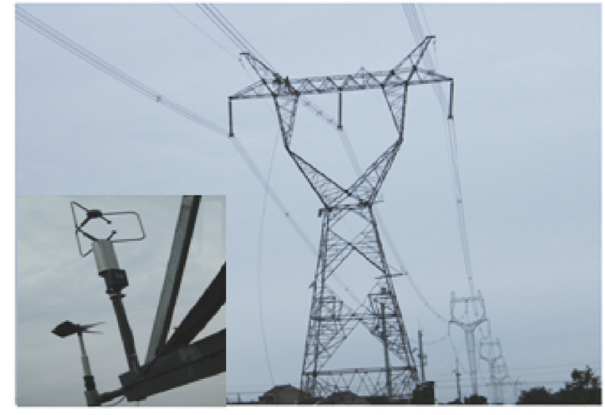

FIGURE 2: A high-voltage transmission line.

The following state variables are introduced:

$$
\begin{aligned}
& Y_{1}=q_{v}, \\
& Y_{2}=\dot{q}_{v}, \\
& Y_{3}=q_{w}, \\
& \dot{Y}_{4}=\dot{q}_{w} .
\end{aligned}
$$

Thus, (30) can be transformed to state functions:

$$
\begin{aligned}
& \dot{Y}_{1}=Y_{2}, \\
& \dot{Y}_{2} \\
& =\frac{\left(P_{v}-a_{2} Y_{1}-a_{3} Y_{1}^{2}-a_{4} Y_{3}^{2}-a_{5} Y_{1}^{3}-a_{6} Y_{3}^{2} Y_{1}-a_{7} Y_{2}\right)}{a_{1}}, \\
& \dot{Y}_{3}=Y_{4}, \\
& \dot{Y}_{4}=\frac{\left(P_{w}-b_{2} Y_{3}-b_{3} Y_{1} Y_{3}-b_{4} Y_{1}^{2} Y_{3}-b_{5} Y_{3}^{3}-b_{6} Y_{4}\right)}{b_{1}} .
\end{aligned}
$$

4.1. Calculation of the Parameters. A relatively large singlespan transmission line in a high-voltage transmission line system in East China (Figure 2) is selected as an example for analysis [30]. The conductors of this span of the transmission line are composed of LGJ-630/45 ASCR cables. Table 1 lists the design parameters of the selected transmission line. The maximum design wind speed at a height of $10 \mathrm{~m}$ above the ground for safe operation of the selected transmission line is $25.3 \mathrm{~m} / \mathrm{s}$. In the actual transmission line, the conductors of each phase are quad-bundled conductors. To simplify calculation, in the finite element (FE) modeling process, the quad-bundled conductors of each phase are equivalently simplified to one conductor based on the following principles: the bundled conductors have the same windward area, the same total operational tension, and the same linear density. Table 2 lists the parameters of the transmission line after simplification.

The sag-to-span ratio $d_{c} / L_{c}$ of the selected transmission line is calculated based on the parameters listed in Table 1 to be approximately $1 / 29$, which is far smaller than $1 / 8$. Thus, (30) can be used to analyze the nonlinear vibration characteristics of this transmission line.
4.2. Modal Analysis of the Transmission Line. Based on the actual characteristics of the selected transmission line in combination with the design parameters listed in Tables 1 and 2, LINK10 elements in ANSYS are used to construct an FE model for the selected transmission line. First, a modal analysis is performed to determine the natural frequency of vibration of each order of the transmission line model; this provides a basis for the subsequent numerical simulation. To validate the FE model constructed for a single transmission line, in this section, the FE simulation results with respect to the natural vibration frequencies of the transmission line are also compared with the theoretical solutions.

A transmission line is a typical type of suspended cable structure, and the analytical solutions for the natural frequencies of its vibration can be obtained based on the singlecable theory. A transmission line resists external loading primarily by self-stretching and consequently will undergo relatively large displacement under loading. Therefore, the geometric nonlinearity of a transmission line must be taken into consideration in the calculation. When constructing an FE model of a transmission line, one crucial point is to determine the initial configuration of the cable. Specifically, on the one hand, this means to determine the spatial location of the transmission line by determining the equilibrium and deformation compatibility equations for the transmission line based on the existing suspended cable theory; on the other hand, this means to transition the transmission line from its initial stress-free state to an initial loaded state based on a certain configuration-seeking approach.

The catenary and parabolic methods are the main methods used to determine the equilibrium equation for a suspended cable. The former is an accurate method, whereas the latter is an approximate method. However, when the sag-to-span ratio $d_{c} / L_{c}$ of a cable is less than $1 / 8$, the parabolic method can also yield a relatively accurate solution for the initial configuration of the transmission line. Here, assuming that the vertical load on a transmission line is evenly distributed along the span, as shown in Figure 3, the equation for the spatial configuration of the transmission line is

$$
x(y)=\frac{q_{c}}{2 T_{c 0}} y\left(L_{c}-y\right)+\frac{\Delta_{c}}{L_{c}} y,
$$

where $q_{c}$ represents the vertical load on the transmission line, which in this case is the dead weight of the transmission line; $T_{c 0}$ represents the initial horizontal tension in the transmission line $\left(T_{c 0}=\left(q_{c} L_{c}^{2}\right) /\left(8 d_{c}\right)\right) ; L_{c}$ represents the horizontal span of the transmission line; $\Delta_{c}$ represents the difference in height between the two ends of the transmission line; and $d_{c}$ represents the mid-span sag of the transmission line.

Considering that a transmission line generally has a small sag, the first-order natural frequency corresponding to the out-of-plane vibration of the transmission line is the smallest frequency of all the frequencies of its vibration. According to the linear vibration theory of suspended cables, for a horizontal suspended cable with two ends hinged at the same height and whose mass is evenly distributed, the natural 


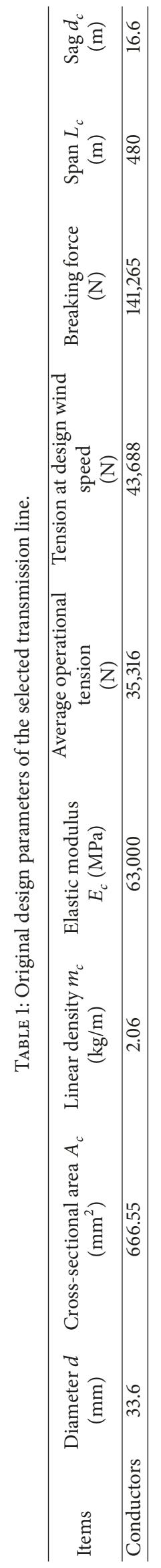


TABLE 2: Design parameters of the selected transmission line after simplification.

\begin{tabular}{lccccc}
\hline Items & $\begin{array}{c}\text { Diameter } d \\
(\mathrm{~mm})\end{array}$ & $\begin{array}{c}\text { Density } \rho_{c} \\
\left(\mathrm{~kg} / \mathrm{m}^{3}\right)\end{array}$ & $\begin{array}{c}\text { Linear density } m_{c} \\
(\mathrm{~kg} / \mathrm{m})\end{array}$ & $\begin{array}{c}\text { Linear load } q_{c} \\
(\mathrm{~N} / \mathrm{m})\end{array}$ & $\begin{array}{c}\text { Initial strain } \\
\varepsilon_{c 0}\end{array}$ \\
\hline Conductors & 134.4 & $3,090.5$ & 8.24 & 80.752 & 0.00084 \\
\hline
\end{tabular}

frequencies of vibration and the corresponding modes under the action of gravity alone are as follows:

(a) The out-of-plane swing and the in-plane vibration of the suspended cable are not coupled. Thus, the natural circular frequencies of the out-of-plane swing and the corresponding modes can be expressed as follows:

$$
\begin{gathered}
\omega_{w}=\frac{n \pi}{L_{c}} \sqrt{\frac{T_{c 0}}{m_{c}}}, \quad n=1,2,3, \ldots, \\
\Phi_{w}=A_{n} \sin \frac{n \pi y}{L_{c}}, \quad n=1,2,3, \ldots,
\end{gathered}
$$

where $\omega_{w}$ represents the natural circular frequency of the out-of-plane vibration of the suspended cable, $n$ represents the modal number, $m_{c}$ represents the mass of the suspended cable per unit length, $\Phi_{w}$ represents the out-of-plane mode of vibration of the suspended cable, and $A_{n}$ represents the modal amplitude (each of the other symbols has the same meaning as previously described).

(b) The in-plane antisymmetric vibration of the suspended cable will not generate an increment in its horizontal dynamic tension. The natural circular frequencies of its vibration and the corresponding modes can be expressed as follows:

$$
\begin{gathered}
\omega_{v}=\frac{2 n \pi}{L_{c}} \sqrt{\frac{T_{c 0}}{m_{c}}}, \quad n=1,2,3, \ldots, \\
\Phi_{v}=B_{n} \sin \frac{2 n \pi y}{L_{c}}, \quad n=1,2,3, \ldots,
\end{gathered}
$$

where $\omega_{v}$ represents the natural circular frequency of the in-plane antisymmetric vibration of the suspended cable, $n$ represents the modal number, $\Phi_{v}$ represents the mode of the in-plane antisymmetric vibration of the suspended cable, and $B_{n}$ represents the modal amplitude (each of the other symbols has the same meaning as previously described).

(c) The in-plane symmetric vibration of the suspended cable will generate an additional increment in its dynamic tension. The natural circular frequencies of its vibration and the corresponding modes can be determined by solving the following transcendental equations:

$$
\begin{aligned}
\tan \frac{\omega}{2} & =\frac{\omega}{2}-\frac{4}{\lambda^{2}}\left(\frac{\Phi}{2}\right)^{3}, \\
\omega & =\frac{\omega_{\text {vant }} L_{c}}{\sqrt{T_{c 0} / m_{c}}},
\end{aligned}
$$

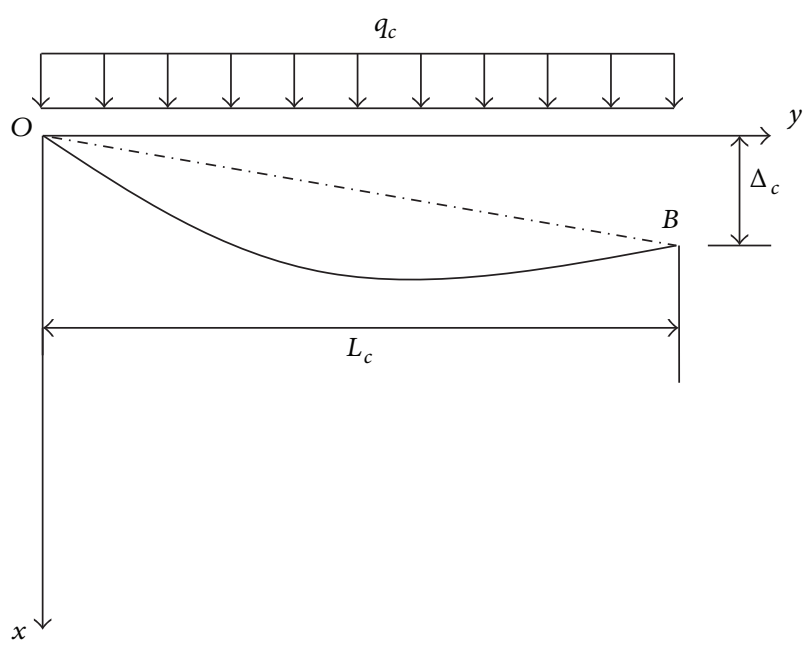

Figure 3: A schematic diagram of the calculation for a single cable when the load is evenly distributed along the span.

$$
\begin{aligned}
\lambda^{2} & =\frac{\left(m_{c} g L_{c} / T_{c 0}\right)^{2} L_{c}}{T_{c 0} L_{c} /\left(E_{c} A_{c}\right)}, \\
\Phi_{\text {vant }} & =\frac{\tilde{h}}{T_{c 0} \omega^{2}}\left(1-\tan \frac{\omega}{2} \sin \frac{\omega y}{L_{c}}-\cos \frac{\omega y}{L_{c}}\right),
\end{aligned}
$$

where $\omega_{\text {vant }}$ represents the natural circular frequency of the in-plane symmetric vibration of the suspended cable, $\Phi_{\text {vant }}$ represents the in-plane symmetric mode of vibration of the suspended cable, $E_{c}$ represents the elastic modulus of the suspended cable, $A_{c}$ represents the cross-sectional area of the suspended cable, and $\widetilde{h}$ represents the additional tension in the suspended cable (each of the other symbols has the same meaning as previously described).

By substituting the parameters listed in Tables 1 and 2 into (55)-(58), the theoretical solutions for the natural vibration frequencies of the first eight orders of the transmission line in the three directions can be obtained (they are shown in Table 3). Table 4 summarizes the linear natural vibration frequencies of the transmission line obtained from FE simulation.

A comparison of Tables 3 and 4 shows that the natural vibration frequencies of the transmission line model obtained from FE simulation are in relatively good agreement with the theoretical values, with the largest difference being approximately $1 \%$. This indicates that the transmission line model constructed in this work is relatively reliable. Moreover, Tables 3 and 4 also demonstrate that the following relationships between the natural frequencies of the in-plane and out-of-plane modes of vibration of the transmission line 
TABLE 3: Theoretical linear natural vibration frequencies of the transmission line model.

\begin{tabular}{|c|c|c|c|c|c|c|c|c|c|}
\hline & $\begin{array}{c}\text { Calculation } \\
\text { equation }\end{array}$ & $\begin{array}{l}\text { First } \\
\text { order }\end{array}$ & $\begin{array}{c}\text { Second } \\
\text { order }\end{array}$ & $\begin{array}{l}\text { Third } \\
\text { order }\end{array}$ & $\begin{array}{c}\text { Fourth } \\
\text { order }\end{array}$ & Fifth order & Sixth order & $\begin{array}{c}\text { Seventh } \\
\text { order }\end{array}$ & $\begin{array}{l}\text { Eighth } \\
\text { order }\end{array}$ \\
\hline $\begin{array}{l}\text { Out-of-plane vibration } \\
\text { frequency } f_{w}(\mathrm{~Hz})\end{array}$ & $f_{w}=\frac{\omega_{w}}{(2 \pi)}$ & 0.136 & 0.273 & 0.409 & 0.546 & 0.682 & 0.818 & 0.955 & 1.091 \\
\hline $\begin{array}{l}\text { In-plane symmetric vibration } \\
\text { frequency } f_{v}(\mathrm{~Hz})\end{array}$ & $f_{v}=\frac{\omega_{v}}{(2 \pi)}$ & 0.344 & 0.46 & - & - & - & - & - & - \\
\hline $\begin{array}{l}\text { In-plane antisymmetric } \\
\text { vibration frequency } f_{\text {vant }}(\mathrm{Hz})\end{array}$ & $f_{\text {vant }}=\frac{\omega_{\text {vant }}}{(2 \pi)}$ & 0.273 & 0.546 & 0.818 & 1.091 & 1.364 & 1.637 & 1.91 & 2.182 \\
\hline
\end{tabular}

TABLE 4: Natural vibration frequencies of the transmission line model obtained from FE simulation.

\begin{tabular}{lccccccccc}
\hline & $\begin{array}{c}\text { Calculation } \\
\text { equation }\end{array}$ & $\begin{array}{c}\text { First } \\
\text { order }\end{array}$ & $\begin{array}{c}\text { Second } \\
\text { order }\end{array}$ & $\begin{array}{c}\text { Third } \\
\text { order }\end{array}$ & $\begin{array}{c}\text { Fourth } \\
\text { order }\end{array}$ & Fifth order Sixth order & $\begin{array}{c}\text { Seventh } \\
\text { order }\end{array}$ & $\begin{array}{c}\text { Eighth } \\
\text { order }\end{array}$ \\
\hline $\begin{array}{l}\text { Out-of-plane vibration } \\
\text { frequency } f_{w}(\mathrm{~Hz})\end{array}$ & $f_{w}=\frac{\omega_{w}}{(2 \pi)}$ & 0.136 & 0.272 & 0.408 & 0.544 & 0.68 & 0.816 & 0.952 & 1.088 \\
$\begin{array}{l}\text { In-plane symmetric vibration } \\
\text { frequency } f_{v}(\mathrm{~Hz})\end{array}$ & $f_{v}=\frac{\omega_{v}}{(2 \pi)}$ & 0.346 & 0.46 & 0.686 & 0.954 & 1.225 & 1.497 & 1.769 & 2.042 \\
$\begin{array}{l}\text { In-plane antisymmetric } \\
\text { vibration frequency } f_{\text {vant }}(\mathrm{Hz})\end{array}$ & $f_{\text {vant }}=\frac{\omega_{\text {vant }}}{(2 \pi)}$ & 0.27 & 0.544 & 0.816 & 1.088 & 1.36 & 1.633 & 1.91 & 2.18 \\
\hline
\end{tabular}

always exist: $\omega_{v} \approx \omega_{w}, \omega_{v} \approx 2 \omega_{w}$, and $\omega_{v} \approx 3 \omega_{w}$. In other words, within the scope of the linear theory, the natural vibration frequencies of the transmission line are always multiples of one another.

Figure 4 shows the modes of vibration of the first nine orders obtained from FE simulation. As demonstrated in Figure 4, of all the modes of vibration of the transmission line, the first-order out-of-plane vibration is the most easily excitable. The higher-order frequencies of in-plane vibration are approximately integer multiples of the fundamental frequency, and this relationship also exists between the frequencies of in-plane antisymmetric vibration and the fundamental frequency. In addition to the relationship to the fundamental frequency, the higher-order frequencies of the out-of-plane vibration are also approximately equal to those of the inplane vibration, and the in-plane and out-of-plane modes of vibration corresponding to the same frequency are also similar to one another.

4.3. Numerical Analysis of the Nonlinear Internal Resonance of the Transmission Line When in Free Vibration. When only the free vibration of the transmission line under the action of the initial displacement is considered, in (54), $P_{v}=0$ and $P_{w}=0$.

The structural damping of a transmission line is often relatively small, and it can generally be set to 0.0005 . In addition, the effects of the structural damping of a transmission line on its vibration, particularly under strong wind conditions, are relatively insignificant, whereas aerodynamic damping relatively significantly affects the vibration of a transmission line. By comprehensively considering the aforementioned factors, in this study, when analyzing the free vibration of the transmission line, the damping $c_{c}$ of its in-plane and out-ofplane vibration is set to 0.001 . In the following sections, the effects of the initial potential energy of the transmission line on its internal resonance are analyzed in two scenarios.
4.3.1. When the Initial Potential Energy of the Transmission Line Is Relatively Low (i.e., When the Initial Mid-Span Displacement of the Transmission Line Is Relatively Small). Let us set the initial out-of-plane mid-span displacement and the initial velocity of out-of-plane motion of the transmission line to $1 \mathrm{~m}$ and $0.00005 \mathrm{~m} / \mathrm{s}$, respectively, and both its initial inplane mid-span displacement and the initial velocity of its inplane motion to 0 ; that is, the initial conditions described in (53) are set as follows: $q_{v}=0, \dot{q}_{v}=0, q_{w}=1, \dot{q}_{w}=0.00005$. The displacements of the transmission line obtained from numerical simulation are then divided by the horizontal span $L_{c}$ to obtain dimensionless displacements. The dimensionless velocities of in-plane and out-of-plane can also be obtained from the numerical simulation results of the in-plane and out-of-plane velocities multiplied by the first-order out-ofplane vibration period $T_{0}$ and then divided by the horizontal span $L_{c}$. The results are presented in Figures 5-7.

As demonstrated in Figure 5, when the initial energy of the transmission line is relatively low, its in-plane vibration displacement is relatively small, with the maximum amplitude being approximately $1 / 10$ that of its out-of-plane vibration displacement; in addition, the "beat vibration" phenomenon is inconspicuous in the response time history. As demonstrated in the Fourier spectra of the displacement response of the transmission line (Figure 6), the first-order mode of vibration is the primary out-of-plane mode of vibration. The out-of-plane vibration of the transmission line gives rise to the first-order in-plane antisymmetric mode of vibration and the first-order symmetric mode of vibration. In addition, the first-order in-plane symmetric vibration energy is also greater than the first-order in-plane antisymmetric vibration energy, and the in-plane vibration energy amplitude is smaller than the out-of-plane vibration energy amplitude by one order of magnitude. This indicates that the transmission line is inherently capable of generating nonlinear internal resonance and can undergo internal resonance even 


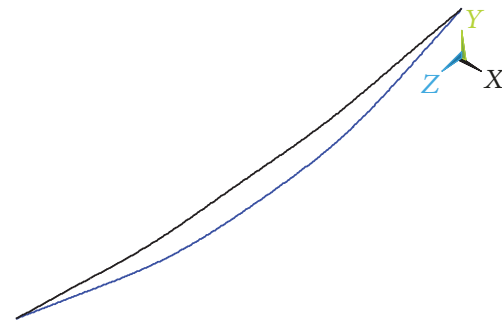

First out-of-plane mode of vibration (symmetric)

(a) First order, $0.136 \mathrm{~Hz}$

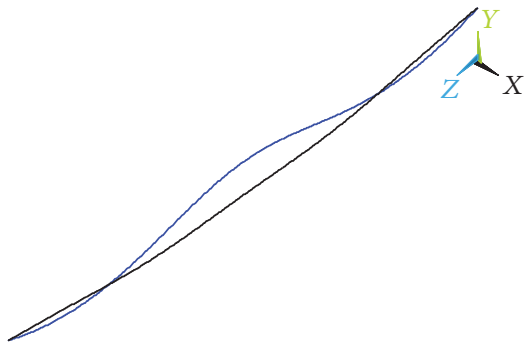

First in-plane mode of vibration (symmetric)

(d) Fourth order, $0.346 \mathrm{~Hz}$

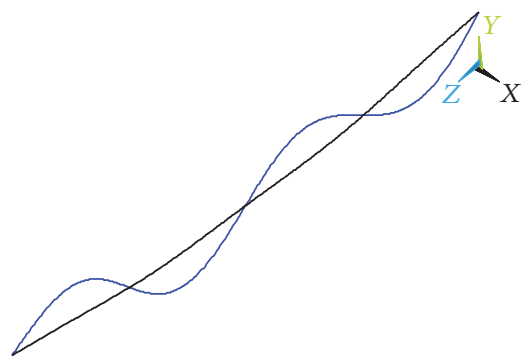

Second in-plane mode of vibration (antisymmetric)

(g) Seventh order, $0.544 \mathrm{~Hz}$

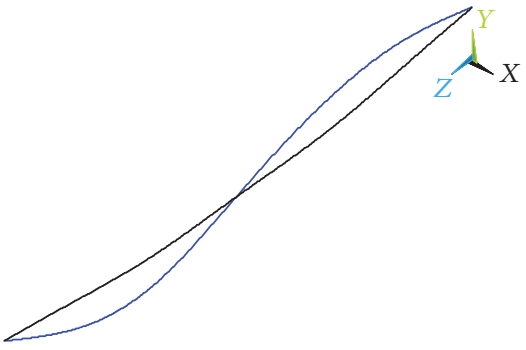

First in-plane mode of vibration (antisymmetric)

(b) Second order, $0.27 \mathrm{~Hz}$

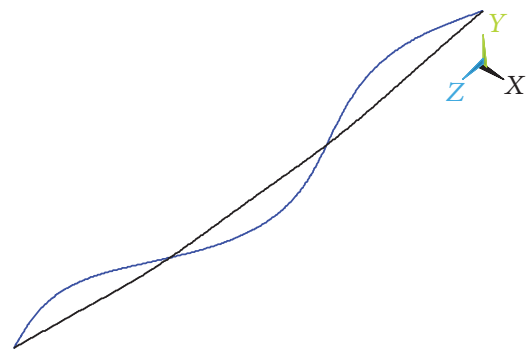

Third out-of-plane mode of vibration (symmetric)

(e) Fifth order, $0.408 \mathrm{~Hz}$

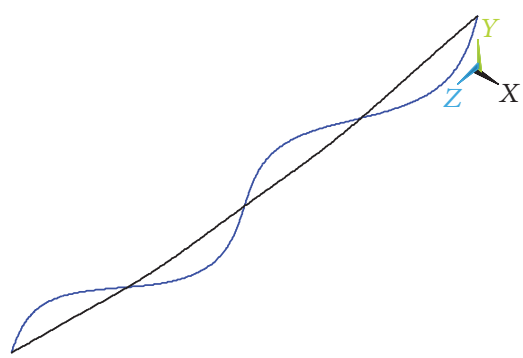

Fourth out-of-plane mode of vibration (antisymmetric)

(h) Eighth order, $0.544 \mathrm{~Hz}$

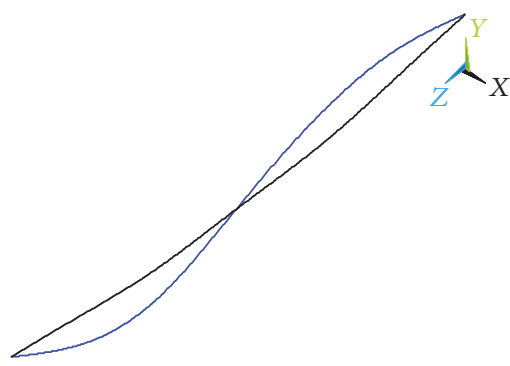

Second out-of-plane mode of vibration (antisymmetric)

(c) Third order, $0.272 \mathrm{~Hz}$

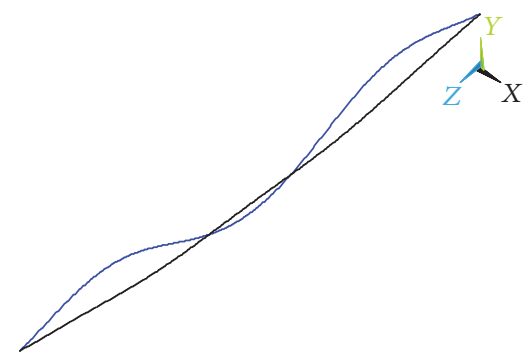

Second in-plane mode of vibration (symmetric)

(f) Sixth order, $0.46 \mathrm{~Hz}$

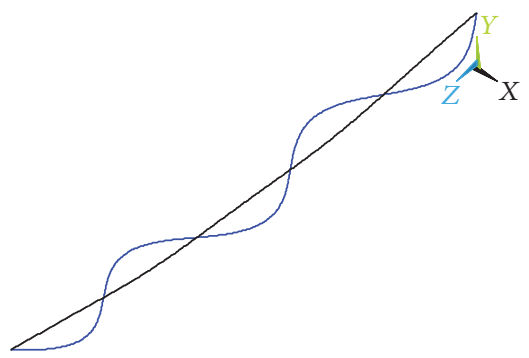

Fifth out-of-plane mode of vibration (symmetric)

(i) Ninth order, $0.68 \mathrm{~Hz}$

FIGURE 4: Modes of vibration of the transmission line of the first nine orders obtained from FE simulation.

when its initial energy is relatively low, but energy transfer and mode coupling are quite weak under this condition. As demonstrated in Figure 7, the out-of-plane and in-plane modes of vibration of the transmission line both exhibit periodic motion characteristics.

4.3.2. When the Initial Potential Energy of the Transmission Line Is Relatively High (i.e., When the Initial Mid-Span Displacement of the Transmission Line Is Relatively Large). Let us set the initial out-of-plane mid-span displacement of the transmission line to a relatively large value $(10 \mathrm{~m})$, the initial velocity of its out-of-plane mid-span motion to $0.00005 \mathrm{~m} / \mathrm{s}$, and both its initial in-plane mid-span displacement and the initial velocity of its in-plane motion to 0 ; that is, the initial conditions described in (53) are set as follows: $q_{v}=0, \dot{q}_{v}=0$, $q_{w}=10, \dot{q}_{w}=0.00005$. Figures 8-10 show the numerical simulation results.

As demonstrated in Figure 8, when the initial out-ofplane displacement of the transmission line is relatively large, as a result of the internal resonance the system energy constantly transfers between the modes that are in the $\omega_{v} \approx$ $\omega_{w}, \omega_{v} \approx 2 \omega_{w}$, and $\omega_{v} \approx 3 \omega_{w}$ relationships; in addition, the system energy also transfers between degrees of freedom that are similarly related to multiples of the frequency. On the one hand, the out-of-plane vibration energy transfers to the inplane direction, resulting in vibration with a relatively large amplitude. Figure 8 also demonstrates that the order of magnitude of the in-plane vibration displacement amplitude is commensurate with the out-of-plane vibration displacement amplitude; a phenomenon similar to "beat vibration" is also observed in the displacement response time history; that is, 


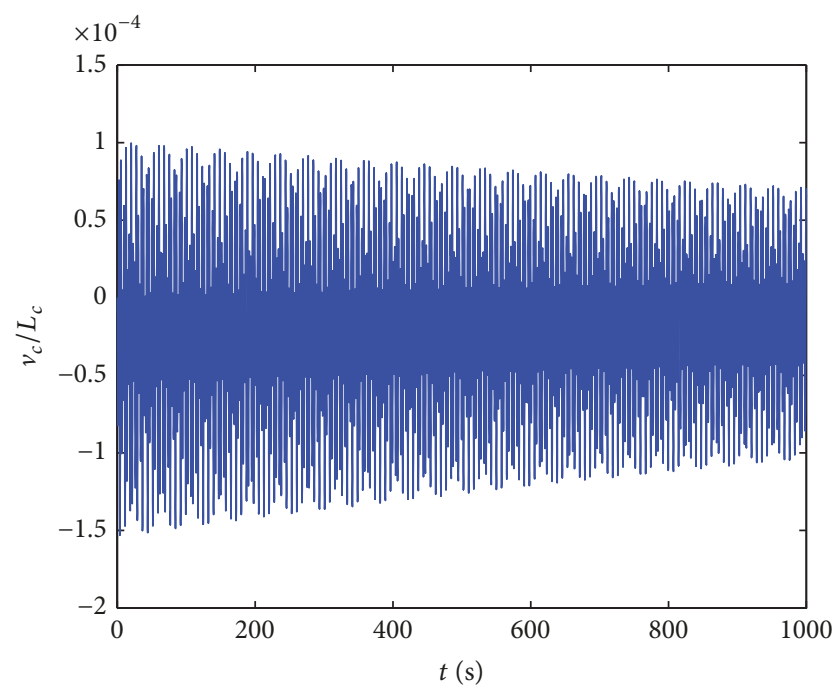

(a) In-plane mid-span displacement time history



(b) Out-of-plane mid-span displacement time history

FIGURE 5: In-plane and out-of-plane mid-span displacement time histories of the transmission line when its initial potential energy is relatively low.

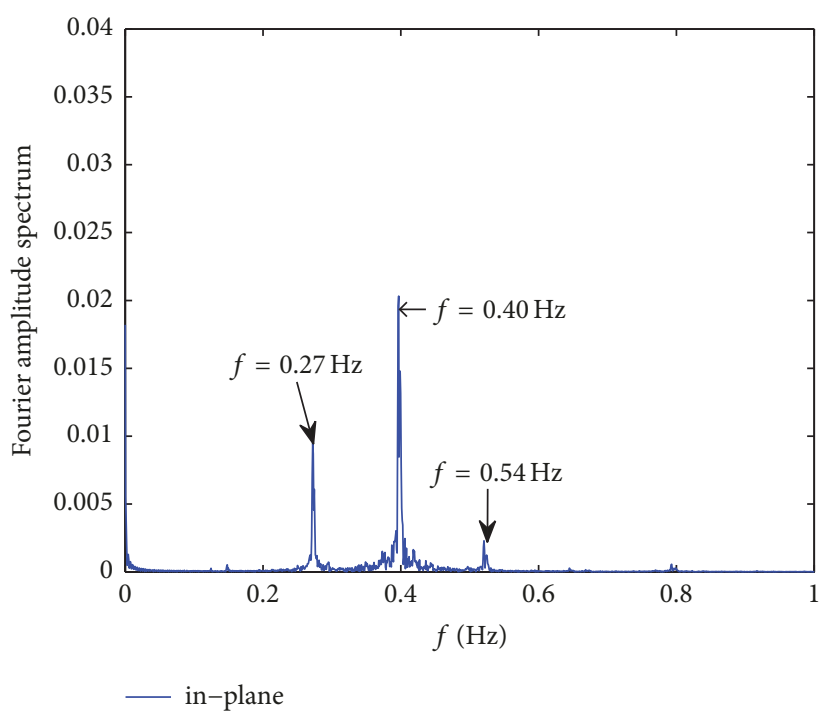

(a) In-plane

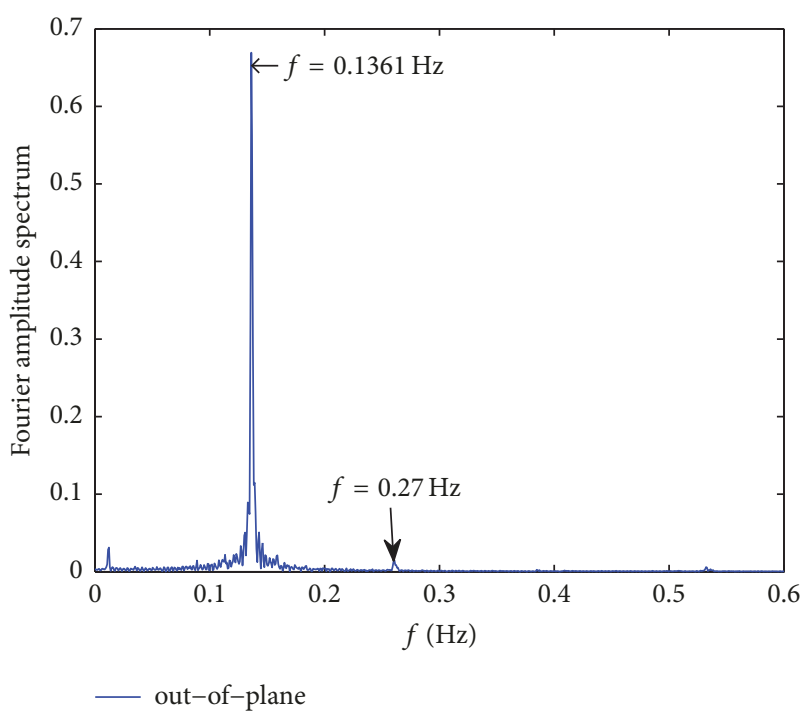

(b) Out-of-plane

FIGURE 6: Fourier spectra of the in-plane and out-of-plane mid-span displacements of the transmission line when its initial potential energy is relatively low.

as the out-of-plane vibration response gradually decreases, the in-plane vibration response at the corresponding time gradually increases and vice versa. Furthermore, from Figures 5 and 8 , we can also see that both of the maximum inplane and out-of-plane vibration amplitudes decrease with time. This is principally because the damping dissipates the free vibration energy. On the other hand, when Galerkin's method is employed in numerical simulation to perform first-order modal truncation on the vibration response of the transmission line to simplify the analytical process, the simulation results show that the second-order out-of-plane mode of vibration of the transmission line, as well as its firstorder symmetric mode of vibration and its first- to fourthorder antisymmetric modes of vibration, are still excited. As a result of the internal resonance, the first-order out-ofplane mode of vibration, which has relatively high energy, gives rise to the second-order out-of-plane mode of vibration, whose frequency is twice its own frequency. Because the natural frequencies of the second-order out-of-plane mode of vibration and the first-order in-plane antisymmetric mode 


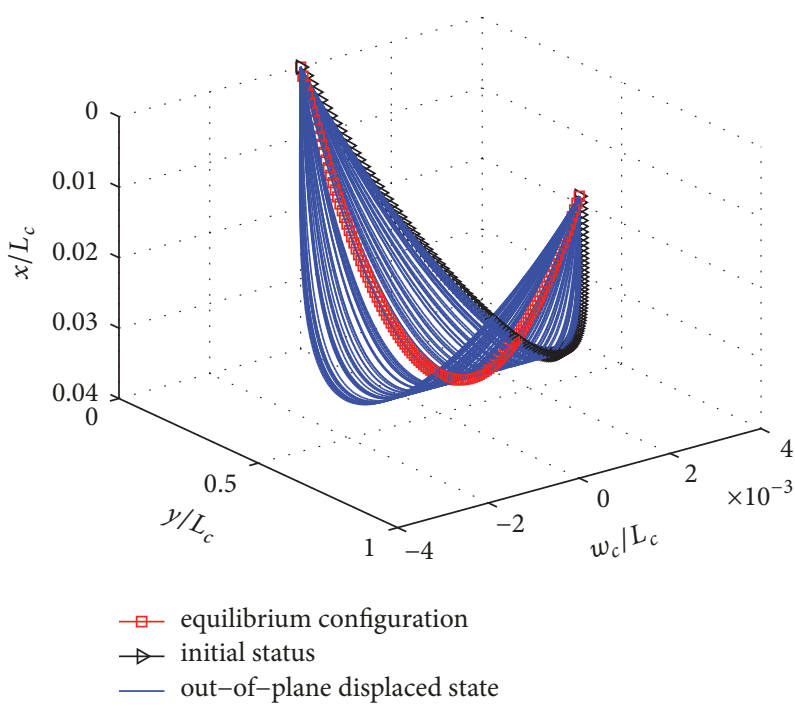

(a) Configuration of out-of-plane motion at various times

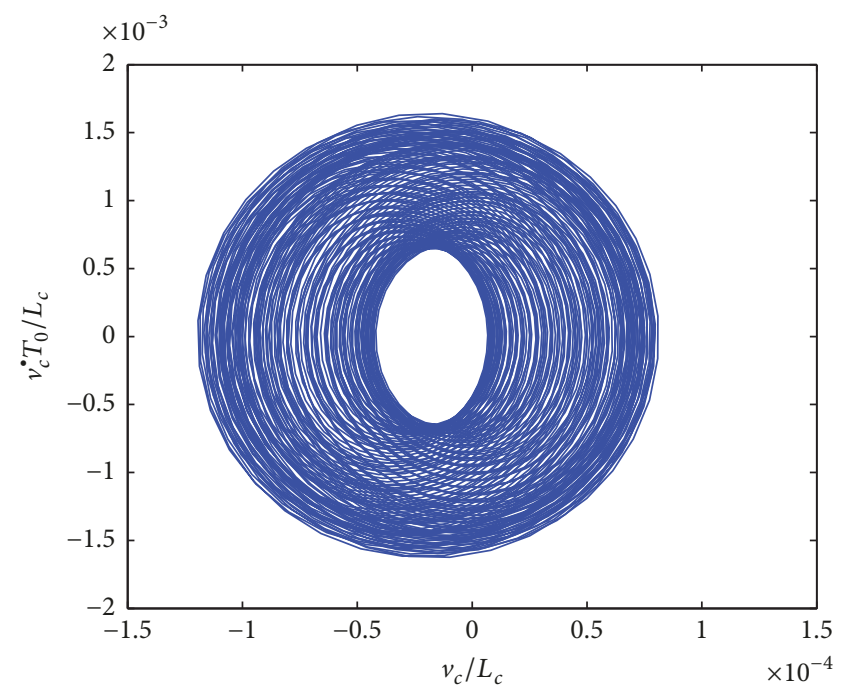

(c) In-plane mid-span phase trajectory

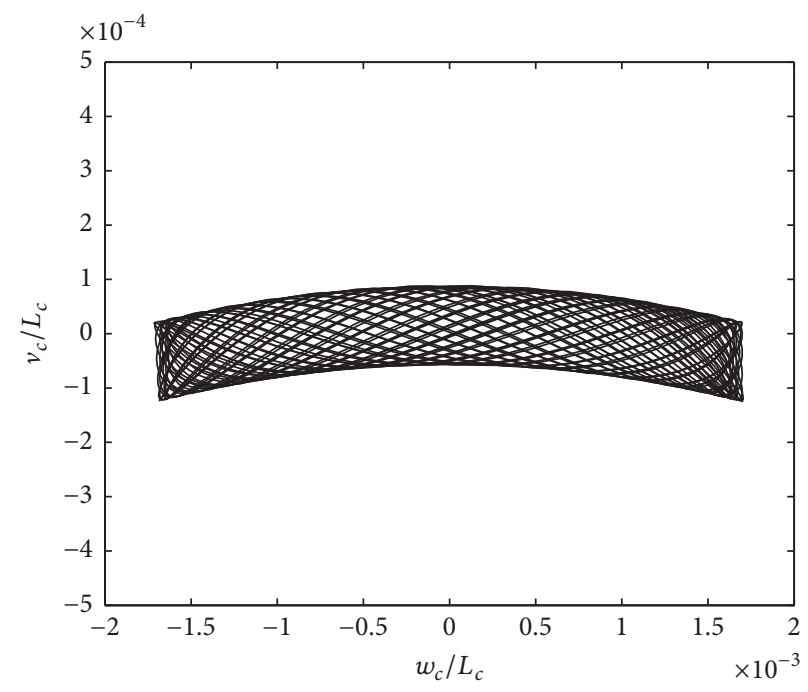

(b) In-plane and out-of-plane displacement responses in the phase space

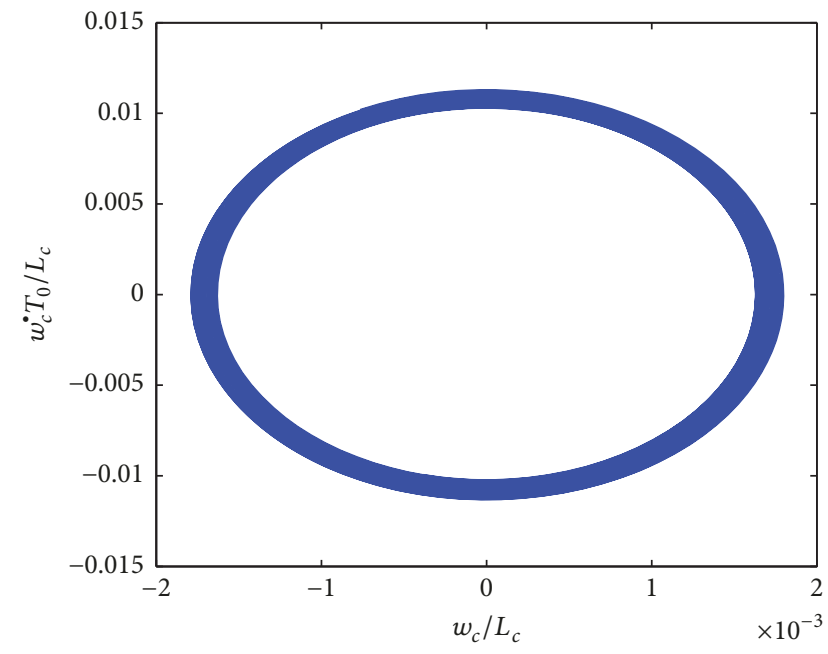

(d) Out-of-plane mid-span phase trajectory

FIGURE 7: Configuration of the out-of-plane motion and the phase-space response of the transmission line when its initial potential energy is relatively low.

of vibration are closely spaced, the out-of-plane vibration energy gradually transfers to the in-plane direction. In addition, because the natural frequency of the in-plane vibration of each order is two or three times the in-plane fundamental frequency, the energy also transfers between different inplane modes of vibration. The Fourier spectra of the displacement response shown in Figure 9 clearly demonstrate that the in-plane vibration also contains the first-order outof-plane mode of vibration. Similarly, as demonstrated in Figure 10, the in-plane motion of the transmission line is strongly coupled to its out-of-plane motion.

The aforementioned analysis shows that the out-of-plane vibration energy of the transmission line can give rise not only to in-plane antisymmetric vibration but also to first-order in-plane symmetric vibration, whose energy is the highest of all the in-plane modes of vibration. According to the suspended cable theory, in-plane symmetric vibration is the primary factor that causes changes in the dynamic tension in a suspended cable. To further illustrate the effects of internal resonance on the dynamic tension in the transmission line, the dynamic tension in the transmission line is calculated under the aforementioned two initial potential energy conditions (see Figure 11 for the results). In Figure 11, the tension is a dimensionless value obtained by dividing the dynamic tension $T_{c}$ obtained from simulation by the initial tension $T_{c 0}$. As demonstrated in Figure 11, when the transmission line vibrates with relatively low initial potential energy, the dynamic tension in the line is always positive, indicating that the transmission line is under tension. Meanwhile, when the line is in vibration, the dynamic tension in the transmission 


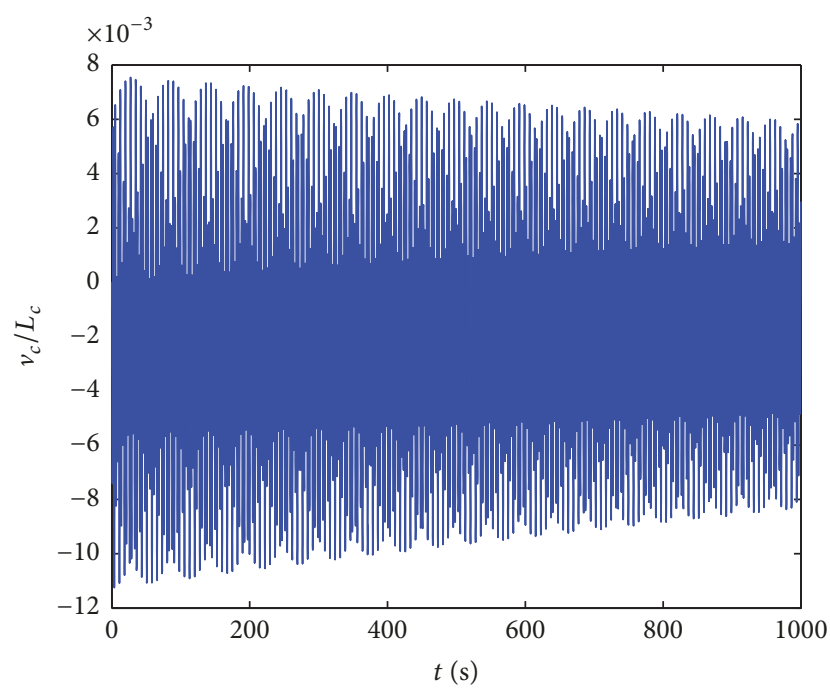

(a) In-plane mid-span displacement

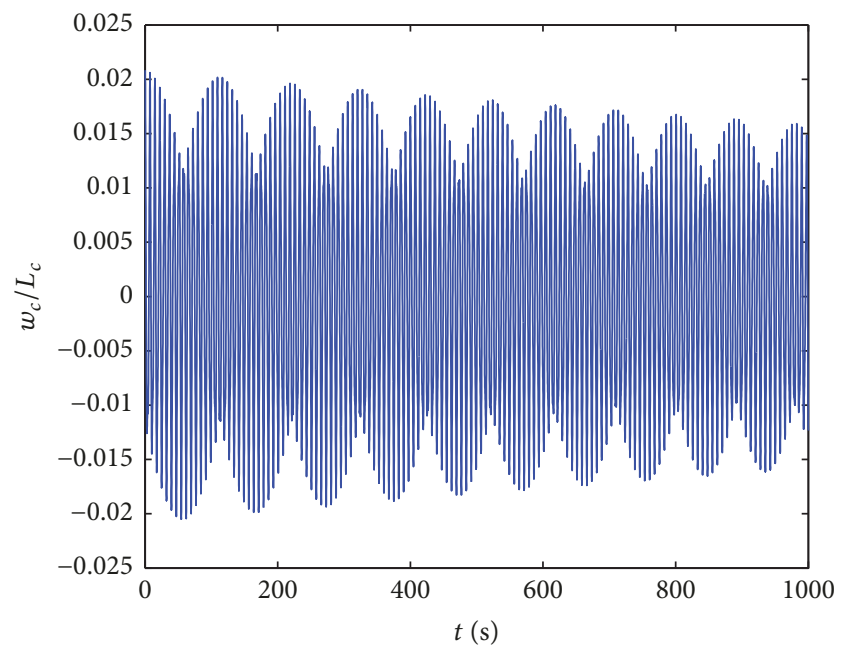

(b) Out-of-plane mid-span displacement

FIGURE 8: In-plane and out-of-plane mid-span displacement time histories of the transmission line when its initial potential energy is relatively high.

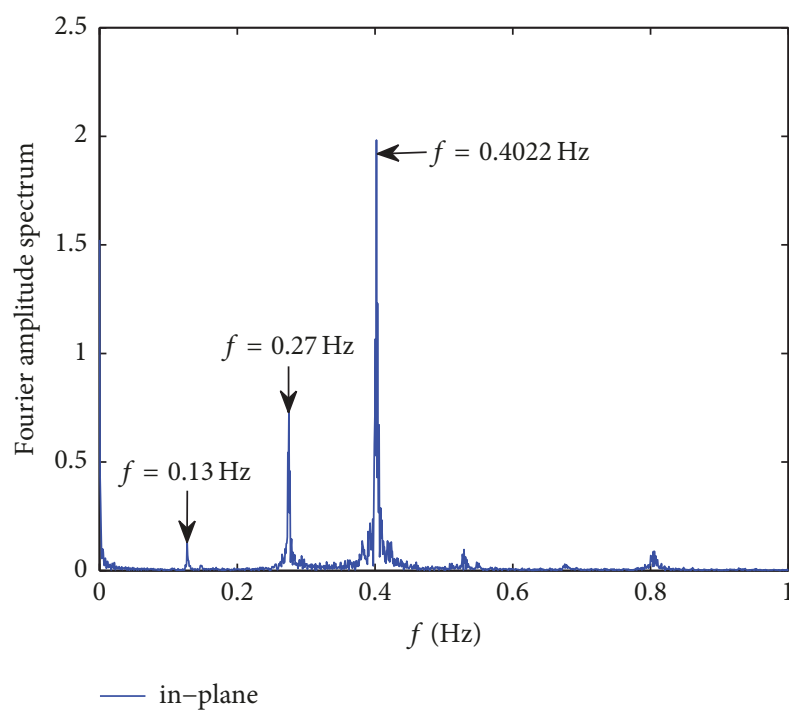

(a) In-plane

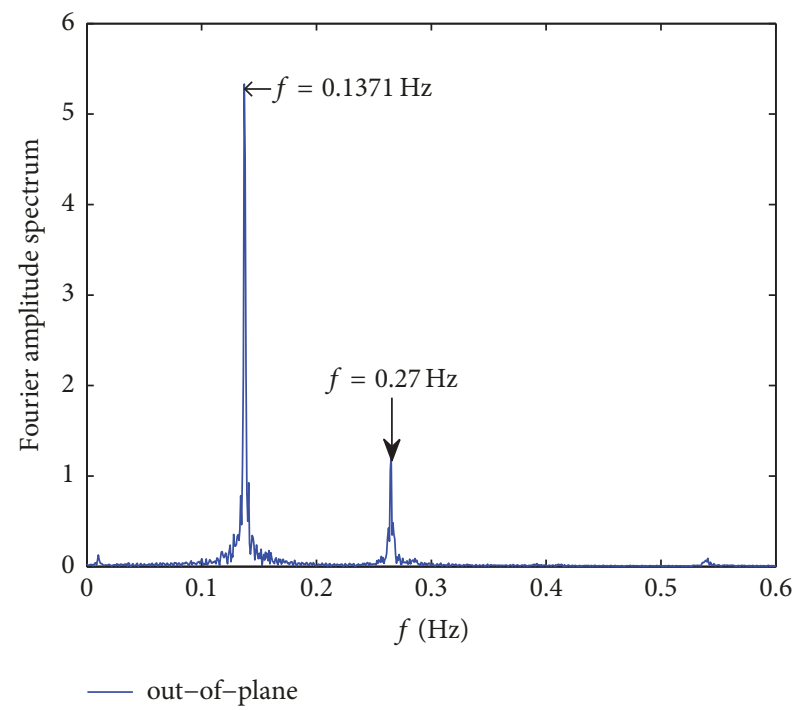

(b) Out-of-plane

FIGURE 9: Fourier spectra of the in-plane and out-of-plane mid-span displacements of the transmission line when its initial potential energy is relatively high.

line is approximately the same as the initial tension. This suggests that when the transmission line vibrates freely with relatively low initial potential energy, the effects of internal resonance on the tension in the transmission line are negligible. The spectral distribution of the dynamic tension shown in Figure 11(c) also demonstrates that when the initial potential energy of the transmission line is relatively low, the tension in the transmission line is almost unaffected by its vibration. When the initial potential energy of the transmission line is relatively high, the dynamic tension in the transmission line may reach twice the initial tension. The spectral distribution of the dynamic tension shown in
Figure 11(d) also demonstrates that the increase in the tension in the line is related to its in-plane vibration. This indicates that when the transmission line is in motion with a large amplitude, its in-plane vibration is coupled to its out-of-plane vibration; that is, when the initial out-of-plane energy of the transmission line is relatively high, significant internal resonance occurs in the transmission line when it starts vibrating, resulting in the transfer of out-of-plane vibration energy to the in-plane direction. This in turn not only gives rise to in-plane antisymmetric vibration but also causes inplane symmetric vibration, ultimately leading to a significant increase in the tension in the transmission line. 


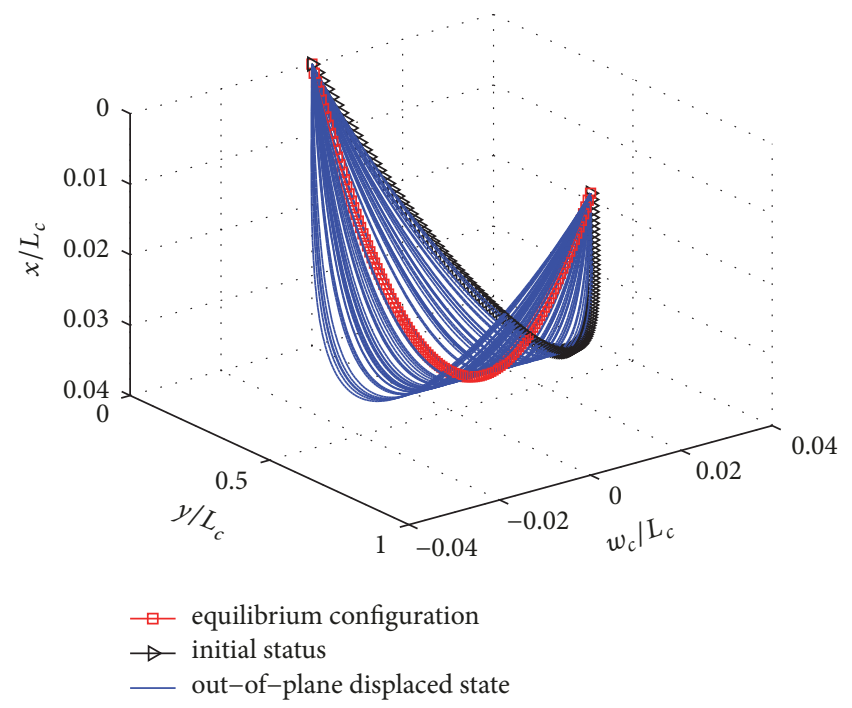

(a) Configuration of out-of-plane motion at various times

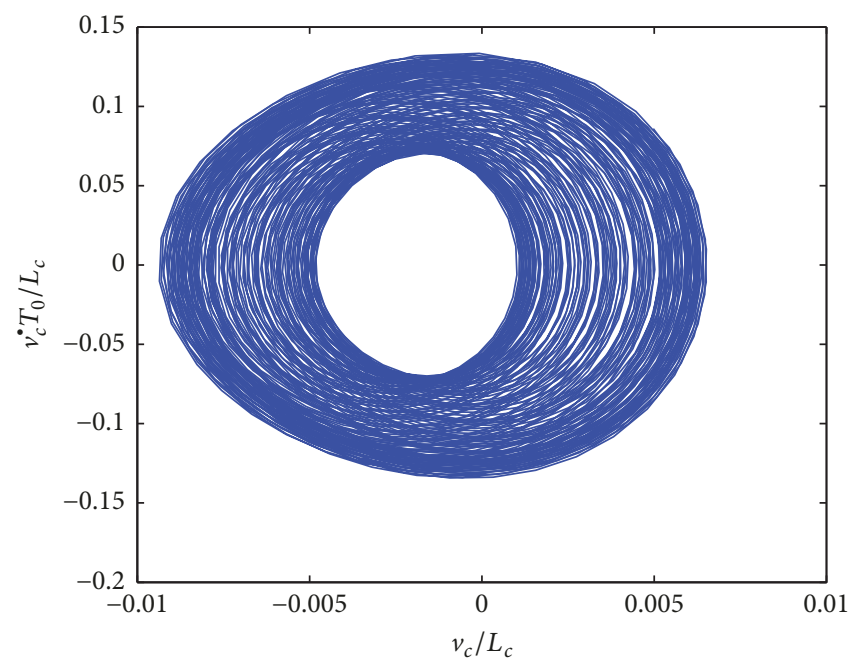

(c) In-plane mid-span phase trajectory

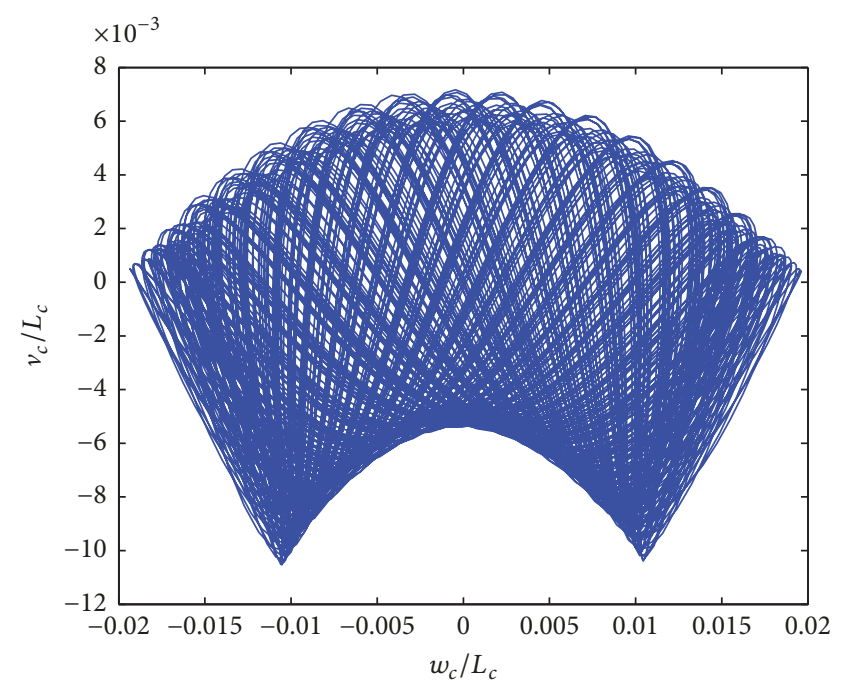

(b) In-plane and out-of-plane displacement response in the phase space

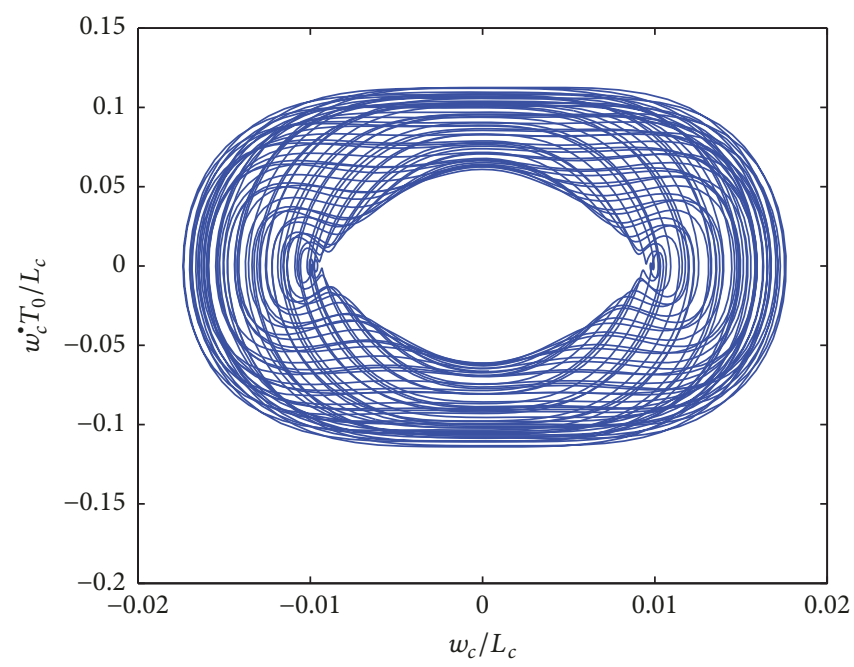

(d) Out-of-plane mid-span phase trajectory

FIGURE 10: Configuration of the out-of-plane motion and the phase-space response of the transmission line when its initial potential energy is relatively high.

4.3.3. Effects of Damping on the Nonlinear Internal Resonance of the Transmission Line. It was noted in the previous sections that the structural damping of a transmission line is very small. To effectively reduce the vibration responses of transmission lines, control devices (e.g., dampers, spiral dampers, damping spacers, and damper lines) are often installed on transmission lines in engineering practice; these control devices can generate relatively large damping. Thus, in this section, the effects of damping on the internal resonance of the transmission line are analyzed.

Here, the same initial potential energy conditions as those used in Section 4.3.2 are used, but the initial damping is varied. Figures 12 and 13 show the free vibration response of the transmission line obtained in the simulation under various damping conditions. A comparison of Figures 12 and 13 with Figures $8-10$ shows that, under the same conditions, increasing the damping of the transmission line results in a significant decrease in the displacement of and the tension in the transmission line as well as a decrease in the duration of vibration of the transmission line. This indicates that increasing the damping of the transmission line can effectively consume its vibration energy, control its vibration response amplitude, and prevent an increase in its dynamic tension but cannot hamper the occurrence of its nonlinear internal resonance.

4.4. Numerical Analysis of the Nonlinear Internal Resonance of the Transmission Line When in Forced Vibration. Based on the aforementioned analysis, we know that the natural frequencies of the lower-order modes of vibration of the overhead transmission line are relatively low. However, in an actual environment, excitation energy (primarily wind load energy) is mainly distributed in the $0-1 \mathrm{~Hz}$ band, particularly in the $0-0.2 \mathrm{~Hz}$ band. Therefore, under an external excitation 


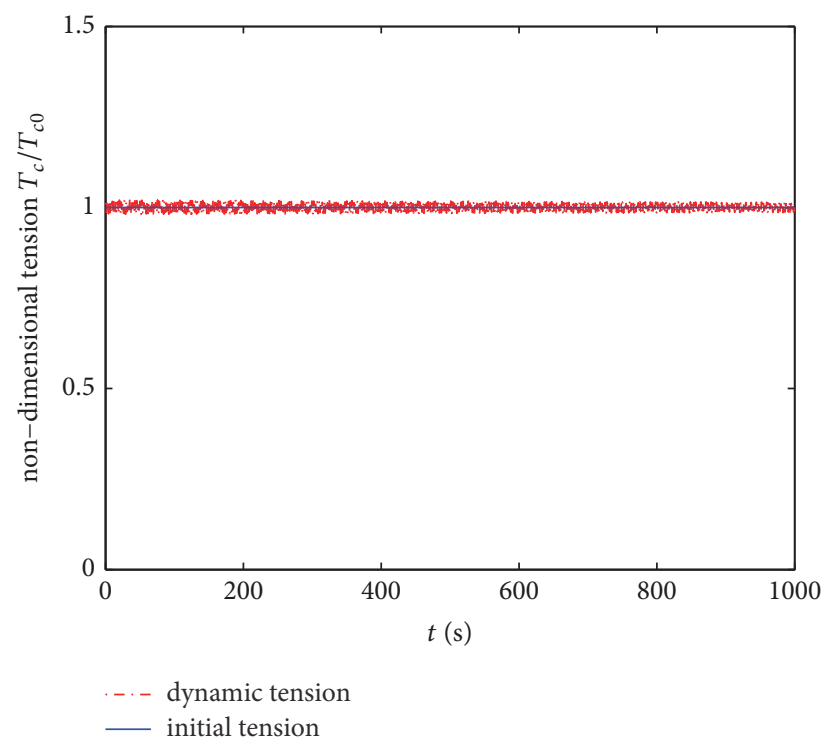

(a) Dynamic tension time history of the transmission line when its initial energy is relatively low

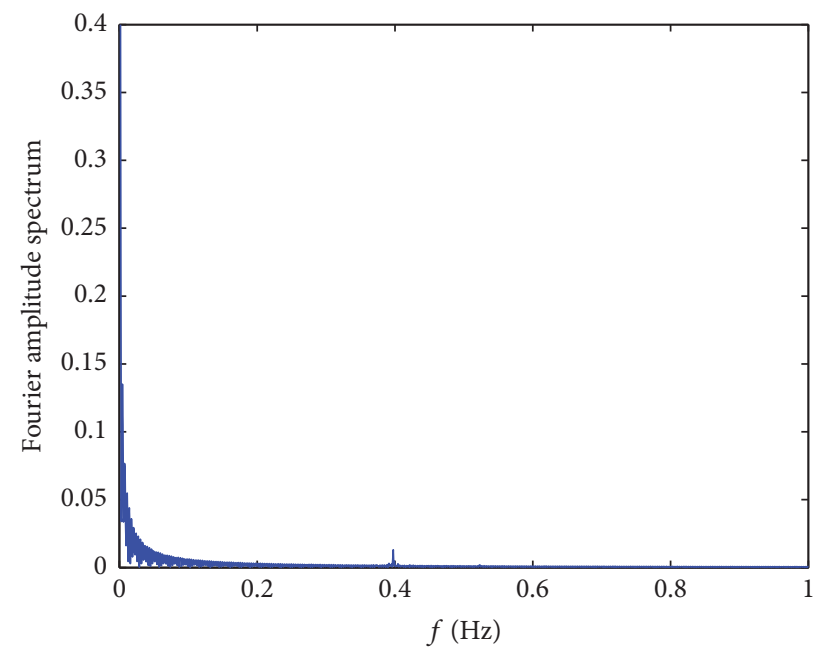

(c) Fourier spectrum of the dynamic tension in the transmission line when its initial energy is relatively low

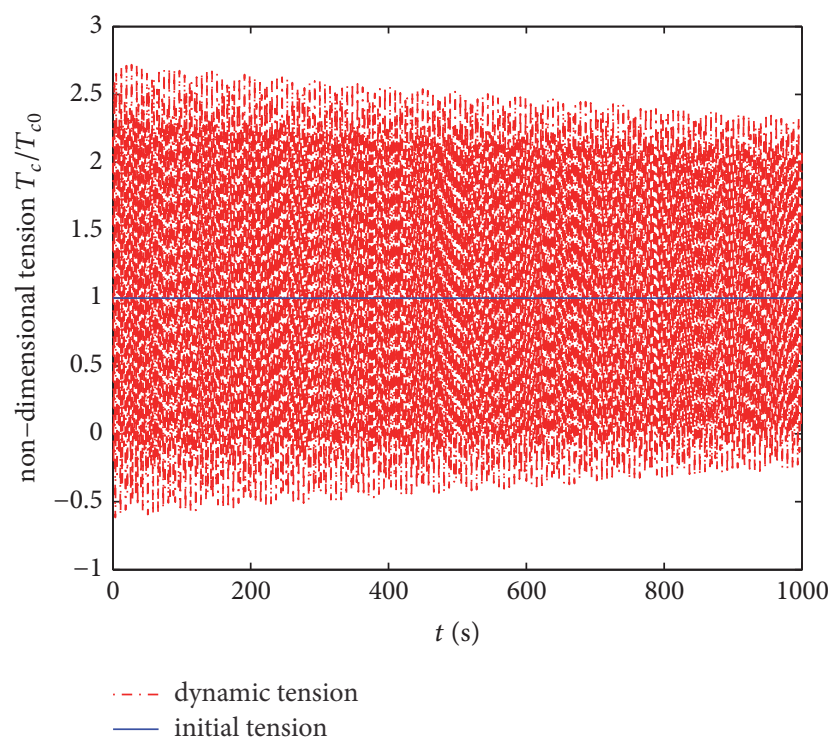

(b) Dynamic tension time history of the transmission line when its initial energy is relatively high

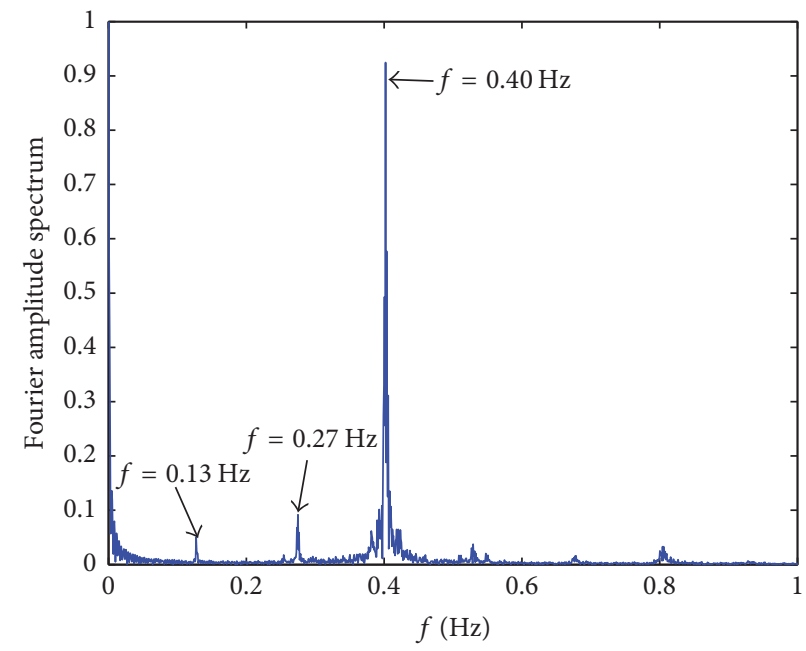

(d) Fourier spectrum of the dynamic tension in the transmission line when its initial energy is relatively high

FIgURE 11: Dynamic tension in the transmission line and its Fourier spectra under two initial energy conditions.

(e.g., a wind load), there is a relatively high probability that the transmission line will resonate with it. To further study the effects of the internal resonance of the transmission line on its wind-induced vibration, in this section the effects of the internal resonance of the transmission line under a harmonic excitation on the primary resonance are first analyzed; based on the results, the forced vibration characteristics of the transmission line under a wind load are investigated.

\subsubsection{Analysis of the Internal Resonance of the Transmission} Line under a Harmonic Excitation. Here, it is assumed that the transmission line is only subjected to an out-of-plane harmonic excitation $P_{w}=P_{0} \cos \Omega_{z} t$. The relationship between the circular frequency $\Omega_{z}$ of the excitation and the natural circular frequency $\omega_{w}$ of the out-of-plane vibration of the transmission line is characterized by introducing a detuning parameter $\sigma_{1}$, a nonnegative real number $k$, and a small parameter $\varepsilon$ :

$$
\Omega_{z}=k \omega_{w}+\varepsilon \sigma_{1} .
$$

To study the primary resonance response of the system, $k, \varepsilon$, and $\sigma_{1}$ are set to $1,0.001$, and 10 , respectively. By substituting these values into (51), the frequency $f_{z}$ of the outof-plane excitation is determined: $f_{z}=\Omega_{z} /(2 \pi)=0.146 \mathrm{~Hz}$. The natural frequency $f_{w}$ of the first-order out-of-plane vibration of the transmission line that is directly induced by the excitation is as follows: $f_{w}=\omega_{w} /(2 \pi)=0.136 \mathrm{~Hz}$. $f_{z}$ approximately equals $f_{w}$. Thus, it can be considered that $\Omega_{z} \approx \omega_{w}$. 


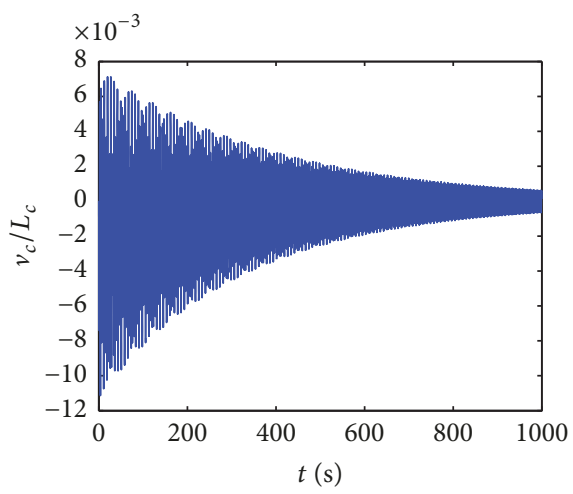

(a) In-plane mid-span displacement time history

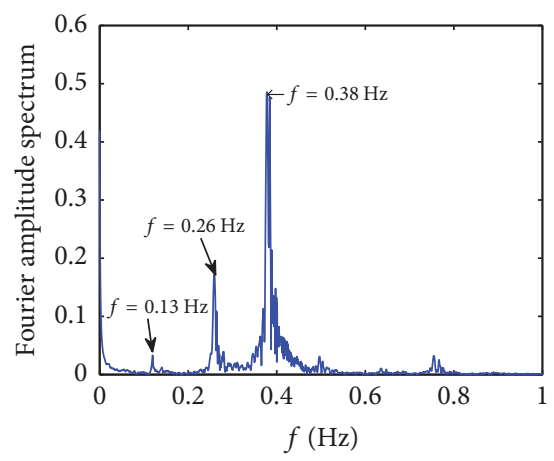

— in-plane

(c) Fourier spectrum of the in-plane mid-span displacement

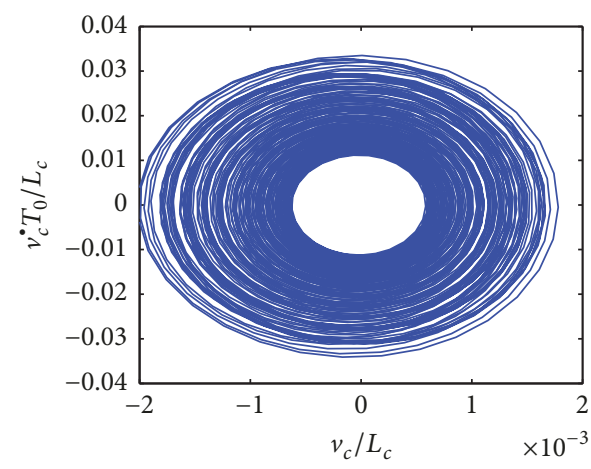

(e) In-plane mid-span phase trajectory

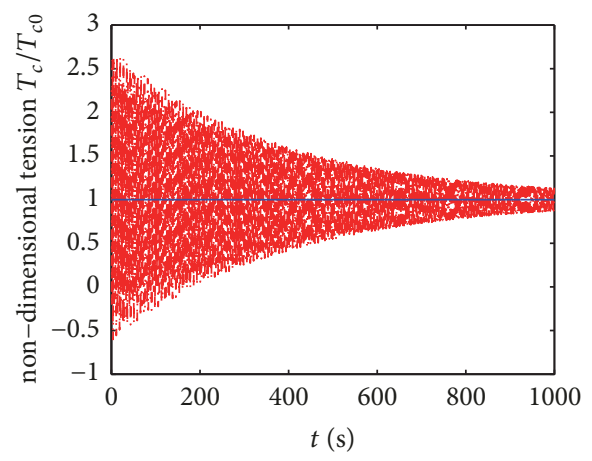

-.- dynamic tension

— initial tension

(g) Dynamic tension time history

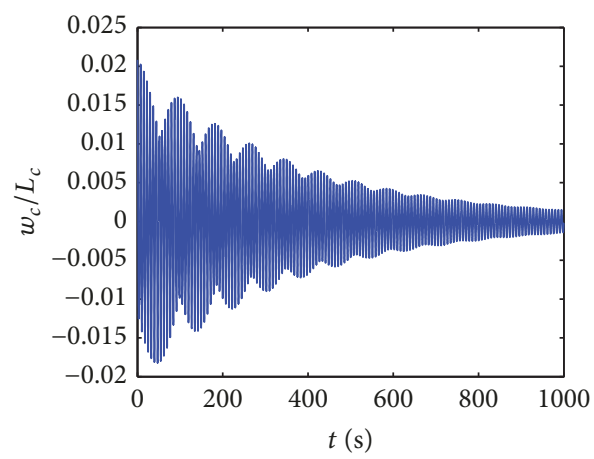

(b) Out-of-plane mid-span displacement time history

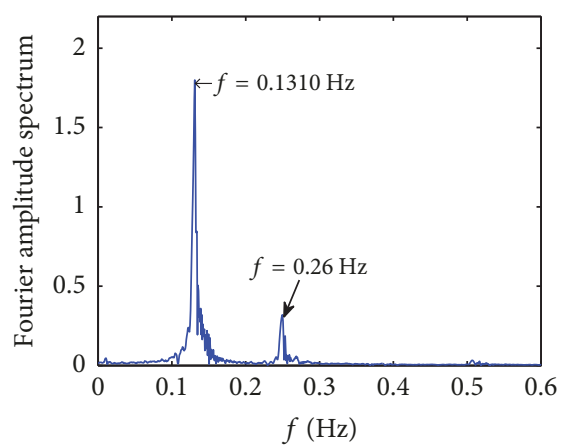

_ out-of-plane

(d) Fourier spectrum of the out-of-plane midspan displacement

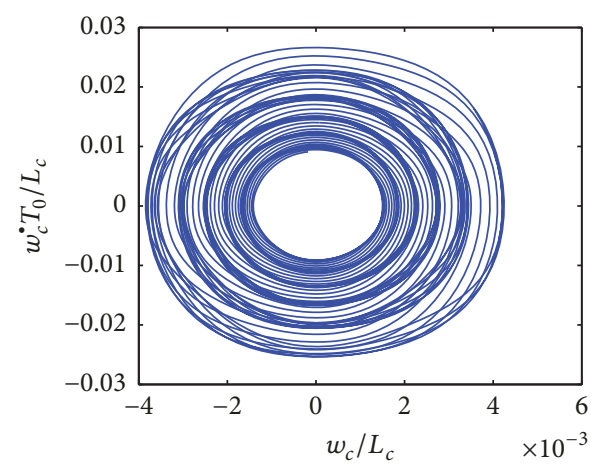

(f) Out-of-plane mid-span phase trajectory

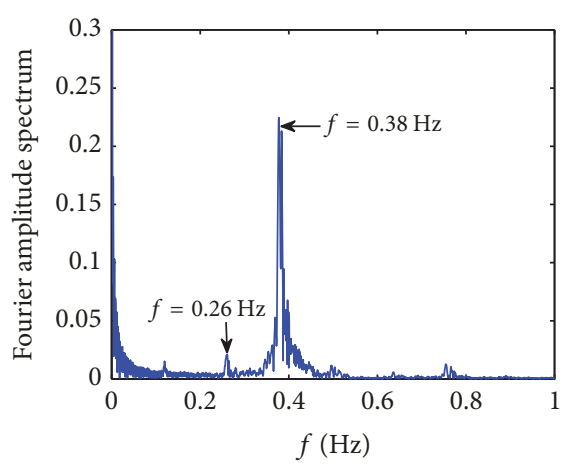

(h) Fourier spectrum of the dynamic tension

FIGURE 12: Free vibration response of the transmission line when its damping $c_{c}$ is set to 0.01 . 


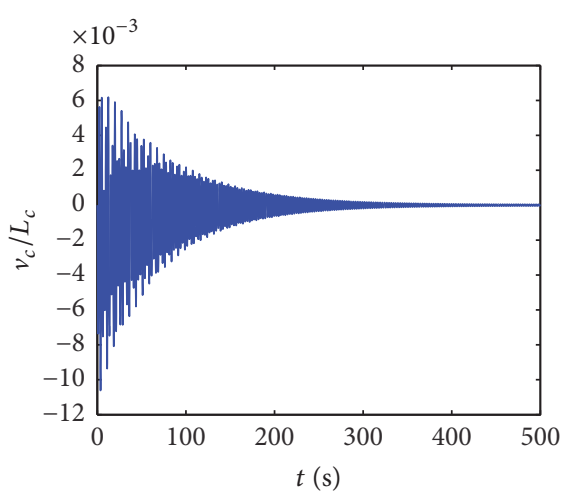

(a) In-plane mid-span displacement

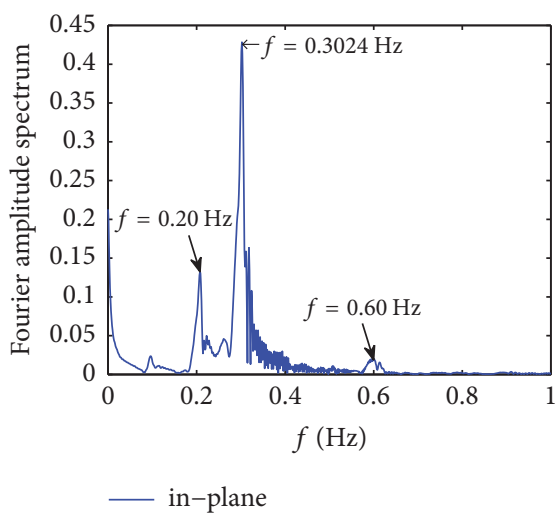

(c) Fourier spectrum of the in-plane mid-span displacement

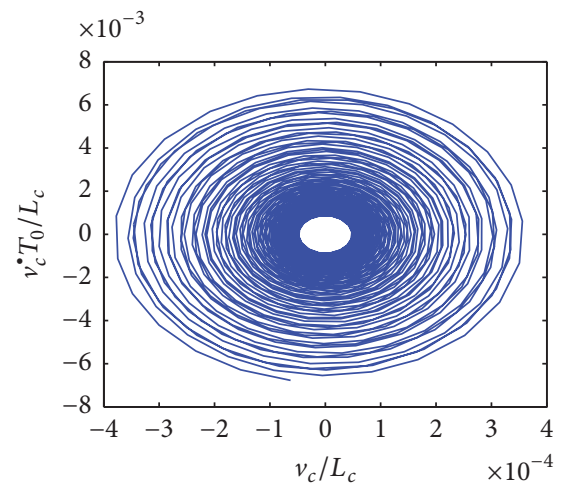

(e) In-plane mid-span phase trajectory

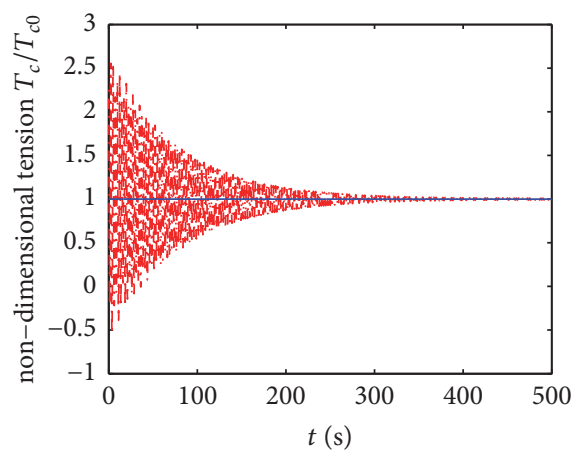

-.- dynamic tension

— initial tension

(g) Dynamic tension time history

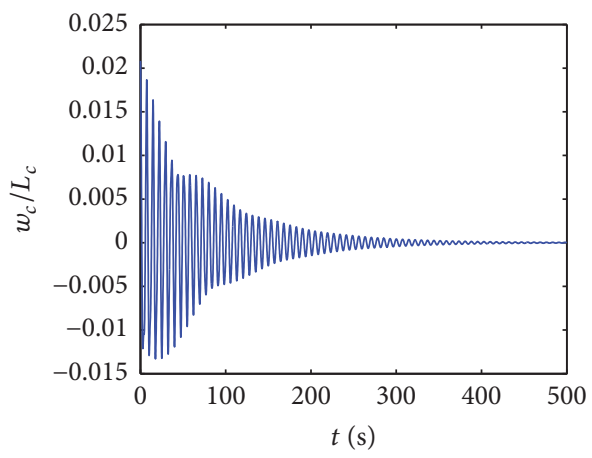

(b) Out-of-plane mid-span displacement

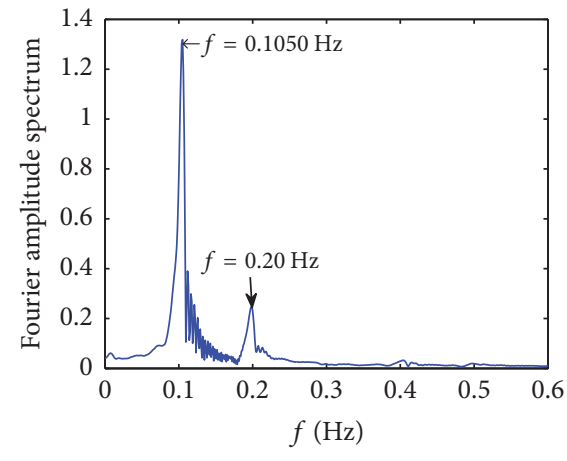

_ out-of-plane

(d) Fourier spectrum of the out-of-plane midspan displacement

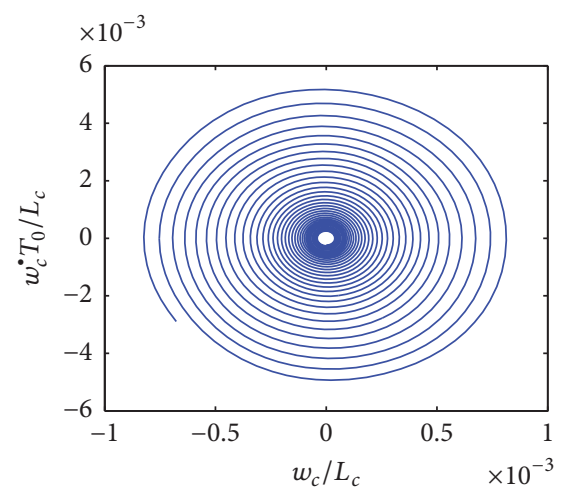

(f) Out-of-plane mid-span phase trajectory

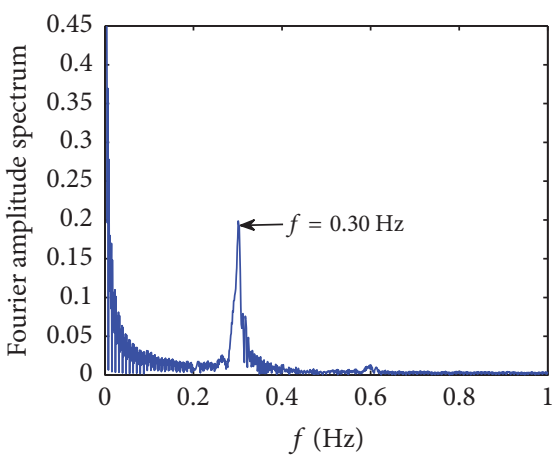

(h) Fourier spectrum of the dynamic tension

FIGURE 13: Free vibration response of the transmission line when its damping $c_{c}$ is set to 0.05 . 
Let us set the amplitude $P_{0}$ of the generalized excitation load to $3305 \mathrm{~N}$ and the initial conditions for simulation to $q_{v}=0, \dot{q}_{v}=0, q_{w}=0.1, \dot{q}_{w}=0.00005$. By substituting these values into (54), the nonlinear forced vibration response of the transmission line under various damping conditions is determined. Figures 14-17 show the numerical simulation results obtained when the damping $c_{c}$ is set to $0.001,0.01,0.05$, and 0.1 , respectively.

As demonstrated in Figure 14, the out-of-plane vibration of the transmission line directly induced by the excitation has a very large amplitude, suggesting that the transmission line is forced to resonate when $\Omega_{z} \approx \omega_{w}$. Due to the internal resonance, the vibration energy of the transmission line transfers from the out-of-plane direction to the in-plane direction, which is not directly under excitation. In addition, the in-plane vibration of the transmission line also exhibits resonance characteristics. In other words, due to the internal resonance, the forced resonance of the transmission line can involve several modes of resonance. When only considering the small structural damping, the resonance amplitude of the transmission line will jump; that is, within a certain period of time, the response occurs at a relatively small amplitude, and vibration with a relatively large amplitude occurs at a certain time and can continue steadily for an extended period of time. This differs from the resonance of a linear system. Due to the nonlinear coupling and internal resonance, the forced resonance modulates the vibration frequency of the system, and the frequencies of the out-of-plane and inplane vibration of the transmission line are no longer linear natural frequencies and are also not the same as the excitation frequency but instead are manifested as a relatively wide distribution of vibration energy (Figures 14(c) and 14(d)). As demonstrated in the phase diagrams of the in-plane and outof-plane response (Figures 14(e) and 14(f)), the in-plane and out-of-plane response is aperiodic with an amplitude that jumps. Considering that the external excitation has a very large amplitude due to the nonlinear internal resonance, the forced resonance excites the transmission line to resonate internally, resulting in a sharp increase in the dynamic tension in the transmission line. In addition, because of the inplane vibration amplitude jumps, a corresponding jumping phenomenon is also observed in the dynamic tension time history curve (Figure $14(\mathrm{~g})$ ). As shown in the Fourier spectrum of the dynamic tension (Figure 14(h)), the dynamic tension in the transmission line is mainly significantly affected by the in-plane vibration. Because its amplitude is far greater than the design-breaking load, the dynamic tension may cause the transmission to break.

Figure 15 shows the simulation results obtained by increasing the damping $c_{c}$ to 0.01 , while retaining the other conditions. As demonstrated in Figure 15, amplitude jumps are eliminated due to the damping effects, but internal resonance still exists. The in-plane vibration of the transmission line not directly induced by the excitation has a large amplitude, which are of the same order of magnitude of the out-ofplane vibration. In addition, the frequency modulation by the forced resonance becomes more complicated compared to the situation in which the damping is small (Figures 15(c) and $15(\mathrm{~d})$ ). As demonstrated in the phase trajectory of the steady-state response (Figures 15(e) and 15(f)), there are still jumps in the in-plane vibration amplitude of the transmission line and the corresponding dynamic tension amplitude, and the maximum value of these jumps can be as high as 3 times the initial tension. This indicates that it is still possible for the transmission line to be broken under these conditions.

Figures 16 and 17 show the simulation results that are obtained when the damping $c_{c}$ is further increased to 0.05 and 0.1 , respectively, while retaining the other conditions. As demonstrated in Figures 16 and 17, due to the large damping effects, the vibration amplitude of the transmission line reaches a stable state within a short period of time; however, the large damping is unable to prevent the occurrence of internal resonance, and the in-plane vibration still has a very large amplitude, the numerical value of which is commensurate with that of the out-of-plane vibration. The dynamic tension in the transmission line when it is in steady-state vibration can still reach approximately three times the initial tension. This suggests that even under relatively large damping effects, the forced resonance of the transmission line cannot be maintained as the steady-state mode of motion directly induced by the excitation but is instead manifested as the nonlinear vibration of coupled modes. Due to the frequency-modulating effect of the forced resonance, the natural frequencies of the modes of vibration of the transmission line directly induced by the excitation more accurately approach the excitation frequency, and, correspondingly, the modulated frequencies inherit the relationships between the original linear natural frequencies: they are either closely spaced with or multiples of one another. In addition, the vibration still contains higher-order modes of vibration.

As demonstrated in the phase trajectories of the response (Figures 16(e), 16(f), 17(e), and 17(f)), when its damping is relatively high, the resonance response of the transmission line reaches saturation. In other words, because the nonlinear vibration equations for the transmission line exhibit quadratic nonlinearity and the linear natural vibration frequencies of the transmission line satisfy the $\omega_{w} \approx \omega_{v}$ and $\omega_{v} \approx 2 \omega_{w}$ conditions, when the frequency $\Omega_{z}$ of the external excitation is approximately equal to $\omega_{w}$ and the excitation amplitude meets a certain condition, the response energy of the mode of vibration directly induced by the excitation reaches saturation, and all the input excitation energy enters another mode. Thus, while its damping can be increased by installing energy dissipating equipment in engineering practice, the motion of a transmission line exhibits coupled in-plane and out-of-plane vibration characteristics due to the nonlinear internal resonance; in addition, a transmission line can still continue to vibrate with a relatively large amplitude under an external excitation with a relatively large amplitude, and the vibration energy continuously transfers between relevant modes without being attenuated. Due to the presence of this response saturation phenomenon, the dynamic tension in a transmission line when in vibration can still possibly reach a higher level even when its damping is set to a relatively large value, which is disadvantageous to practical engineering.

To compare the vibration response of the transmission line when not in resonance with the vibration response of 


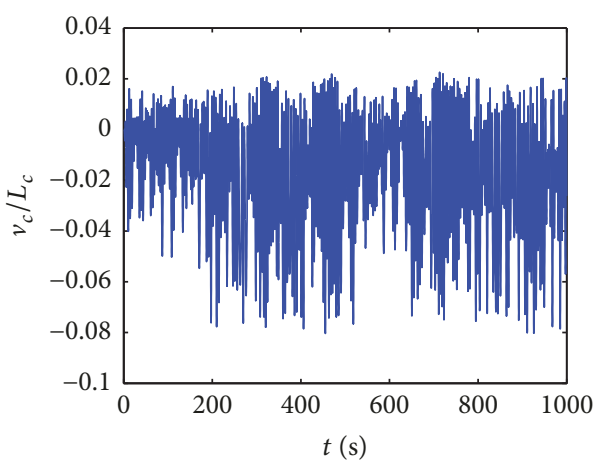

(a) In-plane mid-span displacement time history

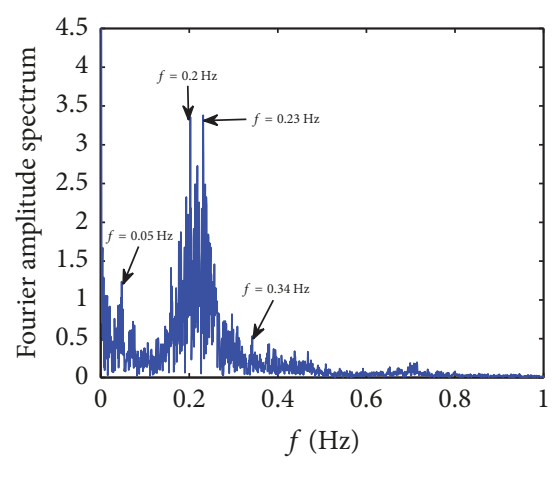

_ in-plane

(c) Fourier spectrum of the in-plane mid-span displacement

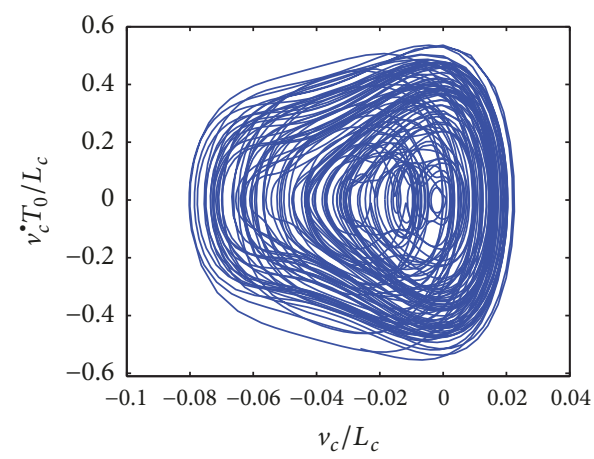

(e) In-plane mid-span phase trajectory

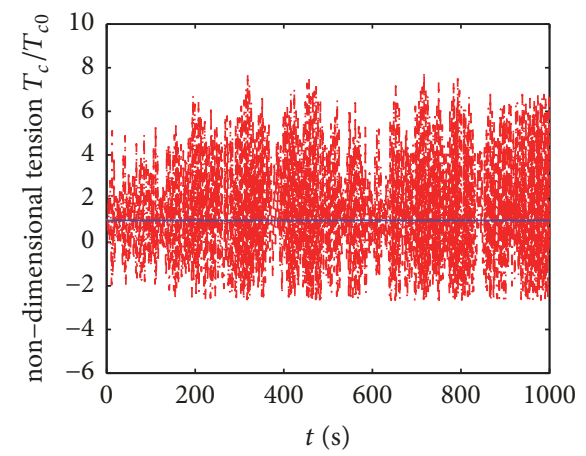

-. - dynamic tension

_ initial tension

(g) Dynamic tension time history

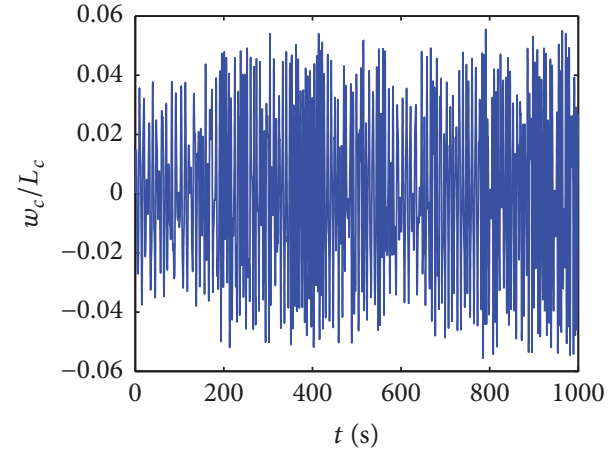

(b) Out-of-plane mid-span displacement time history

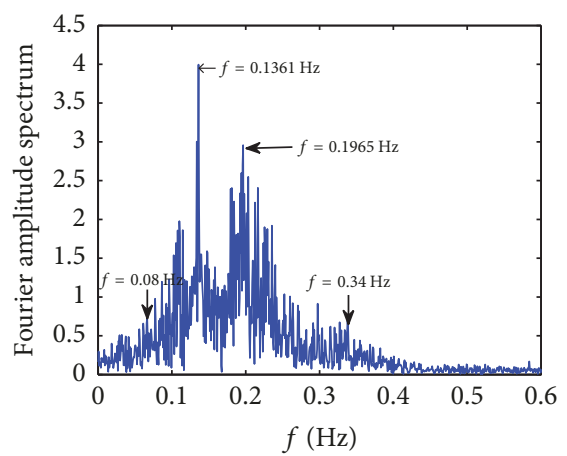

_ out-of-plane

(d) Fourier spectrum of the out-of-plane midspan displacement

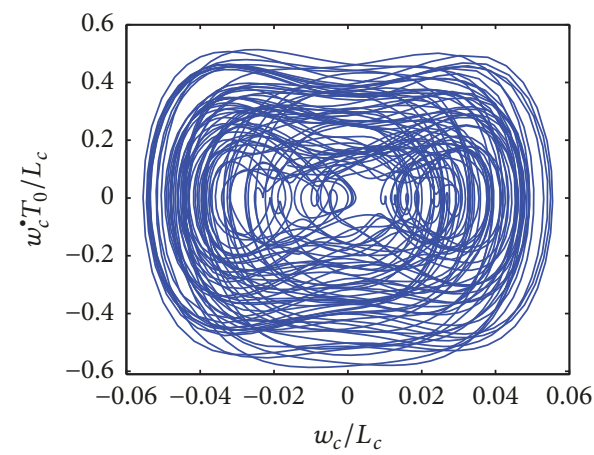

(f) Out-of-plane mid-span phase trajectory

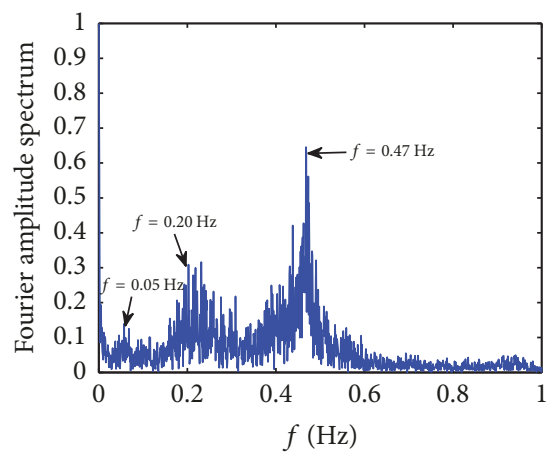

(h) Fourier spectrum of the dynamic tension

Figure 14: Primary resonance response of the transmission line when its damping $c_{c}$ is set to 0.001 . 


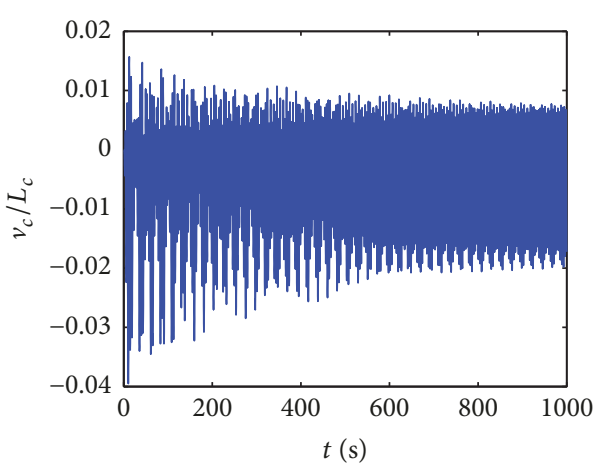

(a) In-plane mid-span displacement

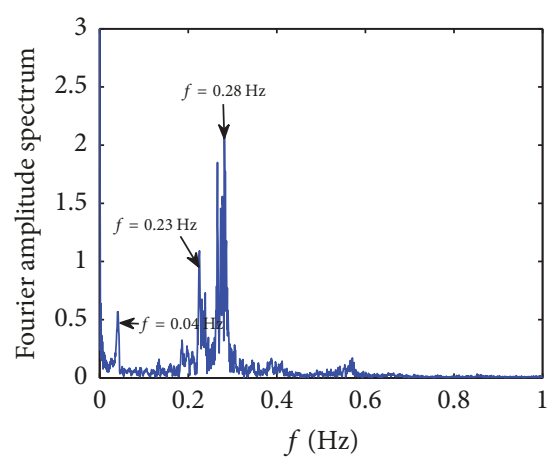

_ in-plane

(c) Fourier spectrum of the in-plane mid-span displacement

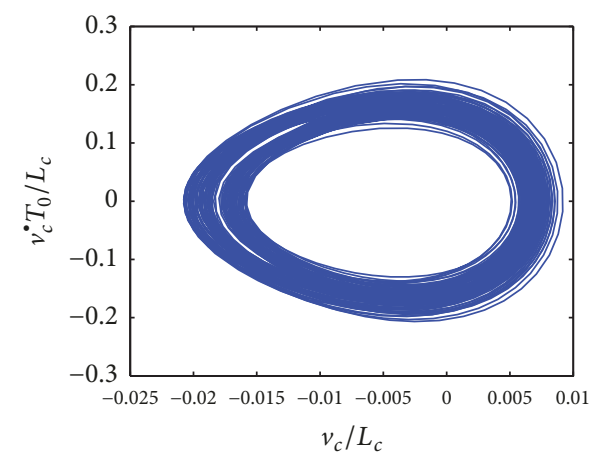

(e) In-plane mid-span phase trajectory

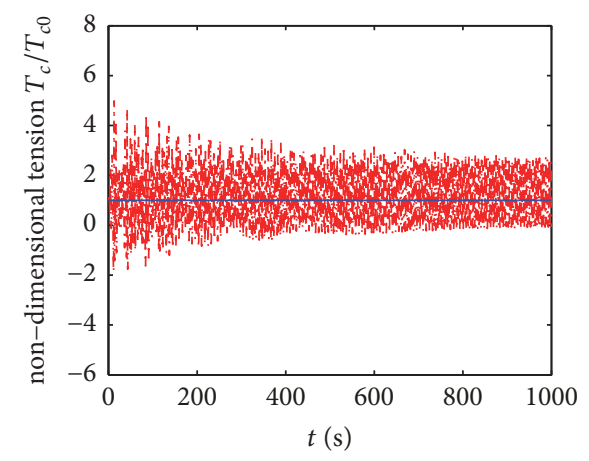

... dynamic tension

(g) Dynamic tension time history

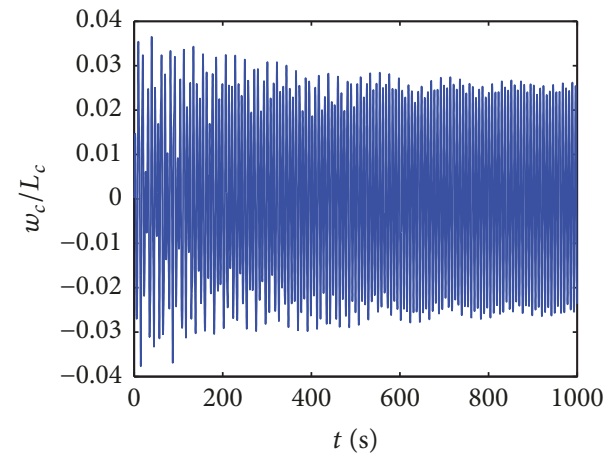

(b) Out-of-plane mid-span displacement

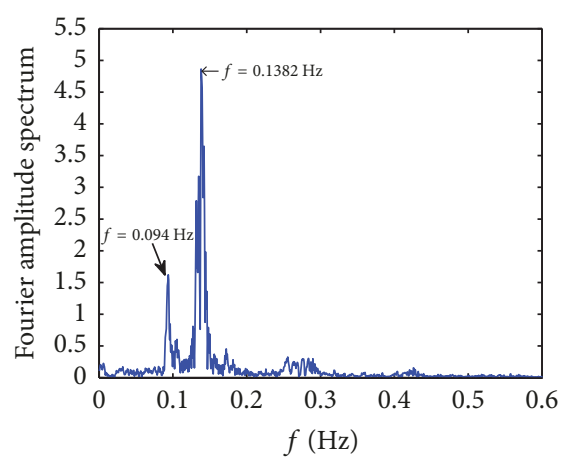

_ out-of-plane

(d) Fourier spectrum of the out-of-plane midspan displacement

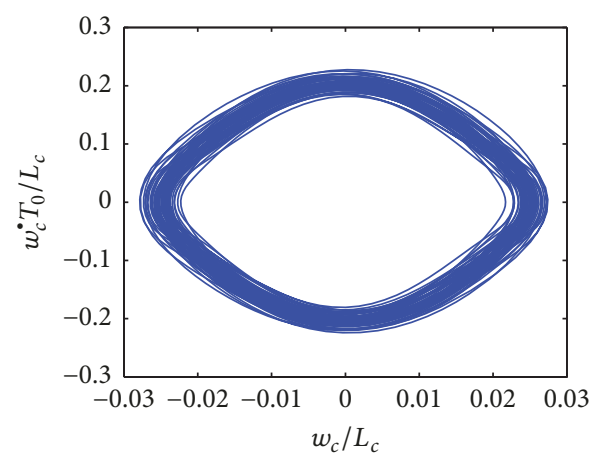

(f) Out-of-plane mid-span phase trajectory

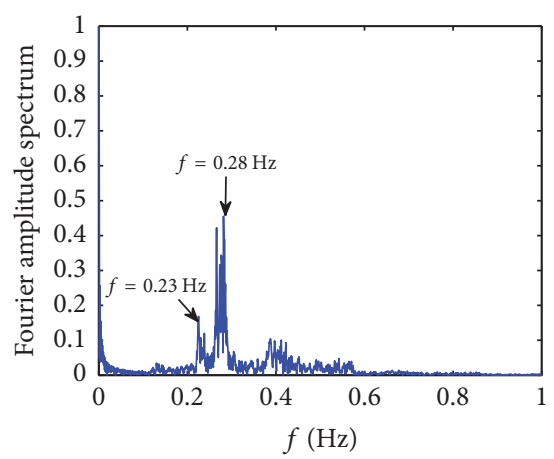

(h) Fourier spectrum of the dynamic tension

FIGURE 15: Primary resonance response of the transmission line when its damping $c_{c}$ is set to 0.01 . 


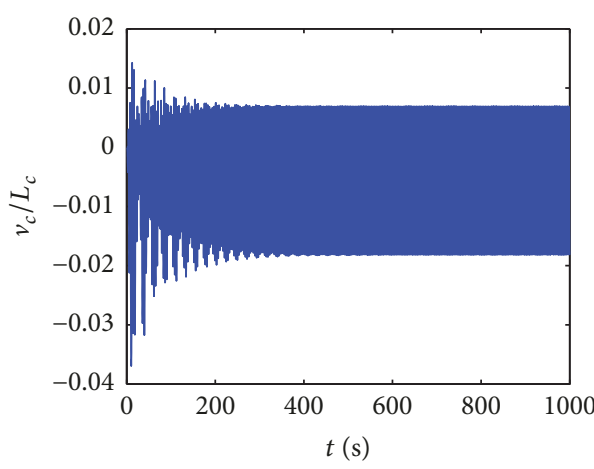

(a) In-plane mid-span displacement

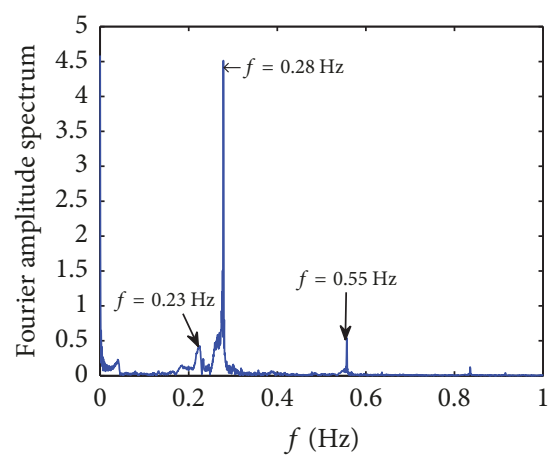

— in-plane

(c) Fourier spectrum of the in-plane mid-span displacement

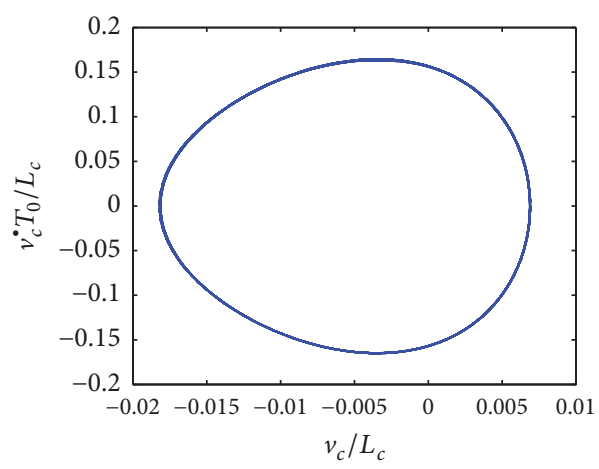

(e) In-plane mid-span phase trajectory

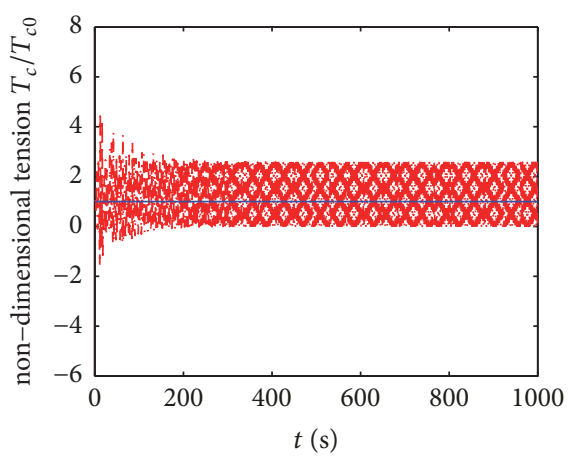

-.- dynamic tension

- initial tension

(g) Dynamic tension time history

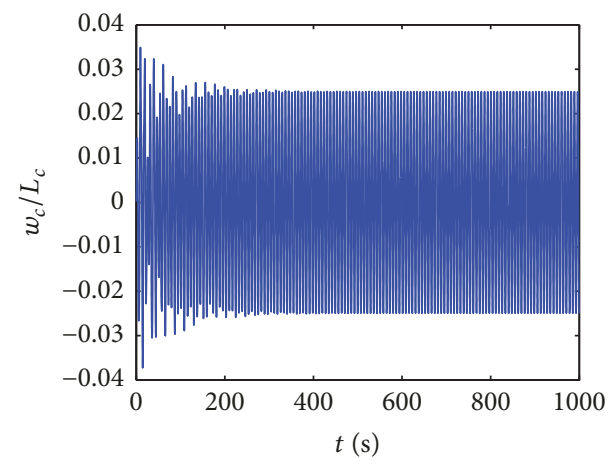

(b) Out-of-plane mid-span displacement

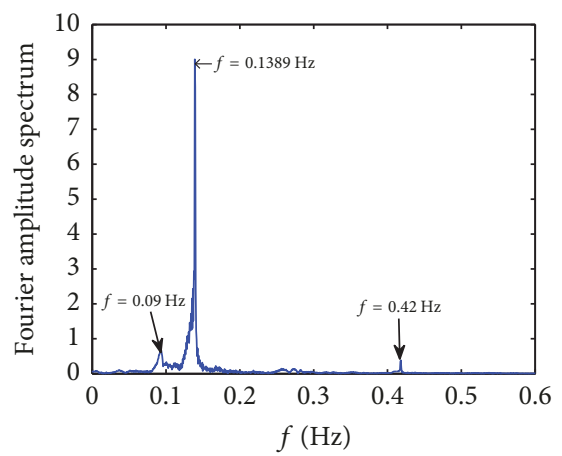

_ out-of-plane

(d) Fourier spectrum of the out-of-plane midspan displacement

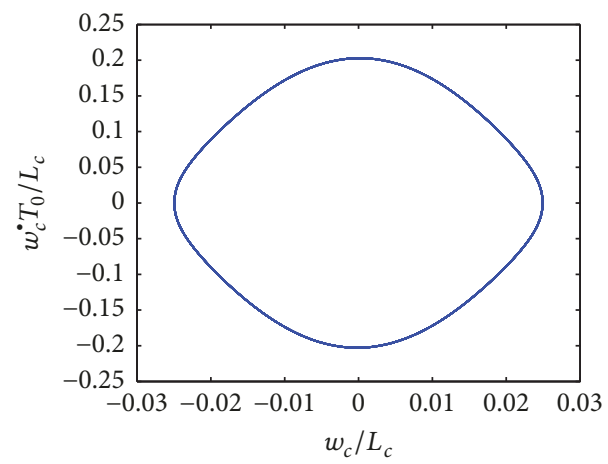

(f) Out-of-plane mid-span phase trajectory

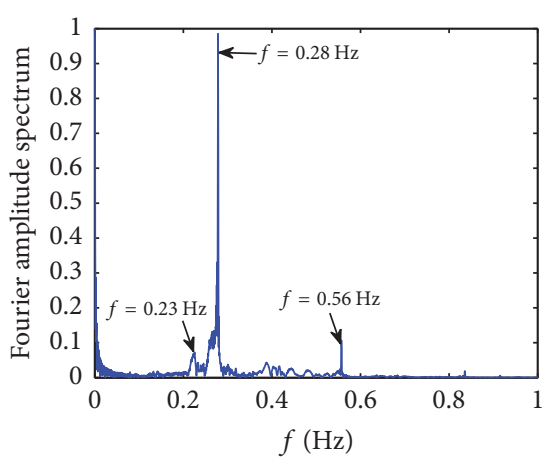

(h) Fourier spectrum of the dynamic tension

FIGURE 16: Primary resonance response of the transmission line when its damping $c_{c}$ is set to 0.05 . 


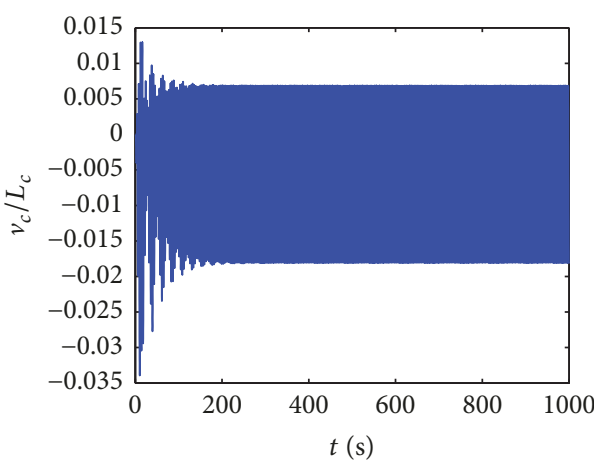

(a) In-plane mid-span displacement

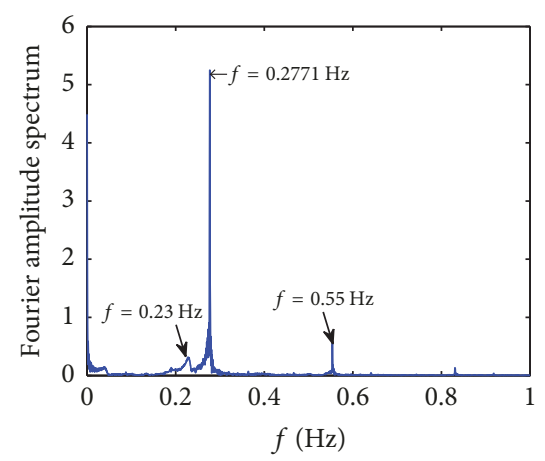

_ in-plane

(c) Fourier spectrum of the in-plane mid-span displacement

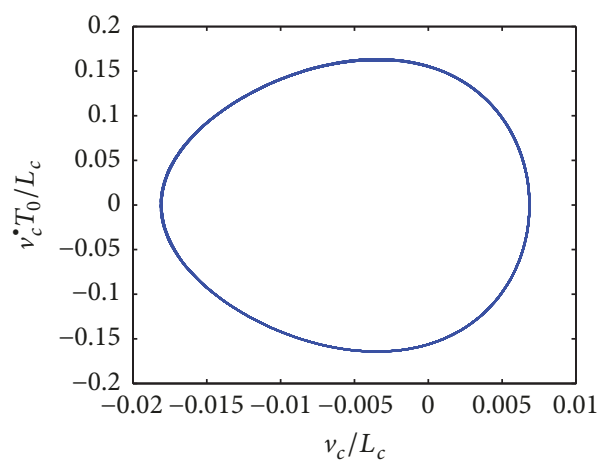

(e) In-plane mid-span phase trajectory

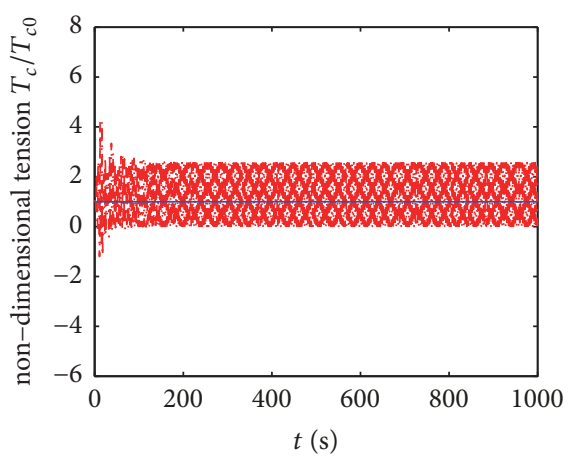

-.- dynamic tension

- initial tension

(g) Dynamic tension time history

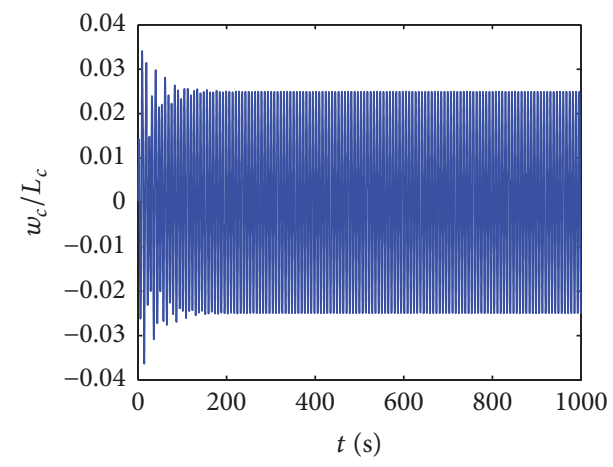

(b) Out-of-plane mid-span displacement

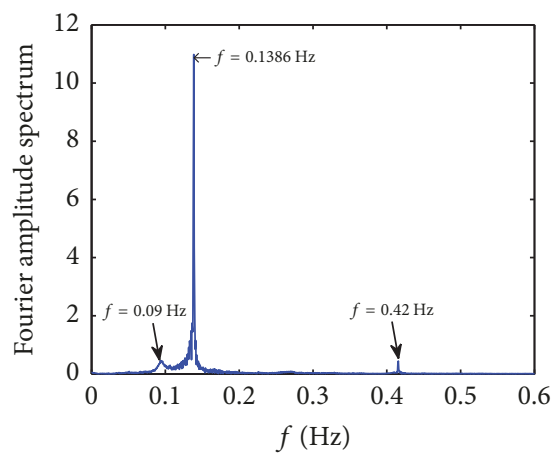

_ out-of-plane

(d) Fourier spectrum of the out-of-plane midspan displacement

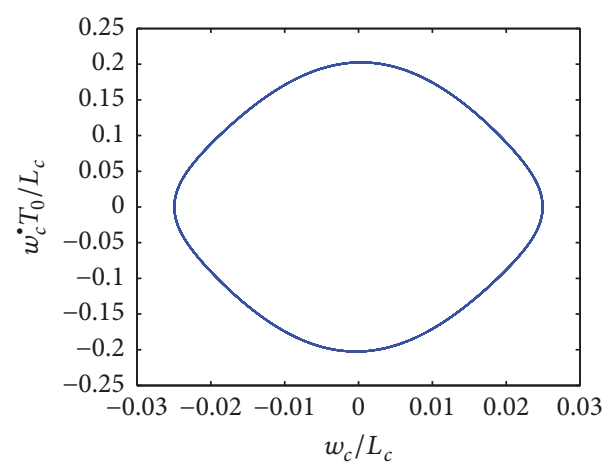

(f) Out-of-plane mid-span phase trajectory

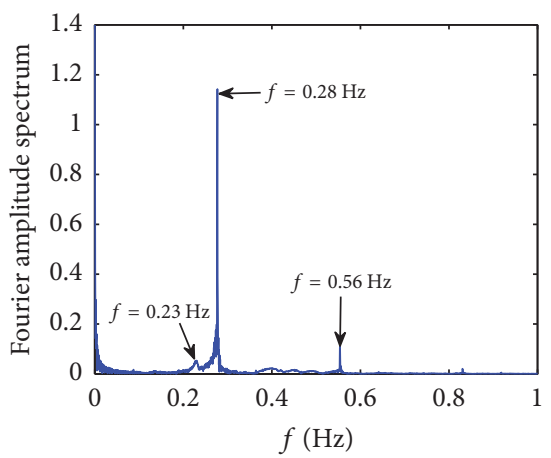

(h) Fourier spectrum of the dynamic tension

FIgURE 17: Primary resonance response of the transmission line when its damping $c_{c}$ is set to 0.1 . 
the transmission line when in resonance, the following set of parameters is selected: frequency $f_{z}$ of the external excitation: $0.68 \mathrm{~Hz}$; damping $c_{c}$ : 0.001 ; all the other parameters are the same as those used to simulate the transmission line when in resonance. Figure 18 shows the simulation results. A comparison of Figures 14 and 18 shows that when the external excitation amplitude and the damping of the transmission line remain unchanged, the vibration amplitude of the transmission line is far smaller when not in resonance than when in resonance. While an internal resonance phenomenon can still be observed numerically when the transmission line is not in resonance, the internal resonanceinduced in-plane vibration amplitude is very small and is negligible. When the transmission line is not in resonance, the vibration of the system is primarily composed of the out-of-plane forced vibration induced by the out-of-plane excitation (Figure 18(d)); in addition, the dynamic tension in the transmission line changes to a relatively small extent (Figure $18(\mathrm{~g})$ ). It can be seen that internal resonance has a great influence on the main resonance of transmission line, so the design of transmission line needs to consider its influence reasonably.

\subsubsection{Analysis of the Internal Resonance of the Transmission} Line When in Wind-Induced Vibration. Under actual operating conditions, overhead transmission lines will inevitably undergo wind-induced vibration. Therefore, in this section, the nonlinear vibration characteristics of the transmission line under a wind load are studied. Considering that the vertical in-plane wind load on a transmission line is generally very small, to simplify the analytical process, it is assumed that the wind load acts in the out-of-plane direction of the transmission line. The quasi-steady hypothesis is used in calculation: when calculating the wind load on the transmission line, only the effects of the vibration of the transmission line on the wind speed are considered, whereas the aerodynamic coupling effects are not considered: that is, the load terms $P(x, t)$ and $P(z, t)$ in (26) and (27) can be expressed as follows:

$$
\begin{aligned}
& P(x, t)=0.5 \rho_{a} D_{c} C_{D}\left(v_{a-x}-\dot{v}_{c}\right)^{2}, \\
& P(z, t)=0.5 \rho_{a} D_{c} C_{D}\left(v_{a-z}-\dot{w}_{c}\right)^{2},
\end{aligned}
$$

where $\rho_{a}$ represents the air density; $D_{c}$ represents the windward width of the transmission line; $C_{D}$ represents the shape factor of the transmission line; $v_{a-x}$ and $v_{a-z}$ represent the wind speeds along the $x$ - and $z$-axis directions, respectively; $\dot{v}_{c}, \dot{w}_{c}$ represent the in-plane and out-of-plane vibration velocities of the transmission line, respectively.

By substituting (60) into (26) and (27) and finding the first-order modal solutions for the in-plane and out-of-plane vibration using Galerkin's modal truncation method, the continuous partial differential equations can be transformed to discrete ordinary partial equations, as shown in (30), in which the factors $a_{7}, b_{6}, P_{v}$, and $P_{w}$ are calculated using the following equations (all the other factors are calculated using the same equations as introduced in Section 2.2):

$$
\begin{aligned}
& a_{7}=\int_{0}^{L_{c}}\left(c_{c}+\rho_{a} D_{c} C_{D} v_{a-x}\right) \varphi_{v}^{2}(y) d y, \\
& b_{6}=\int_{0}^{L_{c}}\left(c_{c}+\rho_{a} D_{c} C_{D} v_{a-z}\right) \varphi_{w}^{2}(y) d y, \\
& P_{v}=\int_{0}^{L_{c}} 0.5 \rho_{a} D_{c} C_{D}\left(v_{a-x}\right)^{2} \varphi_{v}(y) d y, \\
& P_{w}=\int_{0}^{L_{c}} 0.5 \rho_{a} D_{c} C_{D}\left(v_{a-z}\right)^{2} \varphi_{w}(y) d y .
\end{aligned}
$$

Wind speeds used in this section are selected from the database of wind speeds recorded by the field wind measurement platform installed on the transmission line (Figure 3). Three wind speed time histories with different average winds are selected, which are three wind speed records with an average value $U_{0}$ of $5.2,9.56$, and $20.1 \mathrm{~m} / \mathrm{s}$, respectively. These three wind speeds are equivalent to the Beaufort Scales 3, 5, and 8, respectively. The in-plane vertical wind load on the transmission line is converted from the vertical fluctuating wind speed recorded by the wind speed measurement device and its value is generally small and negligible. Figure 19 shows the frequency spectra corresponding to the aforementioned three wind speed time histories. As demonstrated in Figure 19, the wind speed energy is mainly distributed in the $0-1 \mathrm{~Hz}$ band.

Equation (30) is solved using a higher-order Runge-Kutta method. The damping $c_{c}$ of the transmission line is set to 0.01 , and the initial conditions for calculation are set as follows:

$$
\begin{aligned}
q_{v} & =0, \\
\dot{q}_{v} & =0, \\
q_{w} & =0.5, \\
\dot{q}_{w} & =0.00005 .
\end{aligned}
$$

Based on Tables 3 and 4, we know that the natural frequencies of the first-order in-plane and out-of-plane vibration of the transmission satisfy the following condition: $\omega_{v} \approx$ $3 \omega_{w}$. Figures 20-22 show the numerical simulation results with respect to the vibration response of the transmission line under a wind load.

As demonstrated in Figure 20, when $U_{0}=5.2 \mathrm{~m} / \mathrm{s}$, the out-of-plane mid-span vibration amplitude of the transmission line can exceed $1 \mathrm{~m}$, and its in-plane mid-span vibration amplitude is far greater than its cross-sectional diameter; in addition, the in-plane vibration is associated with a noticeable jumping phenomenon (Figure 20(a)). This indicates that the transmission line also undergoes internal resonance when in wind-induced vibration and that the vibration energy transfers between the out-of-plane and the in-plane modes of vibration. The overall vibration of the transmission line is manifested as coupled in-plane and out-of-plane vibration (Figure 20(b)). In-plane vibration amplitude jumps can also be found by observing the phase trajectories of the transmission line (Figures 20(c) and 20(d)). The out-of-plane vibration is a complex aperiodic motion. Due to the excitation from the wind load and the frequency-modulating effect of 


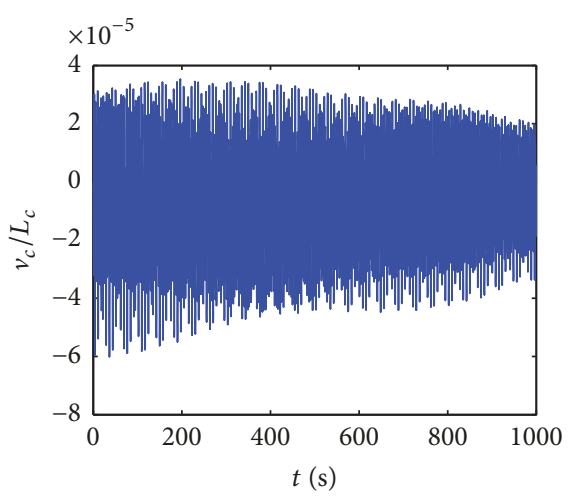

(a) In-plane mid-span displacement

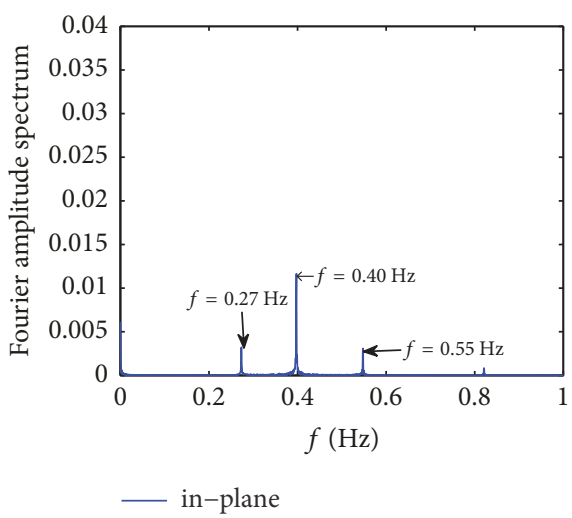

(c) Fourier spectrum of the in-plane mid-span displacement

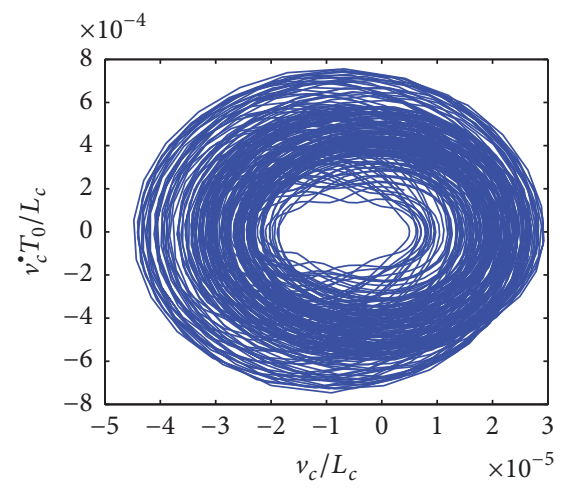

(e) In-plane mid-span phase trajectory



-. - dynamic tension

— initial tension

(g) Dynamic tension time history

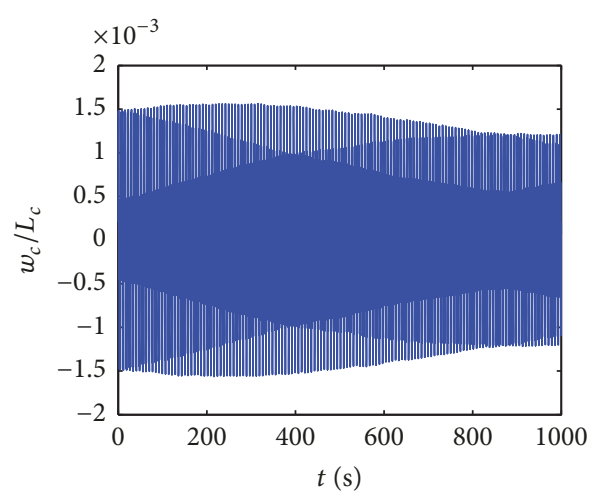

(b) Out-of-plane mid-span displacement

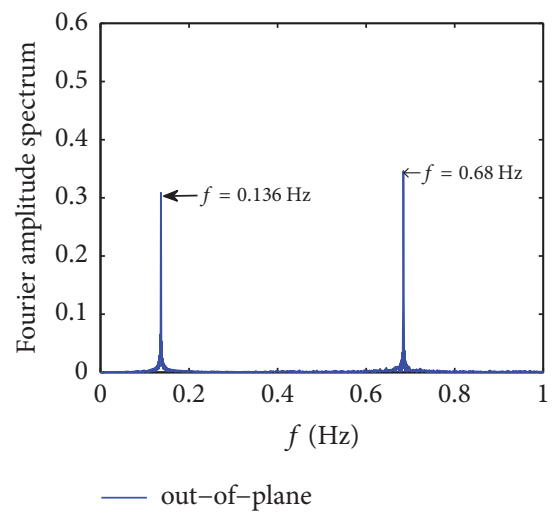

(d) Fourier spectrum of the out-of-plane midspan displacement

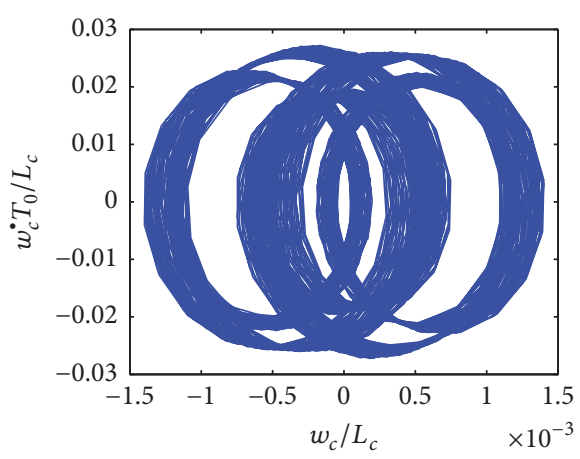

(f) Out-of-plane mid-span phase trajectory

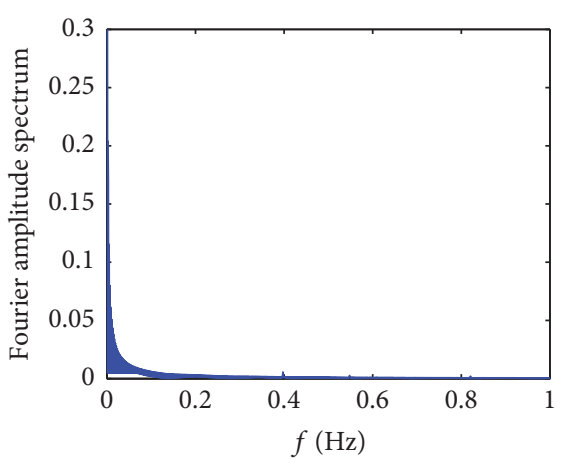

(h) Fourier spectrum of the dynamic tension

FIGURE 18: Nonresonance response of the transmission line when its damping $c_{c}$ is set to 0.001 . 


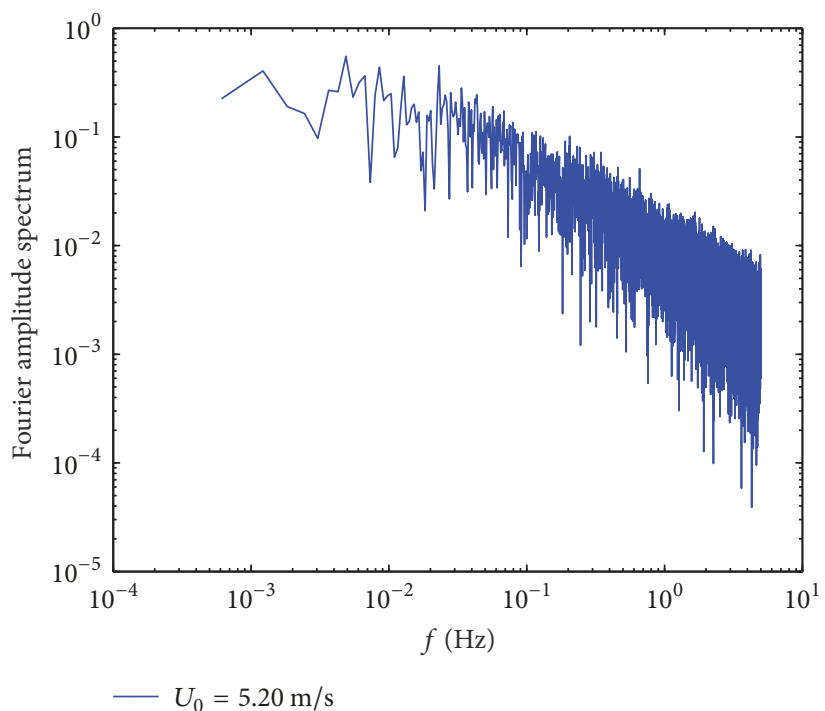

(a) Fourier spectrum of the wind speed $\left(U_{0}=5.2 \mathrm{~m} / \mathrm{s}\right)$

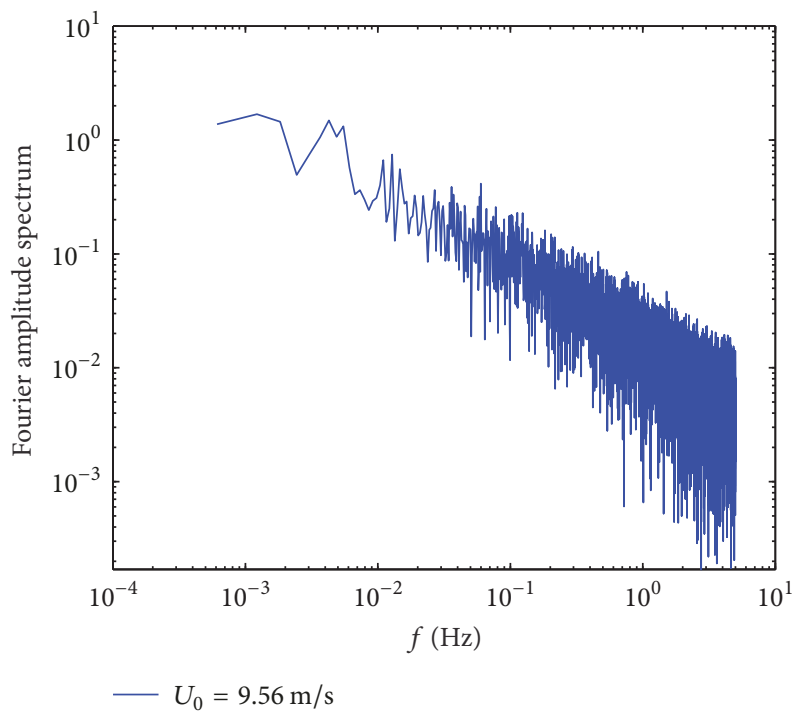

(b) Fourier spectrum of the wind speed $\left(U_{0}=9.56 \mathrm{~m} / \mathrm{s}\right)$

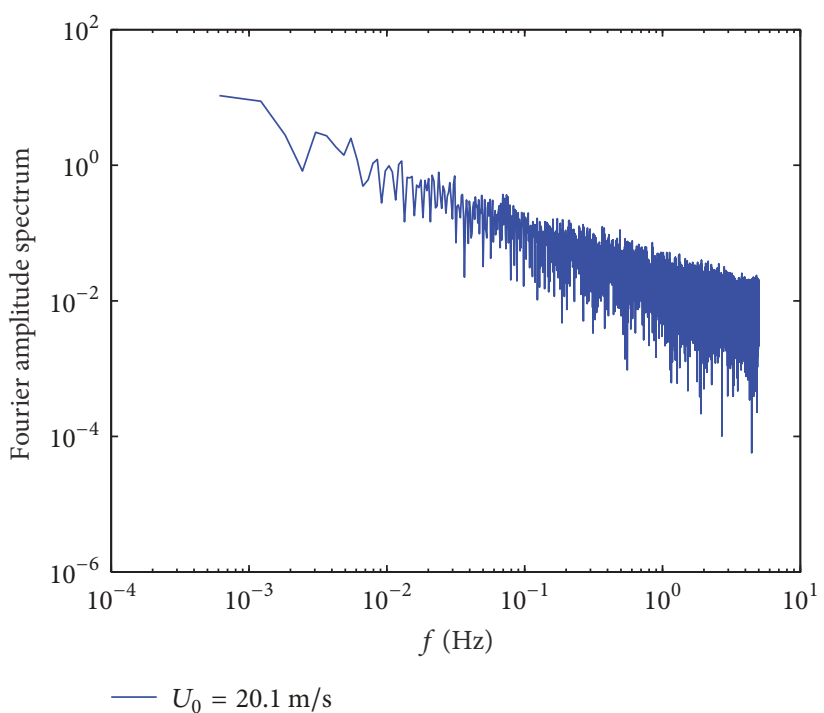

(c) Fourier spectrum of the wind speed $\left(U_{0}=20.1 \mathrm{~m} / \mathrm{s}\right)$

FIGURE 19: Fourier spectra of the wind speed.

the internal resonance, the natural characteristics of the inplane and out-of-plane vibration undergo relatively significant changes, and its energy is distributed in a relatively wide frequency band (Figures 20(e) and 20(f)). In addition, due to the in-plane vibration, there are significant jumps in the dynamic tension amplitude of the transmission line (Figures $20(\mathrm{~g})$ and $20(\mathrm{~h})$ ).

Figure 21 shows the simulation results obtained when the average wind speed $U_{0}$ is increased to $9.56 \mathrm{~m} / \mathrm{s}$. As demonstrated in Figure 21, the transmission line vibrates with a large amplitude; in addition, the out-of-plane mid-span vibration amplitude of the transmission line can reach half of its maximum sag, and its in-plane mid-span vibration amplitude can also reach half of its maximum out-of-plane vibration amplitude (Figure 21(a)); moreover, there are also jumps in the in-plane and out-of-plane vibration amplitudes of the transmission line. However, due to the inherent randomness of a wind load, the internal resonance of the transmission line when in wind-induced vibration is not as conspicuous as the internal resonance that occurs when the line is in free vibration or under a harmonic excitation. However, as demonstrated by the response frequency spectra, changes in the natural vibration characteristics of the system are more significant than those that occur in a linear system. Higher-order in-plane and out-of-plane modes of vibration of the transmission line can be excited by a wind load with a relatively high wind speed (Figures 21(e) and 21(f)); under this condition, the phase trajectory of the response has an oval shape, and there are jumps in the displacement amplitude (the outer and inner trajectories represent lower-order and higher-order modes of 


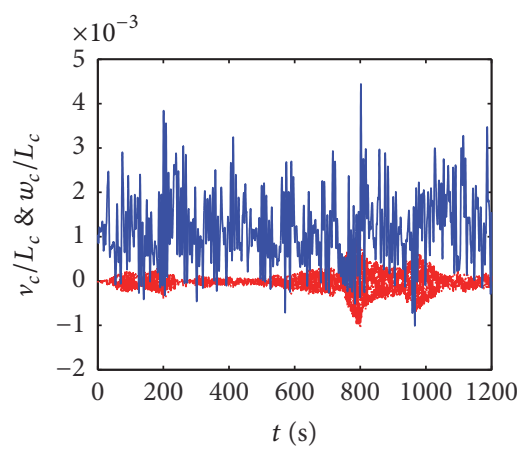

-.- $1 / 2 L_{c}$ in-plane out-of-plane

- $u_{0}=5.20 \mathrm{~m} / \mathrm{s}$

(a) Dimensionless mid-span displacement

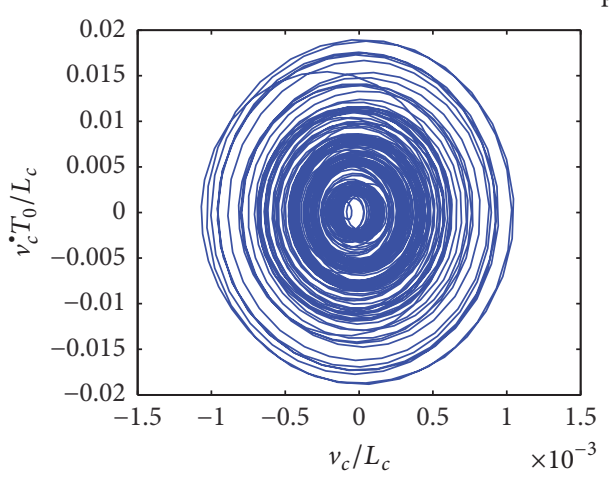

(c) In-plane mid-span phase trajectory

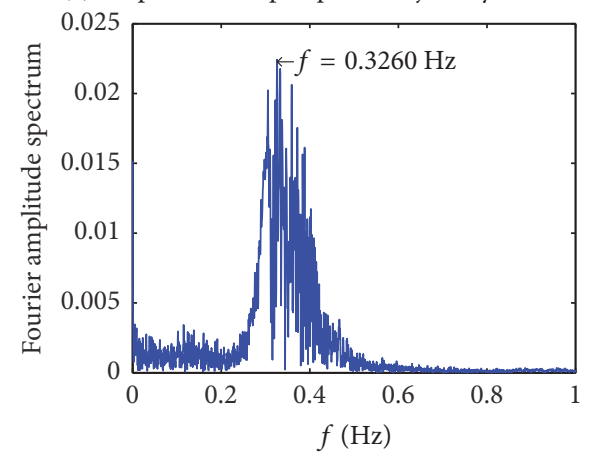

— in-plane

(e) Fourier spectrum of the in-plane mid-span displacement

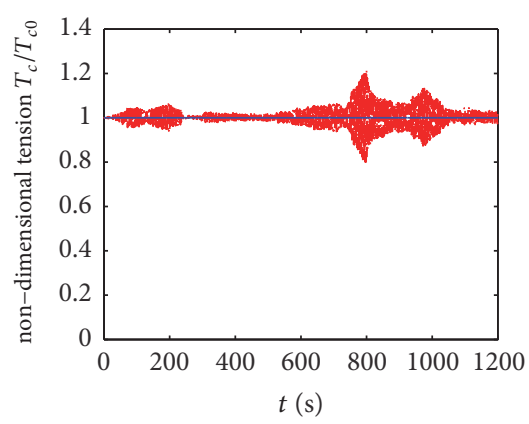

dynamic tension

— initial tension

(g) Dynamic tension time history

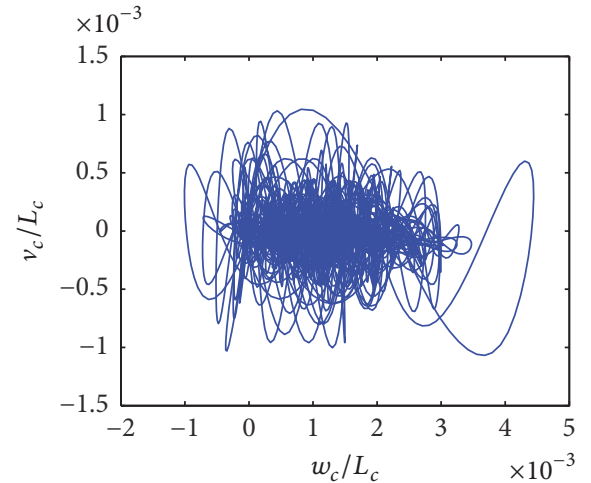

(b) Relationship between the out-of-plane and inplane mid-span displacements

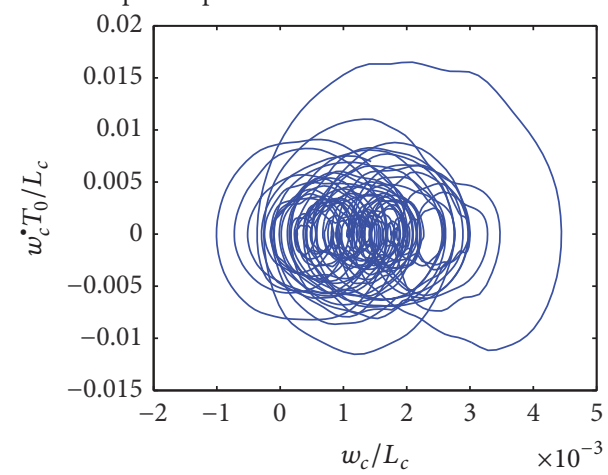

(d) Out-of-plane mid-span phase trajectory

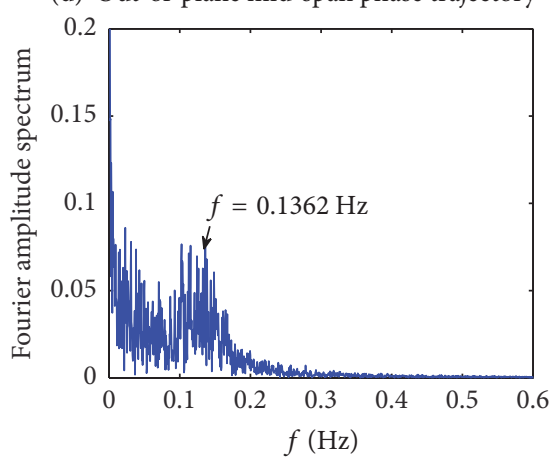

_ out-of-plane

(f) Fourier spectrum of the out-of-plane midspan displacement

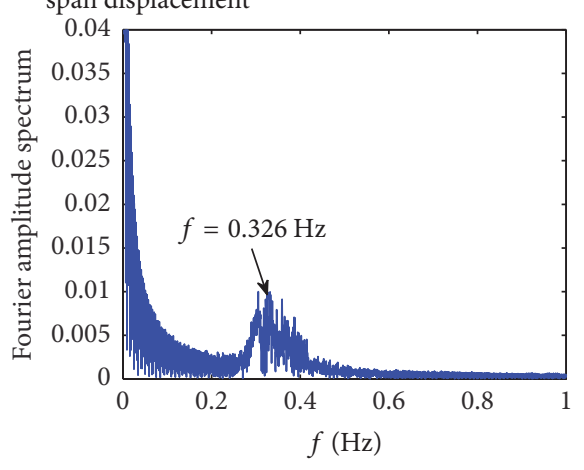

(h) Fourier spectrum of the dynamic tension

FIGURE 20: Wind-induced vibration response of the transmission line when $U_{0}=5.2 \mathrm{~m} / \mathrm{s}$. 


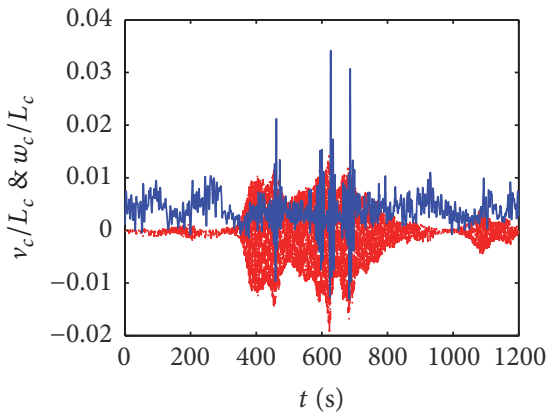

-.- $1 / 2 L_{c}$ in-plane

out-of-plane
$u_{0}=9.56 \mathrm{~m} / \mathrm{s}$

(a) Dimensionless mid-span displacement

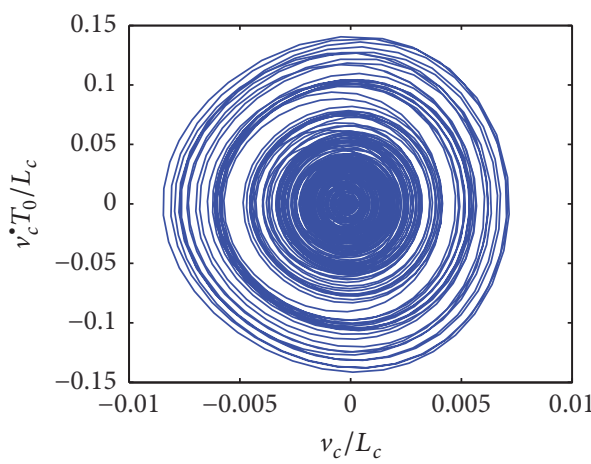

(c) In-plane mid-span phase trajectory

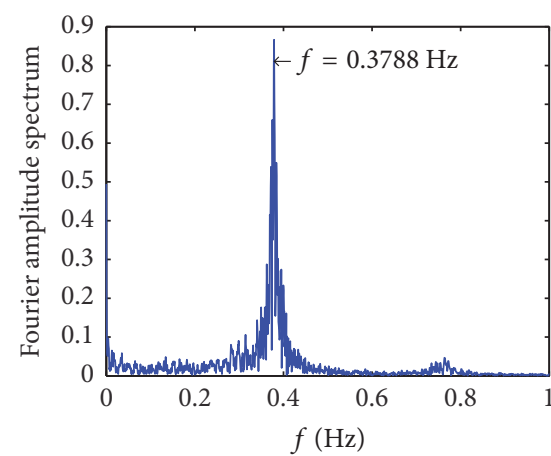

— in-plane

(e) Fourier spectrum of the in-plane mid-span displacement

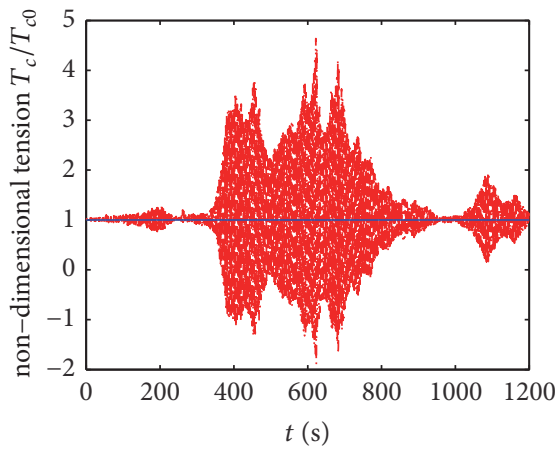

-. - dynamic tension

— initial tension

(g) Dynamic tension time history

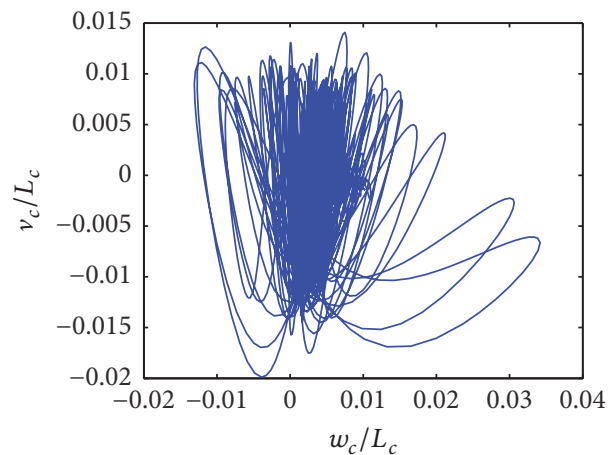

(b) Relationship between the out-of-plane and inplane mid-span displacements

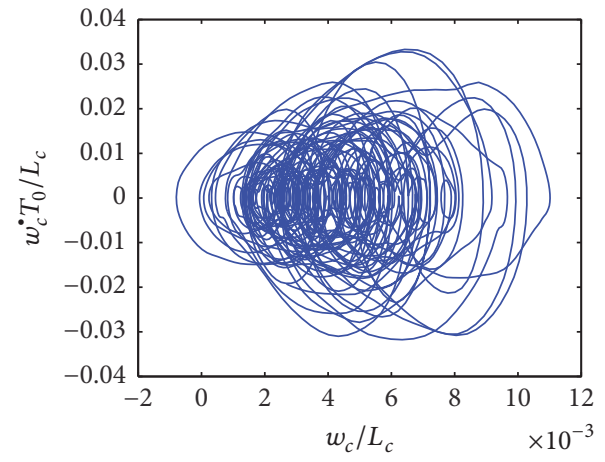

(d) Out-of-plane mid-span phase trajectory

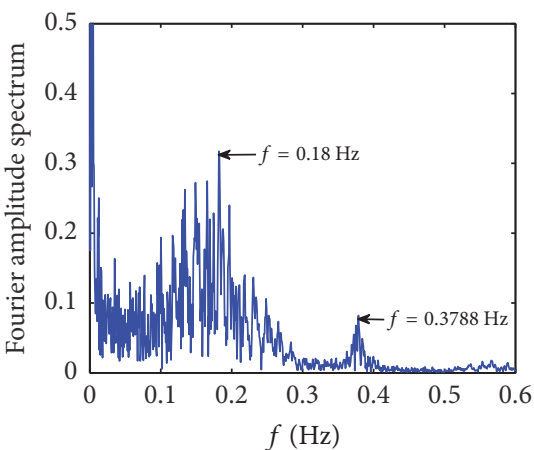

_ out-of-plane

(f) Fourier spectrum of the out-of-plane midspan displacement

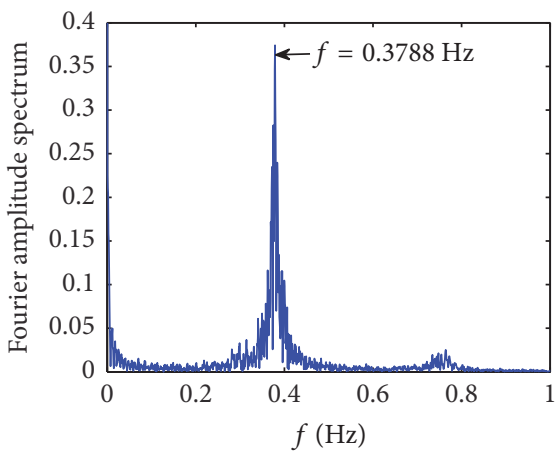

(h) Fourier spectrum of the dynamic tension

FIGURE 21: Wind-induced vibration response of the transmission line when $U_{0}=9.56 \mathrm{~m} / \mathrm{s}$. 


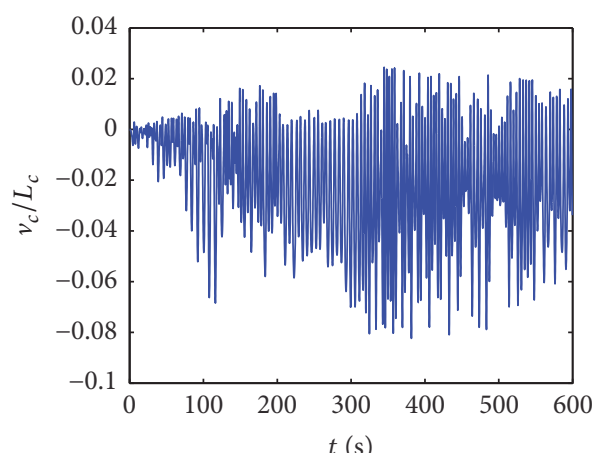

(a) Dimensionless in-plane mid-span displacement

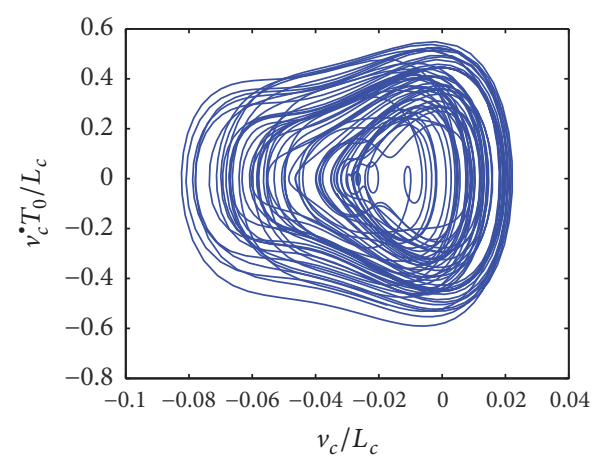

(c) In-plane mid-span phase-plane response

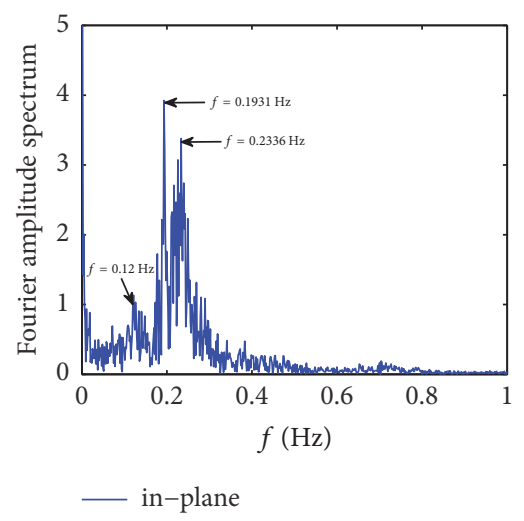

(e) Fourier spectrum of the in-plane midspan displacement response

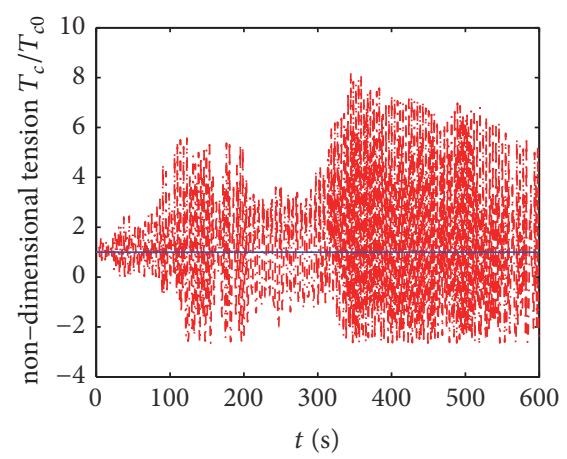

-.- dynamic tension

_ initial tension

(g) Dynamic tension time history

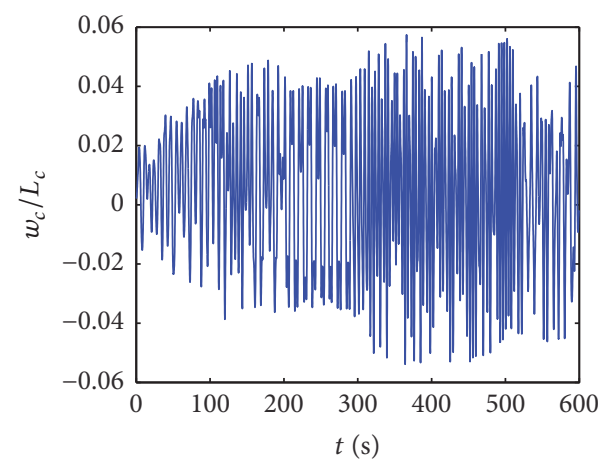

(b) Dimensionless out-of-plane mid-span displacement

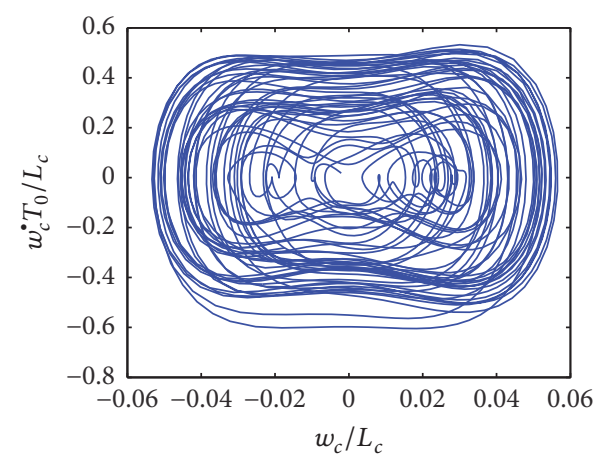

(d) Out-of-plane mid-span phase-plane response

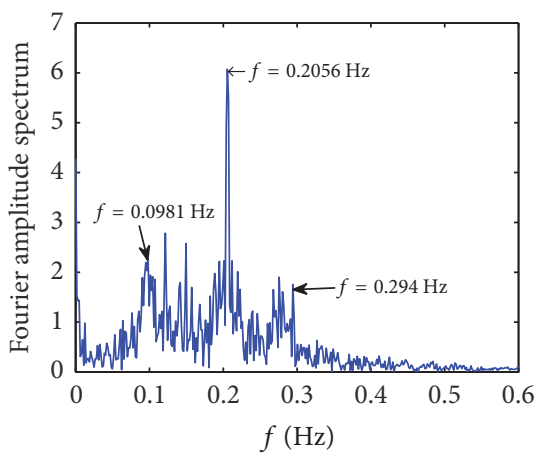

- out-of-plane

(f) Fourier spectrum of the out-of-plane midspan displacement response

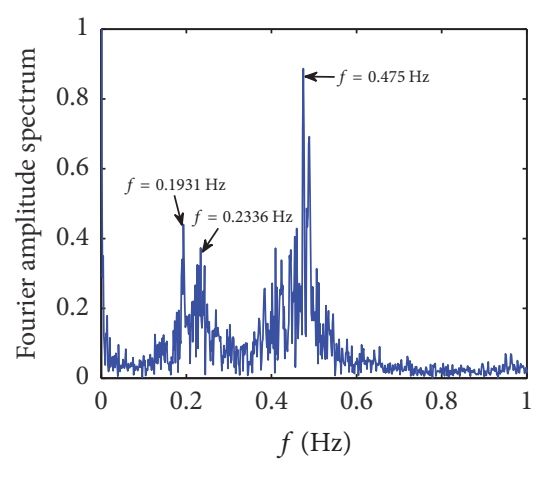

(h) Fourier spectrum of the dynamic tension

FIGURE 22: Wind-induced vibration response of the transmission line when $U_{0}=20.1 \mathrm{~m} / \mathrm{s}$. 
vibration, resp.) (Figures 21(c) and 21(d)). A comparison of Figures 20 and 21 shows that as the wind speed increases, the dynamic tension in the transmission line increases. Because the dynamic tension is mainly affected by the in-plane vibration, the jumps in the dynamic tension amplitude are quite noticeable. If the external wind excitation lasts for a sufficiently long period of time, the dynamic tension in the transmission line can even surpass its breaking load limit at some moments during the vibration process (Figures $21(\mathrm{~g})$ and $21(\mathrm{~h})$ ), resulting in damage to the transmission line. This should warrant attention in practical engineering.

Figure 22 shows the simulation results obtained when the average wind speed $U_{0}$ is increased to $20.1 \mathrm{~m} / \mathrm{s}$. As demonstrated in Figure 22, the vibration amplitude of the transmission line further increases under a strong wind; in addition, the in-plane vibration amplitude of the transmission line is even greater than its out-of-plane vibration amplitude, and the out-of-plane and in-plane vibration amplitudes of the transmission line are of the same order of magnitude as its maximum arc sag (Figures 22(a) and 22(b)) and can even surpass its maximum arc sag, which will result in damage to the transmission line under practical conditions. As demonstrated in the in-plane and out-of-plane displacement response time histories and phase-plane response (Figures 22(c) and 22(d)), the wind-induced vibration of the transmission line exhibits significant nonlinear vibration characteristics; in addition, the out-of-plane vibration is also coupled to the in-plane vibration, and the vibration energy of the structure transfers from the out-of-plane modes that are under the wind load to the in-plane modes.

A comparison of Figures 22(e) and 22(f) shows that the energy-containing range of the out-of-plane mid-span displacement response of the transmission line is $0.09-0.294 \mathrm{~Hz}$ and the energy-containing range of its in-plane mid-span displacement response is $0.12-0.25 \mathrm{~Hz}$, whereas the main energy-containing range of the wind load is $0.01-1 \mathrm{~Hz}$. Thus, under a strong wind, the wind-induced vibration of the transmission line modulates not only the line's vibration amplitude but also its vibration frequency and can cause the transmission line to undergo high-frequency vibration; in addition, the dynamic tension in the transmission line increases significantly and can even reach or surpass its breaking load in a short time, which is very disadvantageous to the transmission line under practical conditions. A comparison of Figures 14 and 22 shows that the motion of the transmission line under a strong wind is similar to that of a primary resonance under harmonic excitation; that is, the amplitude of vibration is very large, and the in-plane vibration is strongly coupled to its out-of-plane vibration.

\section{Conclusions}

In this study, 3D nonlinear vibration equations for transmission lines that consider geometric nonlinearity are established. Based on the boundary conditions and the assumptions used for modeling, the 3D equations are simplified to $2 \mathrm{D}$ equations. Galerkin's modal truncation method is employed to simplify the continuous partial differential equations to discrete partial differential equations that are analyzed using the method of multiple scales and solved by a higher-order Runge-Kutta method. On this basis, the nonlinear dynamic characteristics of transmission lines when in free and forced vibration, respectively, are analyzed. The main conclusions drawn from this study are summarized as follows:

(1) A transmission line has inherent nonlinear vibration characteristics. When in vibration, the in-plane motion of a transmission line is coupled to its out-of-plane motion, and linear, quadratic nonlinear, and cubic nonlinear modes of internal resonance can easily occur.

(2) When a transmission line vibrates freely, nonlinear internal resonance can occur even when the initial outof-plane energy of the transmission line is relatively low; however, under this condition, energy transfer and mode coupling phenomena are not noticeable. As its initial outof-plane energy increases, the internal resonance of a transmission line becomes prominent and the coupling of its inplane and out-of-plane vibration becomes stronger, resulting in a transfer of out-of-plane vibration energy to the inplane direction and an increase in the in-plane vibration amplitude and the dynamic tension in the transmission line. Due to the transmission line's mode coupling and nonlinear internal resonance, the out-of-plane and in-plane vibration frequencies of the line differ relatively significantly from the natural vibration frequencies of a linear system.

(3) Under a harmonic excitation, a transmission line undergoes forced resonance. Under the combined action of internal and primary resonance, the in-plane vibration of the transmission line is coupled to its out-of-plane vibration and the vibration energy transfers from the out-of-plane direction to the in-plane direction that is not directly under the excitation, resulting in a significant increase in the dynamic tension and the displacement amplitude of the transmission line. In addition, as the external excitation amplitude increases, the forced resonance effects become more significant, and the forced resonance has a modulating effect on the vibration frequency of the transmission line. As a result of the forced resonance, the in-plane and out-of-plane vibration energy of the transmission line is distributed over a relatively wide range.

(4) When a transmission line is in wind-induced vibration, as the wind speed increases, the nonlinear internal resonance of the transmission line increases; the frequencymodulating effect of its nonlinear internal resonance also increases, which may cause the transmission line to undergo high-frequency vibration. Because the in-plane vibration amplitude of a transmission line and its dynamic tension increase significantly as the wind speed increases, a noticeable amplitude jumping phenomenon occurs; consequently, when in vibration for a long period of time, the dynamic tension amplitude of the transmission line may exceed the breaking load limit, resulting in the breaking of the transmission line.

(5) Regardless of whether a transmission line is in free or forced vibration, increasing its damping can effectively consume its vibration energy, thereby allowing the vibration amplitude of the transmission line to reach a stable state within a short period of time. However, increasing the damping cannot prevent the occurrence of nonlinear internal resonance in a transmission line. When a transmission line 
is in forced vibration and the external excitation has a relatively large amplitude, even if the damping of the transmission line is increased to a relatively large value, the transmission line can still maintain its nonlinear vibration of coupled modes with a large amplitude due to the nonlinear internal resonance; in addition, the vibration energy also constantly transfers between the coupled modes and does not decrease significantly. When a transmission line is in vibration for an extended period of time, this response saturation phenomenon can still cause the dynamic tension in the transmission line to surpass the design-breaking load, thereby causing damage to the transmission line.

\section{Conflicts of Interest}

The authors declare that there are no conflicts of interest regarding the publication of this paper.

\section{Acknowledgments}

This research has been funded by the National Natural Science Foundation of China (Grants nos. 51578512 and 51108425), Outstanding Young Talent Research Fund of Zhengzhou University (Grant no. 1521322004), and Foundation for University Young Key Teacher by Henan Province (Grant no. 2015GGJS-151).

\section{References}

[1] H. M. Irvine, Cable Structure, MIT Press, 1981.

[2] H. M. Irvine and J. H. Griffin, "On the dynamic response of a suspended cable," Earthquake Engineering \& Structural Dynamics, vol. 4, no. 4, pp. 389-402, 1976.

[3] H. Yamaguchi and M. Ito, "Linear theory of free vibrations of an inclined cable in three dimensions," Proceedings of the Japan Society of Civil Engineers, vol. 1979, no. 286, pp. 29-36, 1979.

[4] H. Yamaguchi, T. Miyata, and M. Ito, "Time response analysis of a cable under harmonic excitation," Proceedings of the Japan Society of Civil Engineers, vol. 1981, no. 308, pp. 424-427, 1981.

[5] N. C. Perkins and C. D. Mote Jr., "Three-dimensional vibration of travelling elastic cables," Journal of Sound and Vibration, vol. 114, no. 2, pp. 325-340, 1987.

[6] G. Visweswara Rao and R. N. Iyengar, "Internal resonance and non-linear response of a cable under periodic excitation," Journal of Sound and Vibration, vol. 149, no. 1, pp. 25-41, 1991.

[7] F. Benedettini, G. Rega, and R. Alaggio, "Non-linear oscillations of a four-degree-of-freedom model of a suspended cable under multiple internal resonance conditions," Journal of Sound and Vibration, vol. 182, no. 5, pp. 775-798, 1995.

[8] M. Aufaure, "Three-node cable element ensuring the continuity of the horizontal tension; A clamp-cable element," Computers \& Structures, vol. 74, no. 2, pp. 243-251, 2000.

[9] W. K. Chang and R. A. Ibrahim, "Multiple Internal Resonance in Suspended Cables under Random In-Plane Loading," Nonlinear Dynamics, vol. 12, no. 3, pp. 275-303, 1997.

[10] A. S. K. Kwan, "A new approach to geometric nonlinearity of cable structures," Computers \& Structures, vol. 67, no. 4, pp. 243252, 1998.
[11] H. N. Arafat and A. H. Nayfeh, "Non-linear responses of suspended cables to primary resonance excitations," Journal of Sound and Vibration, vol. 266, no. 2, pp. 325-354, 2003.

[12] N. Srinil, G. Rega, and S. Chucheepsakul, “Three-dimensional non-linear coupling and dynamic tension in the large-amplitude free vibrations of arbitrarily sagged cables," Sound and Vibration, vol. 269, no. 3-5, 852 pages, 2004.

[13] N. Srinil, G. Rega, and S. Chucheepsakul, “Two-to-one resonant multi-modal dynamics of horizontal/inclined cables. I. Theoretical formulation and model validation," Nonlinear Dynamics, vol. 48, no. 3, pp. 231-252, 2007.

[14] X. Xiao, J. Yang, J. Druez, and Y. Xu, "Nonlinear oscillations of a suspended cable," Zhendong Ceshi Yu Zhenduan/Journal of Vibration, Measurement and Diagnosis, vol. 23, no. 2, p. 110, 2003.

[15] D. Dempsey, "Winds wreak havoc on lines," Transmission Distribution World, vol. 48, no. 6, pp. 32-40, 1996.

[16] K. Wang and G. Tang, "Response analysis of nonlinear vibration of overhead power line under suspension chain state," Vibration and Shock, vol. 22, no. 2, 72 pages, 2003.

[17] N. Barbieri, O. H. de Souza Júnior, and R. Barbieri, "Dynamical analysis of transmission line cables. Part 1-Linear theory," Mechanical Systems and Signal Processing, vol. 18, no. 3, pp. 659669, 2004.

[18] R. Barbieri, N. Barbieri, and O. H. de Souza Júnior, "Dynamical analysis of transmission line cables. Part 3-Nonlinear theory," Mechanical Systems and Signal Processing, vol. 22, no. 4, pp. 9921007, 2008.

[19] A. Berlioz and C.-H. Lamarque, "A non-linear model for the dynamics of an inclined cable," Journal of Sound and Vibration, vol. 279, no. 3-5, pp. 619-639, 2005.

[20] S. W. Rienstra, "Nonlinear free vibrations of coupled spans of overhead transmission lines," Journal of Engineering Mathematics, vol. 53, no. 3-4, pp. 337-348, 2005.

[21] K. Tucker and A. Haldar, "Numerical model validation and sensitivity study of a transmission-line insulator failure using full-scale test data," IEEE Transactions on Power Delivery, vol. 22, no. 4, pp. 2439-2444, 2007.

[22] G. F. Zhao, Q. Xie, and J. Li, "Wind-induced nonlinear interactions in the out-of-plane and in-plane dynamics of transmission line conductors," in Proceedings of the in Proceedings of Second International Conference on Modelling and Simulation, pp. 8085, UK, 2009.

[23] L. Wang and B. Fang, "Simulations on transient characteristics of $500 \mathrm{kV}$ capacitor voltage transformer," Gaodianya Jishu/High Voltage Engineering, vol. 38, no. 9, pp. 2389-2396, 2012.

[24] X. Xie, X. Hu, J. Peng, and Z. Wang, "Refined modeling and free vibration of two-span suspended transmission lines," Acta Mechanica, vol. 228, no. 2, pp. 673-681, 2017.

[25] A. H. Nayfeh and D. T. Mook, Nonlinear Oscillations, John Wiley \& Sons, New York, NY, USA, 1979.

[26] B. Wen, Y. Li, P. Xu, and Q. Han, Engineering Nonlinear Vibration, Science Press, Beijing, China, 2007.

[27] G. Veronis, "A note on the method of multiple scales," Quarterly of Applied Mathematics, vol. 38, no. 3, pp. 363-368, 1980/81.

[28] H. Wang and H. Hu, "Remarks on the perturbation methods in solving the second-order delay differential equations," Nonlinear Dynamics, vol. 33, no. 4, pp. 379-398, 2003. 
[29] Hu. Huailei WangHaiyan, "A note on the method of multiple scales," Journal of Dynamics and Control, vol. 2, no. 3, pp. 10-13, 2004.

[30] M. Zhang, G. Zhao, L. Wang, and J. Li, "Wind-induced coupling vibration effects of high-voltage transmission tower-line systems," Shock and Vibration, vol. 2017, 34 pages, 2017. 


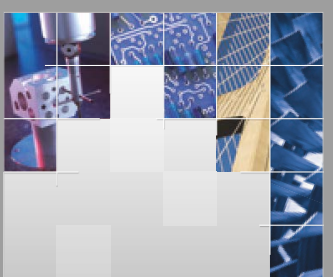

\section{Enfincering}
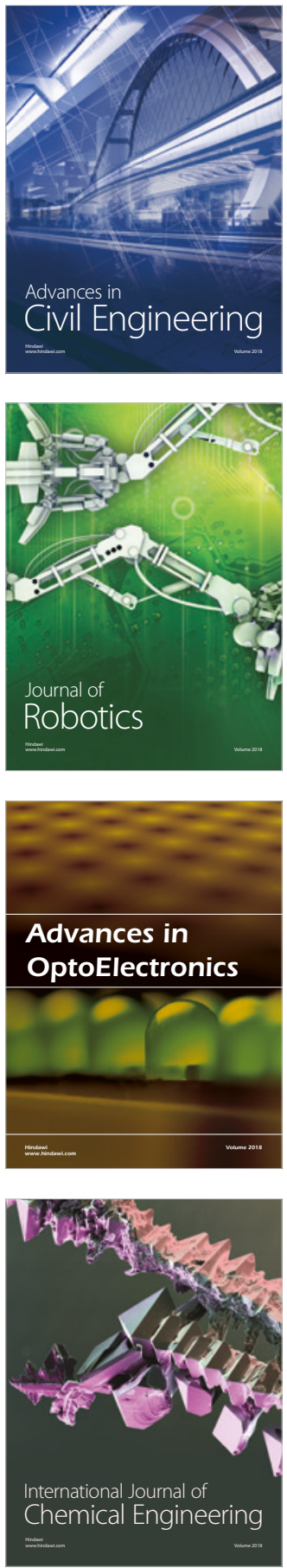

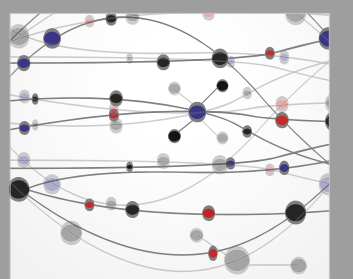

\section{Rotating \\ Machinery}

The Scientific World Journal

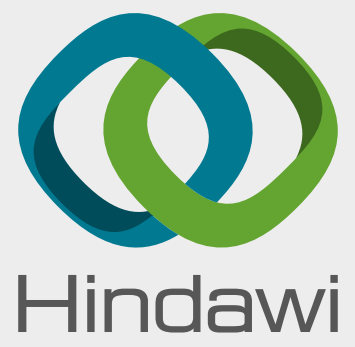

Submit your manuscripts at

www.hindawi.com
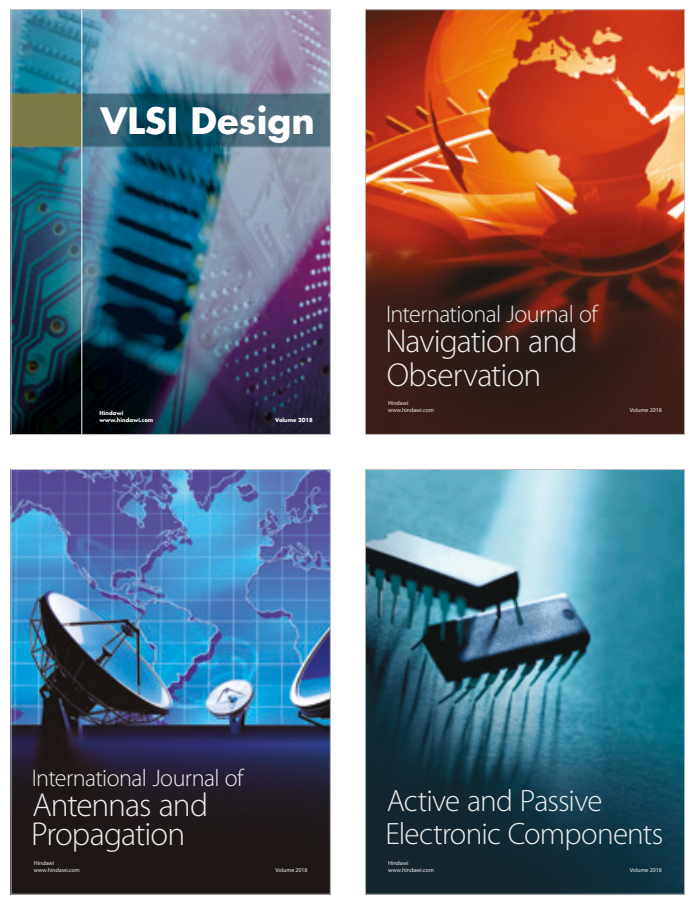
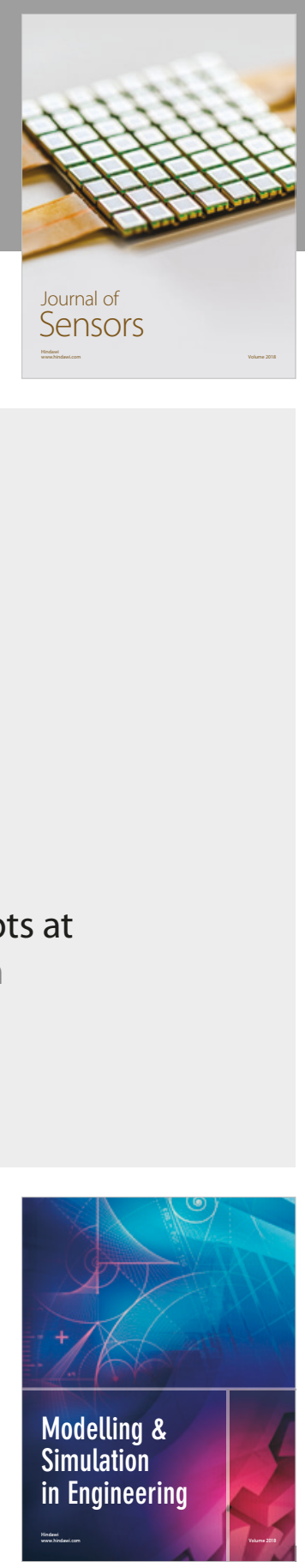

\section{Advances \\ Multimedia}
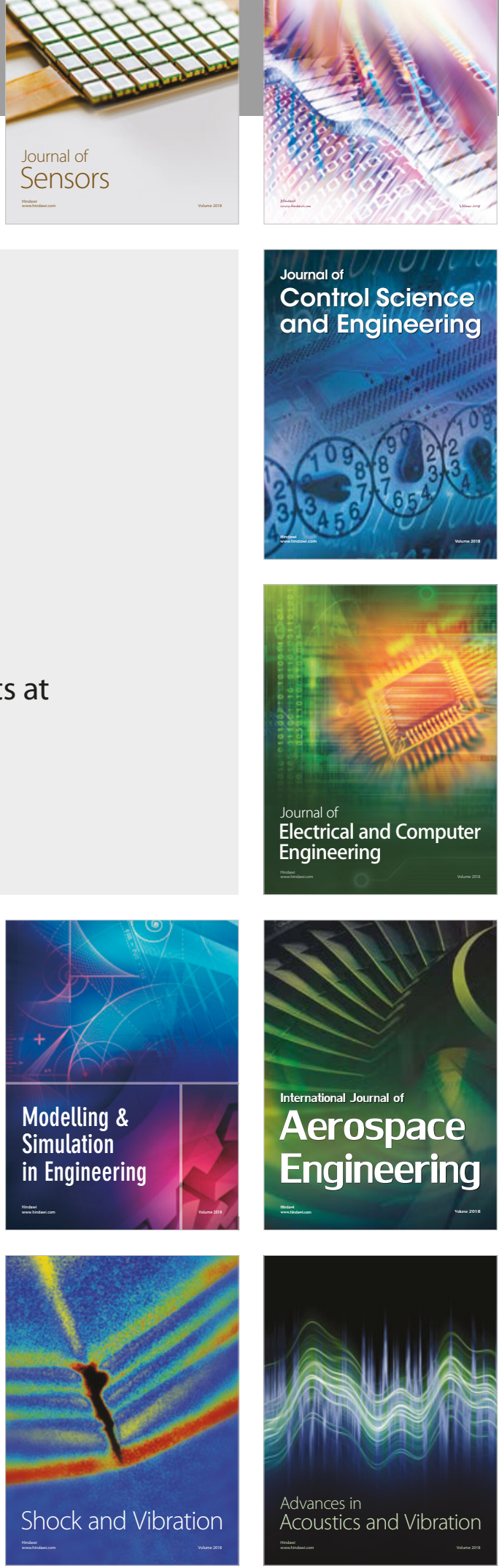